\title{
High phenotypic plasticity of Ganoderma sinense (Ganodermataceae, Polyporales) in China
}

\author{
Hapuarachchi $\mathrm{KK}^{3}, 4$, Karunarathna $\mathrm{SC}^{5}$, McKenzie $\mathrm{EHC}^{6}$, Wu $\mathrm{XL}^{7}$, \\ Kakumyan $\mathrm{P}^{4}$, Hyde $\mathrm{KD}^{2,3,4}$, Wen $\mathrm{TC}^{1,2 *}$
}

\author{
${ }^{1}$ State Key Laboratory Breeding Base of Green Pesticide and Agricultural Bioengineering, Key Laboratory of Green \\ Pesticide and Agricultural Bioengineering, Ministry of Education, Guizhou University, Guiyang 550025, China \\ ${ }^{2}$ The Engineering Research Center of Southwest Bio-Pharmaceutical Resource Ministry of Education, Guizhou \\ University, Guiyang 550025, Guizhou Province, China \\ ${ }^{3}$ Center of Excellence in Fungal Research, Mae Fah Luang University, Chiang Rai 57100, Thailand \\ ${ }^{4}$ School of Science, Mae Fah Luang University, Chiang Rai 57100, Thailand \\ ${ }^{5}$ Key Laboratory for Plant Diversity and Biogeography of East Asia, Kunming Institute of Botany, Chinese Academy of \\ Sciences, 132 Lanhei Road, Kunming 650201, China \\ ${ }^{6}$ Manaaki Whenua Landcare Research, Private Bag 92170, Auckland, New Zealand \\ ${ }^{7}$ Guizhou Academy of Sciences, Guiyang, 550009, Guizhou Province, China
}

Hapuarachchi KK, Karunarathna SC, McKenzie EHC, Wu XL, Kakumyan P, Hyde KD, Wen TC 2019 - High phenotypic plasticity of Ganoderma sinense (Ganodermataceae, Polyporales) in China. Asian Journal of Mycology 2(1), 1-47, Doi 10.5943/ajom/2/1/1

\begin{abstract}
Ganoderma is a typical polypore genus which has been used in traditional medicines for centuries and is increasingly being used in pharmaceutical industries worldwide. The genus has been extensively researched due to its beneficial medicinal properties and chemical constituents. However, the taxonomy of Ganoderma remains unclear, and has been plagued by confusion and misinterpretations. During an extensive survey of Ganoderma we were found $20 \mathrm{G}$. sinense specimens from China. The specimens were identified as $G$. sinense with the aid of molecular phylogenetic evidence. The studied specimens exhibit phenotypic plasticity due to extrinsic factors, even though they possess identical nucleotide sequences. Seven sequences derived from this study have a single nucleotide polymorphism located at position 158 in ITS1 region and all G. sinense collections clustered in the same species clade. The specimens are each described, illustrated with coloured photographs and compared to similar taxa. We provide a phylogenetic tree for Ganoderma based on ITS, SSU, LSU and RPB2 sequence data and the taxonomic status of $G$. sinense is discussed.
\end{abstract}

Key words - Environment factors - Morphological characteristics - Phylogeny - Taxonomy

\section{Introduction}

The genus Ganoderma was established by Karsten (1881) with G. lucidum (Curtis) P. Karst. as the type species. It is a diverse genus that occurs throughout the world. About 450 epithets have been listed (Index Fungorum, accessed 27 February 2019). Ganoderma species grow as facultative parasites that can also live as saprobes on decaying stumps or associated with roots of living and dead trees (Singh et al. 2007). They are not edible, since the basidiomes are always corky, tough and bitter in taste (Hapuarachchi et al. 2015). Two types of basidiomes are produced, laccate with a 
shiny upper surface, and non-laccate with a dull upper surface (Smith \& Sivasithamparam 2003, Pilotti et al. 2004). Ganoderma species have been broadly utilized as traditional medicines for millennia in Asia (Zhou et al. 2015, Hapuarachchi et al. 2018a) and, because of their highly prized medicinal value are widely researched (De Silva et al. 2012a, b, 2013, Hapuarachchi et al. 2016a, b, 2017, 2018c). Ganoderma species are economically important not only because of their medicinal properties, but also because of their phytopathogenicity (Dai et al. 2007, 2009).

Ganoderma is characterized by having ellipsoid to ovoid basidiospores, with a truncate apex and an endosporium with columnar ornamentations (Costa-Rezende et al. 2017). Ganoderma is morphologically the most complex polypore genus and characteristics such as shape, colour of the fruit body, host-specificity and geographical origin, are used to identify species (Szedlay 2002, Hapuarachchi et al 2018b). Species identification is complicated and has been widely debated (Moncalvo et al. 1995a, Wang et al. 2009, Cao et al. 2012, Yao et al. 2013, Richter et al. 2015, Zhou et al. 2015). Ganoderma species are genetically heterogeneous because of geographical differentiation and high genetic diversity (Miller et al. 1999, Pilotti et al. 2003). This high genetic diversity is associated with substantial morphological variation, even within species and hence, naming a species within this genus is often ambiguous (Hong et al. 2001, 2002). High phenotypic plasticity at the macroscopic level and the uniformity of microscopic characteristics, subjective interpretation of various features, lack of keys and accessible type specimens, and presence of single nucleotide polymorphism have resulted in numerous unnecessary species names (Seo \& Kirk 2000, Zhang et al. 2017). Hence, DNA sequence data play a crucial role in identifying species (Cao et al. 2012, Yang \& Feng 2013, Pawlik et al. 2015). Phylogenetic analyses have proved that extensive convergence or parallelism of morphological characteristics has occurred during evolution of Ganoderma (Hong \& Jung 2004, Moncalvo 2005). The specific interhybridization and genetic background of Ganoderma remains relatively unclear and the genetic distance between Ganoderma species has not been evaluated (Zheng et al. 2009). Ganoderma was first reported from China by Teng (1934) and later, many researchers extensively studied this genus and about 100 species were recorded (Zhao \& Zhang 2000, Wu \& Dai 2005, Cao et al. 2012). Ganoderma sinense was originally described from China by Zhao et al. (1979) and is characterized by slightly longitudinally crested basidiospores and a uniformly brown to dark brown context. This species has been morphologically confused with G. lucidum (Curtis) P. Karst., G. japonicum (Fr.) Sawada (= G. dimidiatum (Thunb.) V. Papp) and G. orbiforme (Fr.) Ryvarden but, later researchers have distinguished them from G. sinense (Pegler \& Yao 1996, Wang et al. 2014).

Phenotypic plasticity is the development of different phenotypes from a single genotype, depending on the environment (Aubin-Horth \& Renn 2009). It is an extensive feature of life observed in various traits and is often contended to be the result of natural selection. Phenotypic plasticity includes an ecological and an evolutionary perspective, however, recent development in large-scale gene expression technology makes it feasible to study plasticity from a molecular perspective (Aubin-Horth \& Renn 2009). This phenomenon has been observed commonly in ascomycetes, due to the influence of environmental or cultural factors, and could easily result in misidentification as new taxa based on minor and statistically insignificant variation of morphological characteristics (Jeewon \& Hyde 2016). However, phenotypic plasticity in macroscopic fungi has been poorly studied and only general aspects have been described (RamírezLópez et al. 2013).

The development of basidiomes is influenced by the interaction of both intrinsic (genetic and physiological) and extrinsic (environmental) factors (Moore-Landecker 1996, Moore et al. 2008, 2011). In basidiomycetes, the development of basidiomes of Coprinus sp., Panus sp., Morchella sp., Pleurotus sp. and Typhula sp. is affected by environmental factors including the availability of nutrients, temperature, humidity, light, and pH (Morimoto \& Oda 1973, Bujakiewicz 1992, Kost 1992, Boulianne et al. 2000, Straatsma et al. 2001, Salerni et al. 2002, Kawakami et al. 2004, Singh et al. 2004, Ramírez-López et al. 2013). In general, the taxonomy of Ganoderma is considered uncertain due to the high phenotypic plasticity of the basidiomes at macroscopic level and uniformity of microscopic features (Sankaran et al. 2005). Gottlieb \& Wright (1999) have reported 
phenotypic plasticity in both micro- and macro-morphological characteristics of South American species of Ganoderma. Wu \& Dai (2005) mentioned that the morphology of Ganoderma species varies greatly due to influence of climate, nutrition, vegetation, and geographical environment and it is not in association with the genetic material of a particular species. Wu \& Dai (2005) described phenotypic plasticity of $G$. sinense in China based on different macro- and uniform micromorphological characteristics along with descriptions and colour photographs. In this study, we support the claims of $\mathrm{Wu} \&$ Dai (2005) based on strong molecular evidence together with morphological data. We describe $20 \mathrm{G}$. sinense specimens having different macro-morphological characteristics to each other based on combined ITS, LSU, SSU and RPB2 analyses, colour photographs and illustrations.

\section{Materials \& Methods}

\section{Sample collection}

Ganodermataceae were collected from Hainan during August 2014, and from Guizhou in September to October 2017. Collected materials were dealt with as described by Cao et al. (2012) and deposited at Guizhou University (GACP) and Mae Fah Luang University (MFLU) herbaria.

\section{Macroscopic and microscopic characterization}

Macro-morphological characteristics were described based on fresh materials, and on the photographs provided here. Colour codes (e.g. 6C8) are from Kornerup \& Wanscher (1978). Specimens were dried and placed separately in plastic bags. For micro-morphological observations, basidiomes were examined using a stereo dissecting microscope (Motic SMZ 168 series). Sections were cut with a razor blade and mounted in $5 \% \mathrm{KOH}$, and then observed, measured and illustrated using a Nikon ECLIPSE 80i compound microscope equipped with a camera (Canon 600D). Measurements were made using Tarosoft (R) Image Frame Work v. 0.9.7. At least 20 basidiospores were measured from each mature specimen, except for very scanty materials. The basidiospore size was measured both with and without the myxosporium, but only spore sizes with myxosporium were used for comparisons. Basidiospore dimensions are given as $(\mathrm{a}-) \mathrm{b}-c-\mathrm{d}(-\mathrm{e})$, where a represents the minimum, $\mathrm{b}$ (mean average-standard deviation), $c$ the average, $\mathrm{d}$ (mean average+standard deviation) and e the maximum. $Q$, the length/width ratio (L/W) of a spore in side view and $\boldsymbol{Q}_{\boldsymbol{m}}$ is the average, smallest and largest $\boldsymbol{Q}$ values given as $Q$. Pellis sections were taken from the mature pileus portion and mounted in Melzer's reagent for observation.

\section{DNA extraction, PCR and sequencing}

Dried samples of basidiomes were used to extract genomic DNA using an EZgene TM Fungal gDNA Kit (Biomiga, CA, USA) and following the manufacturer's instructions. DNA concentrations were estimated visually in agarose gel by comparing band intensity with a DNA ladder $1 \mathrm{~Kb}$ (Invitrogen Biotech). Reaction mixtures $(50 \mu \mathrm{l})$ contained $2 \mu 1$ template DNA (ca. 10 ng), $19 \mu \mathrm{l}$ distilled water, and $2 \mu \mathrm{l}(10 \mu \mathrm{M})$ of each primer and $25 \mu 12 \mathrm{x}$ BenchTopTM Taq Master Mix (Biomigas). Amplification conditions were 40 cycles of $95{ }^{\circ} \mathrm{C}$ for $30 \mathrm{~s}, 59{ }^{\circ} \mathrm{C}$ for $30 \mathrm{~s}$ and 72 ${ }^{\circ} \mathrm{C}$ for $1 \mathrm{~min}$, followed by a final extension at $72{ }^{\circ} \mathrm{C}$ for $10 \mathrm{~min}$ for all DNA fragments. The ITS rDNA regions were amplified using the universal primer pair ITS4 and ITS5 and the 18S and 28S rDNA genes were amplified using the universal primer pair NS1 and NS4 and primer pair LROR and LR5, respectively (Vilgalys \& Hester 1990, White et al. 1990, Rehner \& Samuels 1994). Protein coding gene, RNA polymerase II gene (RPB2) was amplified using primer pair fRPB26f/7CR (Liu et al. 1999). Amplified PCR products were verified by 1\% agarose gel electrophoresis stained with ethidium bromide in $1 \mathrm{x}$ TBE. The PCR products were sequenced by SinoGenoMax Co. (Beijing). 


\section{Sequence alignment and phylogenetic analysis}

Other sequences used in the analyses (Table 1) were retrieved from GenBank based on ITS BLAST searches (Benson et al. 2017) and recently published data. Sequences that had possibly been contaminated were unnamed species (such as those with aff. in the species name) were discarded, ambiguous regions were excluded and gaps were treated as missing data in the analyses (Nilsson et al. 2012). Seventy-four nucleotide sequences representing 22 species of Ganodermataceae from Africa, Asia, America and Europe were retrieved and all sequences were aligned with MAFFT v. 7 (http://mafft.cbrc.jp/alignment/server/index.html; Katoh \& Standley 2013). The resulting alignment was improved manually when necessary using BioEdit v. 7.0.5.2 (Hall 1999). Maximum likelihood (ML) analyses were performed using RAxML-HPC2 (Stamatakis 2014) on the CIPRES Science Gateway V. 3.3 (Miller \& Blair 2009), with default settings, except that the number of bootstrap replicates was set to 1,000. Maximum parsimony (MP) analysis was performed with PAUP version 4.0b10 (Swofford 2002) using a heuristic search and TBR (tree bisection-reconnection) swapping for 1000 random replicates. Gaps were set as "missing" data and the characters were specified as unordered and equally weighted for bootstrap analysis (Hillis \& Bull 1993) performed with 1000 replications with simple addition sequences to obtain estimates of reliability for nodes. For Bayesian analysis (BY), the GTR+I+G model of nucleotide evolution was selected with the help of MrModeltest 2.2 (Nylander 2004) as the best-fit model and posterior probabilities (PP) (Rannala \& Yang 1996) were determined by Markov Chain Monte Carlo sampling (BMCMC) using MrBayes v3.1.2 (Ronquist et al. 2012). BY analyses were conducted with six simultaneous Markov chains and trees were summarized every 100th generation. The analyses were stopped after 5,000,000 generations when the average standard deviation of split frequencies was below 0.01. The convergence of the runs was checked using TRACER v1.6 (Rambaut et al. 2013). The first 25\% of the resulting trees were discarded as burn-in, and PP were calculated from the remaining sampled trees. In both ML and BY analyses, Coriolopsis trogii was selected as the outgroup. ML, MP bootstrap values and BY posterior probabilities greater than or equal to $70 \%$ and 0.95 , respectively, were considered as significant support. The phylogenetic tree was visualized with FigTree version 1.4.0 (Rambaut 2012, available at http://tree.bio.ed.ac.uk/software/figtree/).

Table 1 Sequences used in the phylogenetic analysis

\begin{tabular}{|c|c|c|c|c|c|c|c|}
\hline Species & $\begin{array}{l}\text { Voucher } \\
\text { /strain }\end{array}$ & Origin & ITS & LSU & RPB2 & SSU & Reference \\
\hline $\begin{array}{l}\text { G. adspersum } \\
\text { (Schulzer) Donk }\end{array}$ & $\begin{array}{l}\text { SFC201410012 } \\
2\end{array}$ & Korea & $\begin{array}{l}\text { KY3642 } \\
52\end{array}$ & - & $\begin{array}{l}\text { KY3932 } \\
71\end{array}$ & - & $\begin{array}{l}\text { Jargalmaa et } \\
\text { al. } 2017\end{array}$ \\
\hline G. adspersum & $\begin{array}{l}\text { SFC201410011 } \\
6\end{array}$ & Korea & $\begin{array}{l}\text { KY3642 } \\
51\end{array}$ & - & $\begin{array}{l}\text { KY3932 } \\
70\end{array}$ & - & $\begin{array}{l}\text { Jargalmaa et } \\
\text { al. } 2017\end{array}$ \\
\hline $\begin{array}{l}\text { G. angustisporum } \\
\text { J.H. Xing, B.K. } \\
\text { Cui \& Y.C. Dai }\end{array}$ & $\begin{array}{l}\text { Cui } 13817 \\
\text { (holotype) }\end{array}$ & $\begin{array}{l}\text { Fujian, } \\
\text { China }\end{array}$ & $\begin{array}{l}\text { MG2791 } \\
70\end{array}$ & - & $\begin{array}{l}\text { MG3675 } \\
07\end{array}$ & - & $\begin{array}{l}\text { Xing et al. } \\
2018\end{array}$ \\
\hline G. angustisporum & Cui 14578 & $\begin{array}{l}\text { Guangdong, } \\
\text { China }\end{array}$ & $\begin{array}{l}\text { MG2791 } \\
71\end{array}$ & - & - & - & $\begin{array}{l}\text { Xing et al. } \\
2018\end{array}$ \\
\hline $\begin{array}{l}\text { G. applanatum } \\
\text { (Pers.) Pat. }\end{array}$ & $\begin{array}{l}\text { SFC201509300 } \\
2\end{array}$ & Korea & $\begin{array}{l}\text { KY3642 } \\
58\end{array}$ & - & $\begin{array}{l}\text { KY3932 } \\
74\end{array}$ & - & $\begin{array}{l}\text { Jargalmaa et } \\
\text { al. } 2017\end{array}$ \\
\hline G. applanatum & Dai8924 & China & $\begin{array}{l}\text { KU2199 } \\
87\end{array}$ & & - & - & $\begin{array}{l}\text { Song et al. } \\
2016\end{array}$ \\
\hline $\begin{array}{l}\text { G. aridicola J.H. } \\
\text { Xing \& B.K. Cui) }\end{array}$ & $\begin{array}{l}\text { Dai12588 } \\
\text { (holotype) }\end{array}$ & $\begin{array}{l}\text { Durban, } \\
\text { South } \\
\text { Africa }\end{array}$ & $\begin{array}{l}\text { KU5724 } \\
91\end{array}$ & - & - & - & $\begin{array}{l}\text { Xing et al. } \\
2016\end{array}$ \\
\hline $\begin{array}{l}\text { G. australe (Fr.) } \\
\text { Pat. }\end{array}$ & $\mathrm{K}(\mathrm{M}) 120828$ & UK & $\begin{array}{l}\text { AY } 8841 \\
83\end{array}$ & - & - & - & $\begin{array}{l}\text { Arulpandi \& } \\
\text { Kalaichelvan } \\
2013\end{array}$ \\
\hline G. australe & GDGM25344 & China & $\begin{array}{l}\text { JX19519 } \\
8\end{array}$ & - & - & - & GenBank \\
\hline
\end{tabular}


Table 1 Continued.

\begin{tabular}{|c|c|c|c|c|c|c|c|}
\hline Species & $\begin{array}{l}\text { Voucher } \\
\text { /strain }\end{array}$ & Origin & ITS & LSU & RPB2 & SSU & Reference \\
\hline $\begin{array}{l}\text { G. } \\
\text { austroafricanum } \\
\text { M.P.A. Coetzee, } \\
\text { M.J. Wingf., } \\
\text { Marinc. \& } \\
\text { Blanchette }\end{array}$ & $\begin{array}{l}\text { CMW41454 } \\
\text { (holotype) }\end{array}$ & $\begin{array}{l}\text { South } \\
\text { Africa }\end{array}$ & $\begin{array}{l}\text { KM5073 } \\
24\end{array}$ & $\begin{array}{l}\text { KM50 } \\
7325\end{array}$ & - & - & $\begin{array}{l}\text { Coetzee et } \\
\text { al. } 2015\end{array}$ \\
\hline $\begin{array}{l}\text { G. casuarinicola } \\
\text { J.H. Xing, B.K. } \\
\text { Cui \& Y.C. Dai }\end{array}$ & $\begin{array}{l}\text { Dai } 16336 \\
\text { (holotype) }\end{array}$ & $\begin{array}{l}\text { Guangdong, } \\
\text { China }\end{array}$ & $\begin{array}{l}\text { MG2791 } \\
73\end{array}$ & $\begin{array}{l}\text { MG36 } \\
7508\end{array}$ & $\begin{array}{l}\text { MG2791 } \\
73\end{array}$ & & $\begin{array}{l}\text { Xing et al. } \\
2016\end{array}$ \\
\hline G. casuarinicola & Dai 16338 & $\begin{array}{l}\text { Guangdong, } \\
\text { China }\end{array}$ & $\begin{array}{l}\text { MG2791 } \\
75\end{array}$ & - & $\begin{array}{l}\text { MG3675 } \\
10\end{array}$ & - & $\begin{array}{l}\text { Xing et al. } \\
2016\end{array}$ \\
\hline $\begin{array}{l}\text { G. destructans } \\
\text { M.P.A. Coetzee, } \\
\text { Marinc. \& M.J. } \\
\text { Wingf. }\end{array}$ & $\begin{array}{l}\text { CMW43670 } \\
\text { (holotype) }\end{array}$ & $\begin{array}{l}\text { South } \\
\text { Africa }\end{array}$ & $\begin{array}{l}\text { KR1838 } \\
56\end{array}$ & - & - & - & $\begin{array}{l}\text { Coetzee et } \\
\text { al. } 2015\end{array}$ \\
\hline G. destructans & CMW43671 & $\begin{array}{l}\text { South } \\
\text { Africa }\end{array}$ & $\begin{array}{l}\text { KR1838 } \\
57\end{array}$ & - & - & - & $\begin{array}{l}\text { Coetzee et } \\
\text { al. } 2015\end{array}$ \\
\hline $\begin{array}{l}\text { G. ecuadoriense } \\
\text { A. Salazar, C.W. } \\
\text { Barnes \& Ordoñez }\end{array}$ & $\begin{array}{l}\text { ASL799 } \\
\text { (holotype) }\end{array}$ & Ecuador & $\begin{array}{l}\text { KU1285 } \\
24\end{array}$ & - & - & - & $\begin{array}{l}\text { Crous et al. } \\
2016\end{array}$ \\
\hline G. ecuadoriense & PMC126 & Ecuador & $\begin{array}{l}\text { KU1285 } \\
25\end{array}$ & - & - & - & $\begin{array}{l}\text { Crous et al. } \\
2016\end{array}$ \\
\hline $\begin{array}{l}\text { G. gibbosum } \\
\text { (Cooke) Pat. }\end{array}$ & $\begin{array}{l}\text { SFC20150630- } \\
23\end{array}$ & Korea & $\begin{array}{l}\text { KY3642 } \\
64\end{array}$ & - & $\begin{array}{l}\text { KY3932 } \\
76\end{array}$ & & $\begin{array}{l}\text { Jargalmaa et } \\
\text { al. } 2017\end{array}$ \\
\hline $\begin{array}{l}\text { G. japonicum (Fr.) } \\
\text { Sawada (1931) }\end{array}$ & AS5.69 type 1 & China & $\begin{array}{l}\text { AY5938 } \\
64\end{array}$ & - & - & - & GenBank \\
\hline G. japonicum & AS5.69 type 2 & China & $\begin{array}{l}\text { AY5938 } \\
65\end{array}$ & - & - & - & GenBank \\
\hline G. japonicum & Gja-1 & China & $\begin{array}{l}\text { GU2134 } \\
75\end{array}$ & - & - & - & GenBank \\
\hline G. japonicum & G22 & China & $\begin{array}{l}\text { KX0555 } \\
29\end{array}$ & - & - & - & GenBank \\
\hline $\begin{array}{l}\text { G. leucocontextum } \\
\text { T.H. Li, W.Q. } \\
\text { Deng, Dong M. } \\
\text { Wang \& H.P. Hu }\end{array}$ & Dai15601 & China & $\begin{array}{l}\text { KU5724 } \\
85\end{array}$ & - & - & - & $\begin{array}{l}\text { Xing et al. } \\
2016\end{array}$ \\
\hline $\begin{array}{l}\text { G. } \\
\text { leucocontextum }\end{array}$ & $\begin{array}{l}\text { GDGM40200 } \\
\text { (holotype) }\end{array}$ & China & $\begin{array}{l}\text { KF0115 } \\
48\end{array}$ & - & - & - & Li et al. 2015 \\
\hline $\begin{array}{l}\text { G. lobatum } \\
\text { (Cooke) G.F. Atk. }\end{array}$ & JV1212/10J & USA & $\begin{array}{l}\text { KF6056 } \\
76\end{array}$ & - & - & - & GenBank \\
\hline G. lobatum & JV0409/13J & USA & $\begin{array}{l}\text { KF6056 } \\
75\end{array}$ & - & - & - & GenBank \\
\hline $\begin{array}{l}\text { G. lucidum } \\
\text { (Curtis) P. Karst. }\end{array}$ & K175217 & UK & $\begin{array}{l}\text { KJ14391 } \\
1\end{array}$ & - & $\begin{array}{l}\text { KJ14397 } \\
1\end{array}$ & - & $\begin{array}{l}\text { Zhou et al. } \\
2015\end{array}$ \\
\hline G. lucidum & $\begin{array}{l}\text { MT 26/10 } \\
\text { (BRNM) }\end{array}$ & $\begin{array}{l}\text { Czech } \\
\text { Republic }\end{array}$ & $\begin{array}{l}\text { KJ14391 } \\
2\end{array}$ & - & - & - & $\begin{array}{l}\text { Zhou et al. } \\
2015\end{array}$ \\
\hline $\begin{array}{l}\text { G. mebrekobenum } \\
\text { E.C. Otto, } \\
\text { Blanchette, Held, } \\
\text { C.W. Barnes \& } \\
\text { Obodai }\end{array}$ & $\begin{array}{l}\text { UMN7-3 } \\
\text { GHA(holotype) }\end{array}$ & Ghana & $\begin{array}{l}\text { KX0008 } \\
96\end{array}$ & - & - & - & $\begin{array}{l}\text { Crous et al. } \\
2016\end{array}$ \\
\hline G. mebrekobenum & $\begin{array}{l}\text { UMN7-4GHA } \\
\text { (holotype) }\end{array}$ & Ghana & $\begin{array}{l}\text { KX0008 } \\
98\end{array}$ & - & - & - & $\begin{array}{l}\text { Crous et al. } \\
2016\end{array}$ \\
\hline $\begin{array}{l}\text { G. mizoramense } \\
\text { Zothanz., }\end{array}$ & $\begin{array}{l}\text { UMN-MZ4 } \\
\text { (holotype) }\end{array}$ & India & $\begin{array}{l}\text { KY6437 } \\
50\end{array}$ & - & - & - & $\begin{array}{l}\text { Crous et al. } \\
2017\end{array}$ \\
\hline
\end{tabular}


Table 1 Continued.

\begin{tabular}{|c|c|c|c|c|c|c|c|}
\hline Species & $\begin{array}{l}\text { Voucher } \\
\text { /strain }\end{array}$ & Origin & ITS & LSU & RPB2 & SSU & Reference \\
\hline \multicolumn{8}{|l|}{ Blanchette, Held } \\
\hline G. mizoramense & UMN-MZ5 & India & $\begin{array}{l}\text { KY6437 } \\
51\end{array}$ & $\begin{array}{l}\text { KY643 } \\
751\end{array}$ & - & $\begin{array}{l}\text { KY643 } \\
751\end{array}$ & $\begin{array}{l}\text { Crous et al. } \\
2017\end{array}$ \\
\hline $\begin{array}{l}\text { G. multipileum } \\
\text { Ding Hou }\end{array}$ & $\begin{array}{l}\text { CWN } 04670 \\
\text { (TNM) }\end{array}$ & $\begin{array}{l}\text { Taiwan, } \\
\text { China }\end{array}$ & $\begin{array}{l}\text { KJ14391 } \\
3\end{array}$ & - & $\begin{array}{l}\text { KJ14397 } \\
2\end{array}$ & $\begin{array}{l}\text { KJ1439 } \\
31\end{array}$ & $\begin{array}{l}\text { Zhou et al. } \\
2015\end{array}$ \\
\hline G. multipileum & Dai 9447 (IFP) & $\begin{array}{l}\text { Hainan, } \\
\text { China }\end{array}$ & $\begin{array}{l}\text { KJ14391 } \\
4\end{array}$ & - & $\begin{array}{l}\text { KJ14397 } \\
3\end{array}$ & - & $\begin{array}{l}\text { Zhou et al. } \\
2015\end{array}$ \\
\hline $\begin{array}{l}\text { G. multiplicatum } \\
\text { (Mont.) Pat. }\end{array}$ & Dai 13122 & China & $\begin{array}{l}\text { KU5724 } \\
88\end{array}$ & - & - & - & $\begin{array}{l}\text { Xing et al. } \\
2016\end{array}$ \\
\hline G. multiplicatum & Dai 13710 & China & $\begin{array}{l}\text { KU5724 } \\
89\end{array}$ & - & - & - & $\begin{array}{l}\text { Xing et al. } \\
2016\end{array}$ \\
\hline G. multiplicatum & $\begin{array}{l}\text { GACP1408132 } \\
8\end{array}$ & $\begin{array}{l}\text { Hainan, } \\
\text { China }\end{array}$ & $\begin{array}{l}\text { MH1068 } \\
79\end{array}$ & - & - & - & $\begin{array}{l}\text { Hapuarachch } \\
\text { i et al. } 2018 \mathrm{~b}\end{array}$ \\
\hline $\begin{array}{l}\text { G. orbiforme (Fr.) } \\
\text { Ryvarden }\end{array}$ & $\begin{array}{l}\text { GACP1408095 } \\
3\end{array}$ & $\begin{array}{l}\text { Hainan, } \\
\text { China }\end{array}$ & $\begin{array}{l}\text { MK3131 } \\
08\end{array}$ & - & - & - & This study \\
\hline G. orbiforme & $\begin{array}{l}\text { GACP1408118 } \\
5\end{array}$ & $\begin{array}{l}\text { Hainan, } \\
\text { China }\end{array}$ & $\begin{array}{l}\text { MK3131 } \\
09\end{array}$ & - & - & - & This study \\
\hline $\begin{array}{l}\text { G. podocarpense } \\
\text { J.A. Flores, C.W. } \\
\text { Barnes \& Ordoñez }\end{array}$ & $\begin{array}{l}\text { QCAM6422 } \\
\text { (holotype) }\end{array}$ & Ecuador & $\begin{array}{l}\text { MF7966 } \\
61\end{array}$ & $\begin{array}{l}\text { MF796 } \\
660\end{array}$ & - & - & $\begin{array}{l}\text { Crous et al. } \\
2017\end{array}$ \\
\hline $\begin{array}{l}\text { G. sinense J.D. } \\
\text { Zhao, L.W. Hsu \& } \\
\text { X.Q. Zhang }\end{array}$ & $\begin{array}{l}\text { GACP1709252 } \\
0\end{array}$ & $\begin{array}{l}\text { Sandu, } \\
\text { China }\end{array}$ & $\begin{array}{l}\text { MK3131 } \\
10\end{array}$ & - & - & - & This study \\
\hline G. sinense & $\begin{array}{l}\text { GACP1709252 } \\
2\end{array}$ & $\begin{array}{l}\text { Sandu, } \\
\text { China }\end{array}$ & $\begin{array}{l}\text { MK3131 } \\
11\end{array}$ & - & - & - & This study \\
\hline G. sinense & $\begin{array}{l}\text { GACP1709253 } \\
0\end{array}$ & $\begin{array}{l}\text { Sandu, } \\
\text { China }\end{array}$ & $\begin{array}{l}\text { MK3131 } \\
12\end{array}$ & - & - & $\begin{array}{l}\text { MK341 } \\
557\end{array}$ & This study \\
\hline G. sinense & $\begin{array}{l}\text { GACP1709253 } \\
3\end{array}$ & $\begin{array}{l}\text { Sandu, } \\
\text { China }\end{array}$ & $\begin{array}{l}\text { MK3131 } \\
13\end{array}$ & $\begin{array}{l}\text { MK33 } \\
6399\end{array}$ & - & $\begin{array}{l}\text { MK341 } \\
558\end{array}$ & This study \\
\hline G. sinense & $\begin{array}{l}\text { GACP1709254 } \\
3\end{array}$ & $\begin{array}{l}\text { Sandu, } \\
\text { China }\end{array}$ & $\begin{array}{l}\text { MK3131 } \\
14\end{array}$ & $\begin{array}{l}\text { MK33 } \\
6400\end{array}$ & $\begin{array}{l}\text { MK3714 } \\
31\end{array}$ & $\begin{array}{l}\text { MK341 } \\
559\end{array}$ & This study \\
\hline G. sinense & $\begin{array}{l}\text { GACP1709254 } \\
7\end{array}$ & $\begin{array}{l}\text { Sandu, } \\
\text { China }\end{array}$ & $\begin{array}{l}\text { MK3131 } \\
15\end{array}$ & - & - & - & This study \\
\hline G. sinense & $\begin{array}{l}\text { GACP1709254 } \\
8\end{array}$ & $\begin{array}{l}\text { Sandu, } \\
\text { China }\end{array}$ & $\begin{array}{l}\text { MK3131 } \\
16\end{array}$ & - & - & - & This study \\
\hline G. sinense & $\begin{array}{l}\text { GACP1709256 } \\
7\end{array}$ & $\begin{array}{l}\text { Sandu, } \\
\text { China }\end{array}$ & $\begin{array}{l}\text { MK3131 } \\
17\end{array}$ & $\begin{array}{l}\text { MK33 } \\
6401\end{array}$ & - & $\begin{array}{l}\text { MK341 } \\
560\end{array}$ & This study \\
\hline G. sinense & $\begin{array}{l}\text { GACP1709258 } \\
1\end{array}$ & $\begin{array}{l}\text { Sandu, } \\
\text { China }\end{array}$ & $\begin{array}{l}\text { MK3131 } \\
18\end{array}$ & - & - & - & This study \\
\hline G. sinense & $\begin{array}{l}\text { GACP1709258 } \\
8\end{array}$ & $\begin{array}{l}\text { Sandu, } \\
\text { China }\end{array}$ & $\begin{array}{l}\text { MK3131 } \\
19\end{array}$ & $\begin{array}{l}\text { MK33 } \\
6402\end{array}$ & $\begin{array}{l}\text { MK3714 } \\
32\end{array}$ & $\begin{array}{l}\text { MK341 } \\
561\end{array}$ & This study \\
\hline G. sinense & $\begin{array}{l}\text { GACP1709259 } \\
2\end{array}$ & $\begin{array}{l}\text { Sandu, } \\
\text { China }\end{array}$ & $\begin{array}{l}\text { MK3131 } \\
20\end{array}$ & & - & - & This study \\
\hline G. sinense & $\begin{array}{l}\text { GACP1709251 } \\
09\end{array}$ & $\begin{array}{l}\text { Sandu, } \\
\text { China }\end{array}$ & $\begin{array}{l}\text { MK3131 } \\
21\end{array}$ & - & - & - & This study \\
\hline G. sinense & $\begin{array}{l}\text { GACP1709251 } \\
28\end{array}$ & $\begin{array}{l}\text { Sandu, } \\
\text { China }\end{array}$ & $\begin{array}{l}\text { MK3131 } \\
22\end{array}$ & - & - & - & This study \\
\hline G. sinense & $\begin{array}{l}\text { GACP1709251 } \\
30\end{array}$ & $\begin{array}{l}\text { Sandu, } \\
\text { China }\end{array}$ & $\begin{array}{l}\text { MK3131 } \\
23\end{array}$ & & & & \\
\hline G. sinense & $\begin{array}{l}\text { GACP1710124 } \\
2\end{array}$ & Kaili, China & $\begin{array}{l}\text { MK3131 } \\
24\end{array}$ & - & - & - & This study \\
\hline G. sinense & $\begin{array}{l}\text { GACP1710125 } \\
4\end{array}$ & Kaili, China & $\begin{array}{l}\text { MK3131 } \\
25\end{array}$ & $\begin{array}{l}\text { MK33 } \\
6403\end{array}$ & - & $\begin{array}{l}\text { MK341 } \\
562\end{array}$ & This study \\
\hline
\end{tabular}


Table 1 Continued.

\begin{tabular}{|c|c|c|c|c|c|c|c|}
\hline Species & $\begin{array}{l}\text { Voucher } \\
\text { /strain }\end{array}$ & Origin & ITS & LSU & RPB2 & SSU & Reference \\
\hline G. sinense & $\begin{array}{l}\text { GACP1710126 } \\
0\end{array}$ & Kaili, China & $\begin{array}{l}\text { MK3131 } \\
26\end{array}$ & $\begin{array}{l}\text { MK33 } \\
6404\end{array}$ & - & $\begin{array}{l}\text { MK341 } \\
563\end{array}$ & This study \\
\hline G. sinense & $\begin{array}{l}\text { GACP1710127 } \\
0\end{array}$ & Kaili, China & $\begin{array}{l}\text { MK3131 } \\
27\end{array}$ & $\begin{array}{l}\text { MK33 } \\
6405\end{array}$ & - & $\begin{array}{l}\text { MK341 } \\
564\end{array}$ & This study \\
\hline G. sinense & $\begin{array}{l}\text { GACP1710122 } \\
39\end{array}$ & Kaili, China & $\begin{array}{l}\text { MK3131 } \\
28\end{array}$ & - & - & - & This study \\
\hline G. sinense & $\begin{array}{l}\text { GACP1408123 } \\
6\end{array}$ & $\begin{array}{l}\text { Hainan, } \\
\text { China }\end{array}$ & $\begin{array}{l}\text { MH1068 } \\
82\end{array}$ & - & - & - & $\begin{array}{l}\text { Hapuarachch } \\
\text { i et al. } 2018 \mathrm{~b}\end{array}$ \\
\hline G. sinense & Wei5327 & $\begin{array}{l}\text { Hainan, } \\
\text { China }\end{array}$ & $\begin{array}{l}\text { KF4949 } \\
98\end{array}$ & $\begin{array}{l}\text { KF495 } \\
008\end{array}$ & $\begin{array}{l}\text { MG3675 } \\
29\end{array}$ & - & GenBank \\
\hline G. sinense & Cui13835 & $\begin{array}{l}\text { Hainan, } \\
\text { China }\end{array}$ & $\begin{array}{l}\text { MG2791 } \\
93\end{array}$ & - & $\begin{array}{l}\text { MG3675 } \\
30\end{array}$ & & $\begin{array}{l}\text { Xing et al. } \\
2018\end{array}$ \\
\hline G. sinense & GDGM25829 & China & $\begin{array}{l}\text { KC4157 } \\
60\end{array}$ & & & & GenBank \\
\hline G. sinense & GS96 & China & $\begin{array}{l}\text { DQ4249 } \\
90\end{array}$ & - & - & - & GenBank \\
\hline G. sinense & GS92 & China & $\begin{array}{l}\text { DQ4249 } \\
82\end{array}$ & - & - & - & GenBank \\
\hline G. sinense & GS175 & China & $\begin{array}{l}\text { DQ4250 } \\
14\end{array}$ & - & - & - & GenBank \\
\hline G. sinense & GS111 & China & $\begin{array}{l}\text { DQ4249 } \\
95\end{array}$ & - & - & - & GenBank \\
\hline G. sinense & XZ-G-C1 & China & $\begin{array}{l}\text { HQ2356 } \\
33\end{array}$ & & - & & GenBank \\
\hline G. sinense & XZ-G-C2 & China & $\begin{array}{l}\text { HQ2356 } \\
34\end{array}$ & & - & & GenBank \\
\hline G. sinense & $\mathrm{ZZ}$ & China & $\begin{array}{l}\text { KM2499 } \\
33\end{array}$ & - & - & - & GenBank \\
\hline $\begin{array}{l}\text { G. tropicum } \\
\text { (Jungh.) Bres. }\end{array}$ & Dai9724 & China & $\begin{array}{l}\text { JQ78187 } \\
9\end{array}$ & - & - & - & $\begin{array}{l}\text { Cao et al. } \\
2012\end{array}$ \\
\hline G. tropicum & $\begin{array}{l}\text { GACP1408151 } \\
8\end{array}$ & China & $\begin{array}{l}\text { MH1068 } \\
84\end{array}$ & - & - & - & $\begin{array}{l}\text { Hapuarachch } \\
\text { i et al. } 2018 \mathrm{~b}\end{array}$ \\
\hline $\begin{array}{l}\text { G. wiiroense E.C. } \\
\text { Otto, Blanchette, } \\
\text { C.W. Barnes \& } \\
\text { Held }\end{array}$ & $\begin{array}{l}\text { UMN-20 } \\
\text { (holotype) } \\
\text { GHA }\end{array}$ & Ghana & $\begin{array}{l}\text { KT9523 } \\
61\end{array}$ & - & - & - & $\begin{array}{l}\text { Crous et al. } \\
2015\end{array}$ \\
\hline G. wiiroense & $\begin{array}{l}\text { UMN-21-GHA } \\
\text { (para type) }\end{array}$ & Ghana & $\begin{array}{l}\text { KT9523 } \\
63\end{array}$ & - & - & - & $\begin{array}{l}\text { Crous et al. } \\
2015\end{array}$ \\
\hline $\begin{array}{l}\text { Coriolopsis trogii } \\
\text { (Berk.) Domański }\end{array}$ & RLG4286sp & USA & $\begin{array}{l}\text { JN16499 } \\
3\end{array}$ & - & $\begin{array}{l}\text { JN16486 } \\
7\end{array}$ & - & $\begin{array}{l}\text { Jargalmaa et } \\
\text { al. } 2017\end{array}$ \\
\hline
\end{tabular}

\section{Results}

\section{Phylogeny}

The tree topologies obtained from ML, MP and BY were identical, therefore, only the ML tree is shown (Fig. 1). Our twenty collections of Ganoderma sinense from China clustered with all $G$. sinense and $G$. japonicum sequences, in a well-supported clade $(\mathrm{ML}=100 \%, \mathrm{MP}=99 \%, \mathrm{BPP}=$ 1.00).

\section{Taxonomy}

Ganoderma P. Karst., Rev. Mycol. (Toulouse) 3: 17 (1881)

= Dendrophagus Murrill, Bull. Torrey bot. Club 32(9): 473 (1905) 
= Elfvingia P. Karst., Bidr. Känn. Finl. Nat. Folk 48: 333 (1889)

= Friesia Lázaro Ibiza, Revista Real Acad. Ci. Madrid 14: 587 (1916)

= Ganoderma subgen. Trachyderma Imazeki, Bull. Tokyo Sci. Mus.1: 49 (1939)

= Tomophagus Murrill, Torreya 5: 197 (1905)

= Trachyderma (Imazeki) Imazeki, Bull. Gov. Forest Exp. Stn Tokyo 57: 97 (1952)

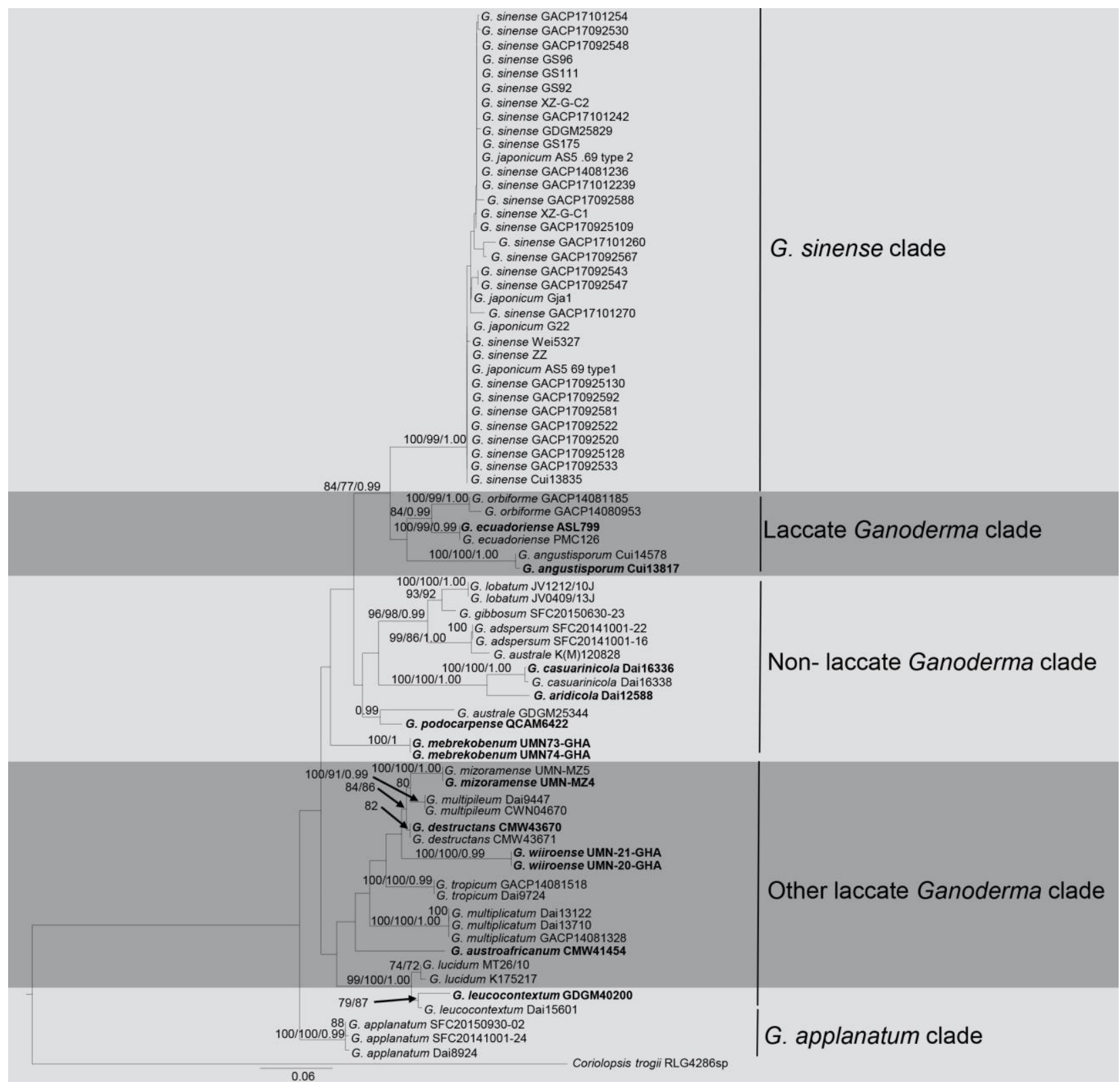

Fig. 1 - Phylogram for Ganoderma generated from maximum likelihood analysis of ITS, LSU, SSU and RPB2 sequence data. Bootstrap support values for maximum likelihood and maximum parsimony, greater than $70 \%$ and posterior probabilities from Bayesian inference $\geq 0.95$ are given above branches. The tree is rooted with Coriolopsis trogii. Type species are indicated in bold.

Description (from Ryvarden 2004).

Basidiomes annual or perennial, stipitate to sessile; pileus surface with a thick, dull cuticle or shiny and laccate with a thin cuticle or cuticle of clavate end cells; context cream coloured to dark purplish brown, soft and spongy to firm-fibrous; pore surface cream coloured, bruising brown, the pores regular, 4-7 per $\mathrm{mm}$; tube layers single or stratified, pale to purplish brown; stipe when present central or lateral; hyphal system dimitic; generative hyphae with clamps; skeletal hyphae hyaline to brown, non-septate, often with long, tapering branches; basidia broadly ellipsoid, 
tapering abruptly at the base; cystidia absent; basidiospores broadly to narrowly ellipsoid with a truncate apex and apical germ pore, wall two-layered, endosporium brown and separated from the hyaline exosporium by inter-wall pillars, negative in Melzer's reagent, 7-30 $\mu \mathrm{m}$ long.

Type species - Ganoderma lucidum (Leyss: Fr.) Karst.

Ganoderma sinense J.D. Zhao, L.W. Hsu \& X.Q. Zhang, Acta Microbiol. Sin. 19(3): 272 (1979)

Figs 2-19

= Ganoderma formosanum T.T. Chang \& T. Chen, Trans. Or. Mycol. Soc. 82: 731 (1984). Misapplications:

= Ganoderma japonicum (Fr.) Lloyd in Teng, Fungi of China: 447 (1963); Tai, Syll. Fung. Sin.: 469 (1979); Teng, Fungi of China: 326 (1996), non Polyporus japonicus Fr., Epicrisis: 442 (1838) (Ganoderma japonicum (Fr.) Lloyd, Mycol. Writ. 3: Syn. Stip. Polyp.: 102(1912).

= Ganoderma lucidum (Leyss.) P. Karst. var. japonicum (Fr.) Bres. in Teng, Sinensia 5: 199 (1934). non Polyporus japonicus Fr., Epicrisis: 442 (1838).

Description (Wang et al. 2005): Basidiomes annual, stipitate, corky-woody. Pileus $2.5-0.5 \times$ $5.2-9 \mathrm{~cm} .9 .2-1.2 \mathrm{~cm}$ thick in nature, $2.5-6.5 \times 3.5-12 \mathrm{~cm} .0 .5-1.5 \mathrm{~cm}$ thick when cultivated, dimidiate; upper surface usually purplish black to black, laccate, concentrically sulcate or not, radially rugose; margin often subtruncated. Pore surface pale brown to dark brown: tubes up to 1.4

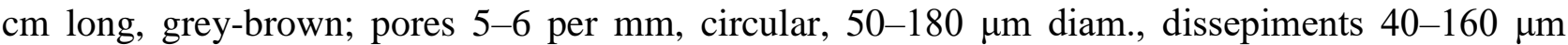
thick. Stipe 6-19 cm long, $0.5-1.0 \mathrm{~cm}$ thick, lateral, dorsolateral or eccentric, cylindrical or flattened: concolorous with the pileus, laccate. Context 1-5 mm thick, uniformly brown or red brown near the tube layer or with whitish streaks and patches near cutis; hyphal system trimitic; generative hyphae 3-5 $\mu \mathrm{m}$ diam., hyaline, thin-walled, with clamp connections; skeletal hyphae 4.5-7 $\mu \mathrm{m}$ diam., golden brown in 5\% $\mathrm{KOH}$ solution, dextrinoid in Molder's reagent; ligative hyphae 1-2.5 $\mu \mathrm{m}$ diam., thick-walled, much branched. Basidiospores 10.5-13.5 $\times 7-9 \mu \mathrm{m}$ including endosporium and 8-9 $\times$ 5.5-7. $\mu \mathrm{m}$, excluding myxosporium: ovoid; brown with a dark brown eusporium bearing few and thick echinulae, overlaid by a hyaline myxosporium, truncate or not at the apex. Basidia not seen. Cutis hymeniodermic elements $20-60 \times 4-8 \mu \mathrm{m}$, clavate, amyloid in Melzer's reagent.

\section{Annotated list of Ganoderma sinense specimens with different morphological characteristics}

We have collected 20 morphologically different $G$. sinense specimens from China and collection sites details are summarized in Table 3. Detailed morphological descriptions of each specimen are as follows.

\section{Specimen no. 1}

Basidiome annual, stipitate, strongly laccate, corky. Pileus $7-8.5 \times 6-8 \mathrm{~cm}$, up to $1 \mathrm{~cm}$ thick at the base, orbicular, upper surface brownish black (6C8) to brown (6E4) pale brown (5A5) at the margin, concentrically sulcate zones, irregularly ruptured crust overlying the context; margin $1 \mathrm{~mm}$ thick, blunt, pale brown (5A5); lower surface pale brown (5A5). Hymenophore up to $12 \mathrm{~mm}$ long, indistinctly stratose; pores brownish grey (6D2), pores circular, sub circular or isodiametric, 4-5 per mm. Context up to $1.5 \mathrm{~cm}$ thick, duplex, dry, upper layer dark brown (7F8), corky; lower layer pale brown (5A5), fibrous, composed of coarse loose fibrils, woody. Stipe central, sub cylindrical, concolorous with the pileus, $8 \times 13 \mathrm{~cm}, 1.5 \mathrm{~cm}$ at the base. Basidiospores $(\mathrm{n}=20)(8.7-) 10.1-$ $11.5-13(14.1-) \times(-5.3) 7-7.9-8.9(-9.2) \mu \mathrm{m}\left(Q_{m}=1.5, Q=1.2-2.1\right.$, with myxosporium $)$. (6.9-)8.19.2-10.2(-10.7) $\times(3.4-) 5.3-6.4-7.5(-7.8) \mu \mathrm{m}\left(Q_{m}=1.4, Q=1.2-2.1\right.$, without myxosporium), ellipsoid, brown (6E4), with a pale brown (5A5) eusporium bearing fine, short and distinct echinulae, overlaid by a hyaline myxosporium. Pileipellis a hymeniderm, brown (6E4), clavate like cells. Context trimitic; generative hyphae $(\mathrm{n}=20)(0.8-1.1-1.6) \mu \mathrm{m}$ in width, colorless, thinwalled; skeletal hyphae $(\mathrm{n}=20)(2.8-3.4-3.8) \mu \mathrm{m}$ in width, thick walled, nearly solid, sometimes branched, brown (6E4); binding hyphae $(\mathrm{n}=20)(3.2-4.4-5.4) \mu \mathrm{m}$ in width, thick walled, branched, nearly solid, brown (6E4) (Fig. 2) 


\section{Specimen no. 2}

Basidiome annual, stipitate, strongly laccate, corky. Pileus $12-15.5 \times 10-12.5 \mathrm{~cm}$, up to 1.5 $\mathrm{cm}$ thick at the base, subreniform; upper surface reddish brown (8E7) to brownish orange (6C8), distinctly concentrically sulcate zones, irregularly ruptured crust overlying the context, swollen at the point of attachment; margin $2 \mathrm{~mm}$ thick, blunt, concolorous with the pileus; lower surface pale brown (5A5). Hymenophore up to $12 \mathrm{~mm}$ long, indistinctly stratose; pores brownish grey (6D2), circular or sub circular, 3-5 per mm. Context up to $1 \mathrm{~cm}$ thick, duplex, dry; upper layer dark brown (7F8), corky; lower layer pale brown (5A5), fibrous, composed of coarse loose fibrils, woody. Stipe eccentric, sub cylindrical, concolorous with the pileus, $8 \times 15 \mathrm{~cm}, 1 \mathrm{~cm}$ at the base. Basidiospores $(\mathrm{n}=25)(10.6-) 11.5-12.5-13.5(14.3-) \times(-7.1) 7.7-8.3-8.9(-11.7) \mu \mathrm{m} Q_{m}=1.5, Q=1.1-1.8$, with myxosporium). (6.8-)9.2-10.2-11.1(-11.7) × (5.4-)6.1-6.8-7.7(-8.4) $\mu \mathrm{m}\left(Q_{m}=1.5, Q=1.1-1.8\right.$, without myxosporium), ellipsoid, brown (6E4), with a pale brown (5A5) eusporium bearing fine, short and distinct echinulae, overlaid by a hyaline myxosporium. Pileipellis a hymeniderm, brown (6E4), clavate like cells. Context trimitic, generative hyphae $(n=25)(0.5-1.6-3.3) \mu \mathrm{m}$ in width, colorless, thin-walled; skeletal hyphae $(\mathrm{n}=20)(3.3-4.5-5.2) \mu \mathrm{m}$ in width, thick walled, nearly solid, sometimes branched, brown (6E4); binding hyphae $(\mathrm{n}=20)(3.4-4.6-5.5) \mu \mathrm{m}$, thick walled, branched, nearly solid, brown (6E4) (Fig. 3).

\section{Specimen no. 3}

Basidiome annual, stipitate, laccate, corky. Pileus $5.5-8 \times 3-4.5 \mathrm{~cm}$, up to $0.5 \mathrm{~cm}$ thick at the base, sub orbicular; upper surface brown (6E4) to brownish black (6C8), yellowish brown (5D8) at the margin, slightly radially rugose, irregularly ruptured crust overlying the context; margin $1 \mathrm{~mm}$ thick, soft, yellow brown (5D8); lower surface light brown (6D6). Hymenophore up to $12 \mathrm{~mm}$ long, indistinctly stratose; pores brownish grey (6D2), circular, sub circular or isodiametric, 3-5 per mm. Context up to $3 \mathrm{~mm}$ thick, duplex, dry; upper layer dark brown (7F8), corky; lower layer pale brown (5A5), fibrous, composed of coarse loose fibrils; woody. Stipe eccentric, sub cylindrical, concolorous with the pileus, $5.5 \times 12 \mathrm{~cm}, 1 \mathrm{~cm}$ thick at the base. Basidiospores $(\mathrm{n}=20)(7.5-) 8.7-$ 10.2-11.6(12.2-) $\times(-5.2) 6.2-7.2-8.3(-8.9) \mu \mathrm{m}\left(Q_{m}=1.4, Q=1.2-1.7\right.$, with myxosporium). (5.76.5-7.7-9(-9.5) × (3.7-)4.5-5.4-6.3(-6.5) $\mu \mathrm{m}\left(Q_{m}=1.4, Q=1.2-1.7\right.$, without myxosporium), ellipsoid, brown (6E4), with a pale brown (5A5), eusporium bearing fine, short and distinct echinulae, overlaid by a hyaline myxosporium. Pileipellis a hymeniderm, brown (6E4), clavate like cells. Context trimitic; generative hyphae $(\mathrm{n}=20)(1.3-2.1-3.5) \mu \mathrm{m}$ in width, colourless, thin walled without clamp connections; skeletal hyphae $(\mathrm{n}=20)(1.8-3.9-6.1) \mu \mathrm{m}$ in width, thick walled, nearly solid, sometimes branched, brown (6E4); binding hyphae $(\mathrm{n}=20)(2.7-4.2-6.5) \mu \mathrm{m}$ in width, thick walled, branched, nearly solid, brown (6E4) (Fig. 4).

\section{Specimen no. 4}

Basidiome annual, stipitate, strongly laccate, branched, corky. Pileus 6-10 $\times 5-8 \mathrm{~cm}$, up to 1 $\mathrm{cm}$ thick at the base, orbicular; upper surface brown (6E4) to brownish black (6C8), yellowish brown (5D8) at the margin, irregularly ruptured crust overlying the context; margin blunt, yellow brown (5D8); lower surface light brown (6D6). Hymenophore up to $20 \mathrm{~mm}$ long, indistinctly stratose; pores brownish grey (6D2), circular, 2-4 per mm. Context up to $1 \mathrm{~cm}$ thick, duplex, dry, upper layer dark brown (7F8), corky; lower layer pale brown (5A5), fibrous, composed of coarse loose fibrils; woody. Stipe central, sub cylindrical, concolorous with the pileus, $5 \times 8 \mathrm{~cm}, 1.5 \mathrm{~cm}$ at the base. Basidiospores $(\mathrm{n}=20)(8-) 9.7-11.6-11.2(13.4-) \times(-6.3) 7.3-8.1-8.8(-9.6) \mu \mathrm{m}\left(Q_{m}=\right.$ $1.4, Q=1.1-1.6$, with myxosporium). (6.6-)8.2-9.6-10.9(-11.4) $\times(5.8-) 7.3-6.7-7.5(-8.5) \mu \mathrm{m}$ $\left(Q_{m}=1.4, Q=1.2-1.7\right.$, without myxosporium), ellipsoid, brown (6E4), with a pale brown (5A5) eusporium bearing fine, short and distinct echinulae, overlaid by a hyaline myxosporium. Pileipellis a hymeniderm, brown (6E4), clavate like cells, dextrinoid. Context trimitic; generative hyphae $(\mathrm{n}=20)(0.3-1.3-2.2) \mu \mathrm{m}$ in width, colorless, thin-walled; skeletal hyphae $(\mathrm{n}=20)(2.6-$ 3.6-5.1) $\mu \mathrm{m}$ in width, thick walled, nearly solid, sometimes branched, brown (6E4); binding 
hyphae $(\mathrm{n}=20)(0.9-2.6-4.5) \mu \mathrm{m}$ in width, thick walled, branched, nearly solid, pale brown (5A5) (Fig. 5).
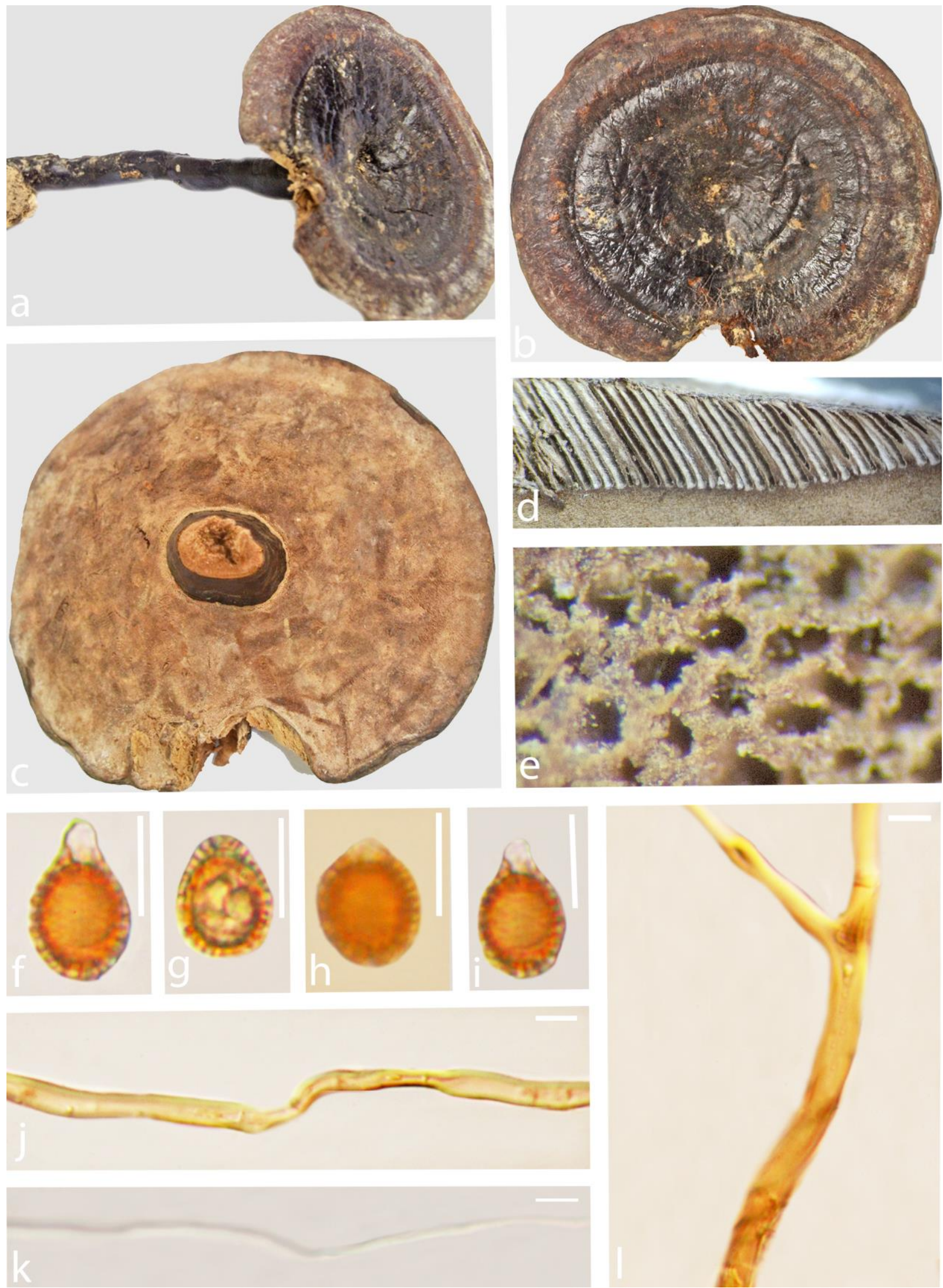

Fig. 2 - Ganoderma sinense specimen no. 1 (GACP17092567). a, b Upper surface. c Lower surface. d Cut surface. e Pores in the lower surface $(5 \times)$. f-i Spores (100x). j Skeletal hyphae (100x). k Generative hyphae (100x). 1 Binding hyphae (100x). Scale bars: $f-i=10 \mu \mathrm{m}, \mathrm{j}-1=5 \mu \mathrm{m}$. 

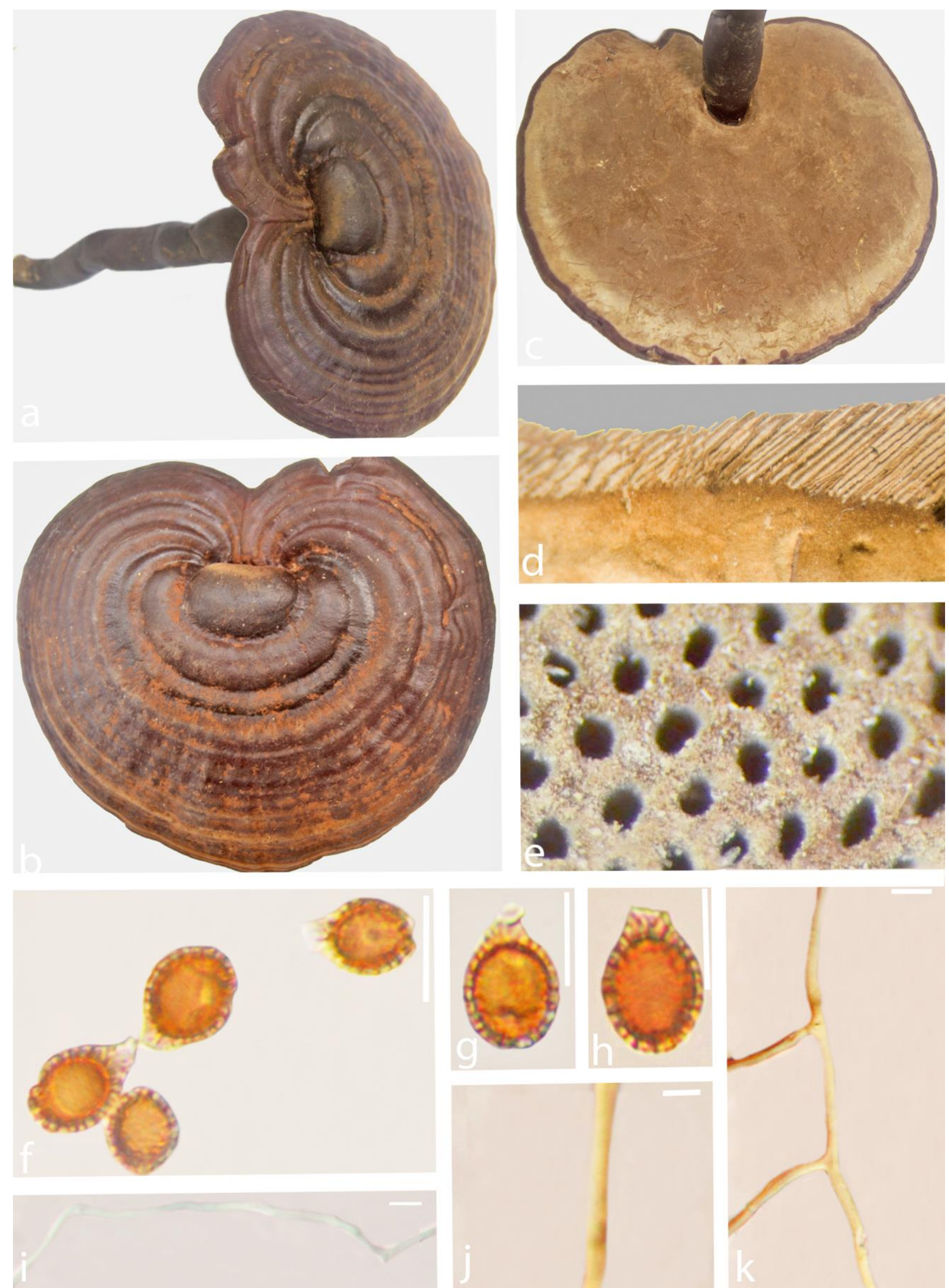

Fig. 3 - Ganoderma sinense specimen no. 2 (GACP17092533). a, b Upper surface. c Lower surface. d Cut surface. e Pores in the lower surface $(5 \times)$. $\mathrm{f}-\mathrm{h}$ Spores $(100 \times)$. i Generative hyphae (40x). j Skeletal hyphae (40x). k Binding hyphae (40x). Scale bars: $\mathrm{f}-\mathrm{h}=10 \mu \mathrm{m}, \mathrm{i}-\mathrm{k}=5 \mu \mathrm{m}$. 

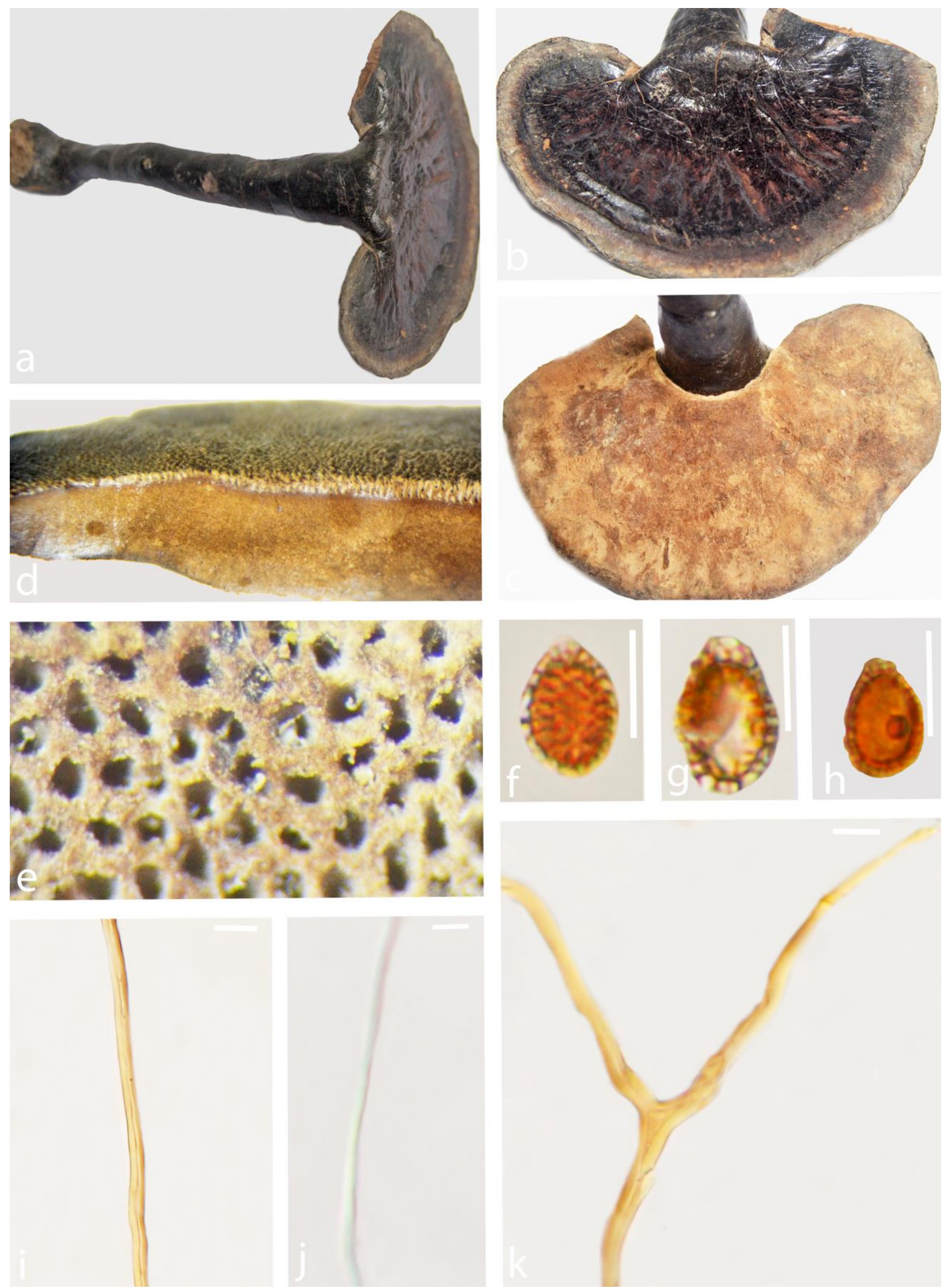

Fig. 4 - Ganoderma sinense specimen no. 3 (GACP17092522). a, b Upper surface. c Lower surface. d Cut surface. e Pores in the lower surface $(5 \times)$. f-h Spores $(100 x)$. i Skeletal hyphae $(100 \times)$. j Generative hyphae (100x). k Binding hyphae (100x). Scale bars: $\mathrm{f}-\mathrm{h}=10 \mu \mathrm{m}, \mathrm{i}-\mathrm{k}=5 \mu \mathrm{m}$. 

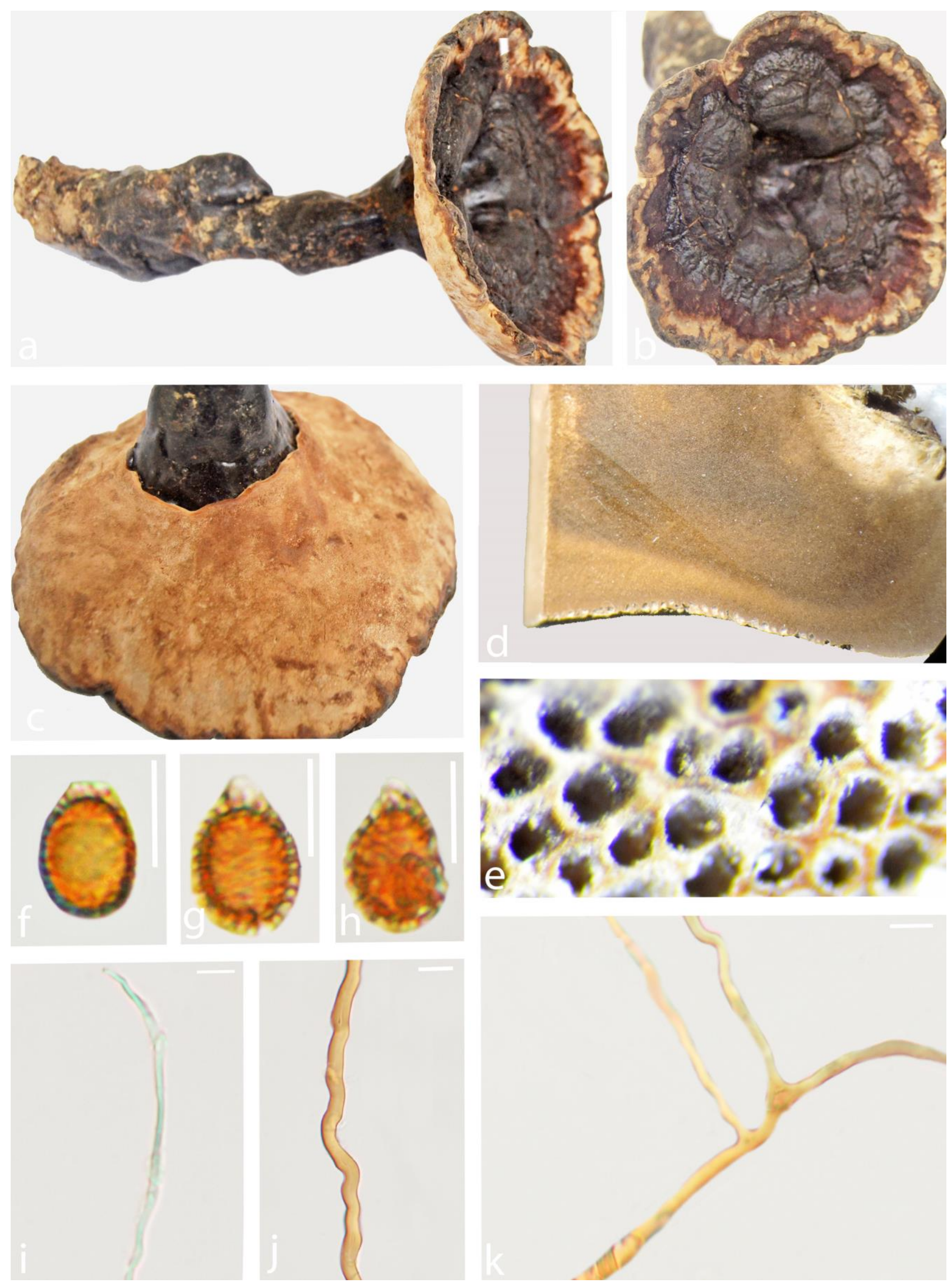

Fig. 5 - Ganoderma sinense specimen no. 4 (GACP17092530). a, b Upper surface. c Lower surface. d Cut surface. e Pores in the lower surface $(5 x)$. $\mathrm{f}-\mathrm{h}$ Spores $(100 \times)$. i Generative hyphae $(100 \times)$. j Skeletal hyphae $(100 \times)$. k Binding hyphae $(100 \times)$. Scale bars: $\mathrm{f}-\mathrm{h}=10 \mu \mathrm{m}, \mathrm{i}-\mathrm{k}=5 \mu \mathrm{m}$. 


\section{Specimen no. 5}

Basidiome annual, stipitate, strongly laccate, corky. Pileus $7-9 \times 7-8 \mathrm{~cm}$, up to $1.5 \mathrm{~cm}$ thick at the base, spathulate, suborbicular to irregular shape; upper surface brownish black (6C8), irregularly ruptured crust overlying the context; margin $3 \mathrm{~mm}$ thick, blunt, concolourous with the pileus; lower surface light brown (6D6). Hymenophore up to $20 \mathrm{~mm}$ long, indistinctly stratose; pores brownish grey (6D2), pores circular, 3-5 per mm. Context up to $1 \mathrm{~cm}$ thick, duplex, dry, upper layer dark brown (7F8); lower layer pale brown (5A5), fibrous, composed of coarse loose fibrils; woody corky. Stipe eccentric, sub cylindrical, concolorous with the pileus, $8 \times 12 \mathrm{~cm}, 2.5$ $\mathrm{cm}$ at the base. Basidiospores $(\mathrm{n}=25)(9.5-) 11.1-12.3-13.5(14.5-) \times(-7.1) 8-8.6-9.3(-9.9) \mu \mathrm{m}$ $\left(Q_{m}=1.4, \mathrm{Q}=1.1-1.7\right.$, with myxosporium). (7.5-)9-9.8-10.7(-11) $\times(4.8-) 6.3-7.1-7.8(-9.1) \mu \mathrm{m}$ ( $Q_{m}=1.4, Q=1.1-1.9$, without myxosporium), ellipsoid, brown (6E4), with a pale brown (5A5), eusporium bearing fine, short and distinct echinulae, overlaid by a hyaline myxosporium. Pileipellis a hymeniderm, brownish orange (6C4), clavate like cells, dextrinoid. Context trimitic; generative hyphae $(\mathrm{n}=20)(0.7-1.1-1.3) \mu \mathrm{m}$ in width, colorless, thin-walled; skeletal hyphae $(\mathrm{n}=$ 20) $(2.1-3.6-2.9) \mu \mathrm{m}$ in width, thick walled, nearly solid, sometimes branched, brown (6E4); binding hyphae $(\mathrm{n}=22)(1.7-2.6-3.8) \mu \mathrm{m}$ in width, thick walled, branched, nearly solid, brown (6E4) (Fig. 6).

\section{Specimen no. 6}

Basidiome annual, stipitate, strongly laccate, branched, corky. Pileus 6-10 $\times 5-8 \mathrm{~cm}$, up to 1 $\mathrm{cm}$ thick at the base, subreniform; upper surface grey $(8 \mathrm{~F} 1)$ to brownish grey (8E2), radially rugose, concentrically sulcate zone, irregularly ruptured crust overlying the context, margin; $3 \mathrm{~mm}$ thick, blunt, concolourous with the pileus, lower surface; reddish brown (8E6). Hymenophore up to $20 \mathrm{~mm}$ long, indistinctly stratose; pores initially brownish grey (8D2), reddish brown (8E7) to dark brown (8F6), fibrous, composed of coarse loose fibrils, corky; lower layer brownish red (8C8), woody. Stipe eccentric, sub cylindrical, dichotomous to trichotomous to irregularly branched, concolorous with the pileus, $5 \times 8 \mathrm{~cm}, 1.5 \mathrm{~cm}$ from the base. Basidiospores $(\mathrm{n}=20)(9.2-) 9.8-$ 11.2-12.5(12.6-) $\times(-6.4) 7.3-8.1-8.7(-8.8) \mu \mathrm{m},\left(Q_{m}=1.4, Q=1.1-1.4\right.$, with myxosporium $) .(\mathrm{n}=$ 20) (7.2-)7.8-9.1-10.3(-10.8) × (5.5-)6.2-6.7-6.8(-7.4) $\mu \mathrm{m},\left(Q_{m}=1.3, Q=1.2-1.5\right.$, without myxosporium), broadly ellipsoid, brown (6D6), with a light brown (6D6) eusporium bearing thick, short and distinct echinulae, overlaid by a hyaline myxosporium. Pileipellis a hymeniderm, brownish orange $(6 \mathrm{C} 8)$, clavate like cells. Context trimitic; generative hyphae $(\mathrm{n}=20)(1.2-1.5-$ 1.8) $\mu \mathrm{m}$ in width, colorless, thin-walled; skeletal hyphae $(\mathrm{n}=25)(2.1-2.8-3.8) \mu \mathrm{m}$ in width, thick walled, nearly solid, sometimes branched, brown (6D6); binding hyphae $(\mathrm{n}=20)(2.1-2.8-4.1) \mu \mathrm{m}$ in width, thick walled, branched, nearly solid, brown (6D6) (Fig. 7).

\section{Specimen no. 7}

Basidiome annual, stipitate, laccate, corky. Pileus 6-8 $\times 3.5-5.5 \mathrm{~cm}$, up to $1 \mathrm{~cm}$ thick at the base, sub reniform, upper surface; brownish grey (6C2) to black, radially rugose with turberculate bumps and ridges and rivulose depressions, distinctly concentrically sulcate zones, irregularly ruptured crust overlying the context, swollen at the point of attachment; margin blunt, concolourous with the pileus, 3mm thick; lower surface light brown (6D6). Hymenophore up to $15 \mathrm{~mm}$ long, indistinctly stratose; pores initially brownish grey (6D2), bruising dark brown (6F6), pores circular or sub circular, 4-6 per mm. Context up to $1 \mathrm{~cm}$ thick, dry, duplex; upper layer brown (6E6) to dark brown (6F6), fibrous/pithy, composed of coarse loose fibrils; lower layer brown (6D8), woody. Stipe dorsally lateral to nearly dorsal, sub cylindrical, concolorous with the pileus, $6 \times 10 \mathrm{~cm}, 1 \mathrm{~cm}$ at the base. Basidiospores $(\mathrm{n}=25)(8.7-) 9.9-11.0-12.1(12.7-) \times(-5.6) 6.8-7.5-8.1(-8.2) \mu \mathrm{m},\left(Q_{m}\right.$ $=1.5, Q=1.2-2.6$, with myxosporium $)$. $(\mathrm{n}=25)(6.1-) 7.2-8.2-9.2(-9.7) \times(4.1-) 4.9-5.6-6.4(-$ 6.8) $\mu \mathrm{m},\left(Q_{m}=1.5, Q=1.1-1.8\right.$, without myxosporium), ellipsoid, brownish orange (6C8) with a brown (6E6), eusporium bearing thick, short and distinct echinulae, overlaid by a hyaline myxosporium. Pileipellis a hymeniderm, brownish orange (6C8), clavate like cells. Context trimitic; generative hyphae $(\mathrm{n}=20)(0.5-1.2-3.1) \mu \mathrm{m}$ in width, hyaline, thin walled without clamp 
connections; skeletal hyphae $(\mathrm{n}=20)(1.3-2-3.1) \mu \mathrm{m}$ in width, thick walled, nearly solid, sometimes branched, brown (6E6) to dark brown (6F6); binding hyphae $(\mathrm{n}=30)(0.9-2.2-4.6) \mu \mathrm{m}$ in width, thick walled, branched, nearly solid, brown (6E6) to dark brown (6F6) (Fig. 8).
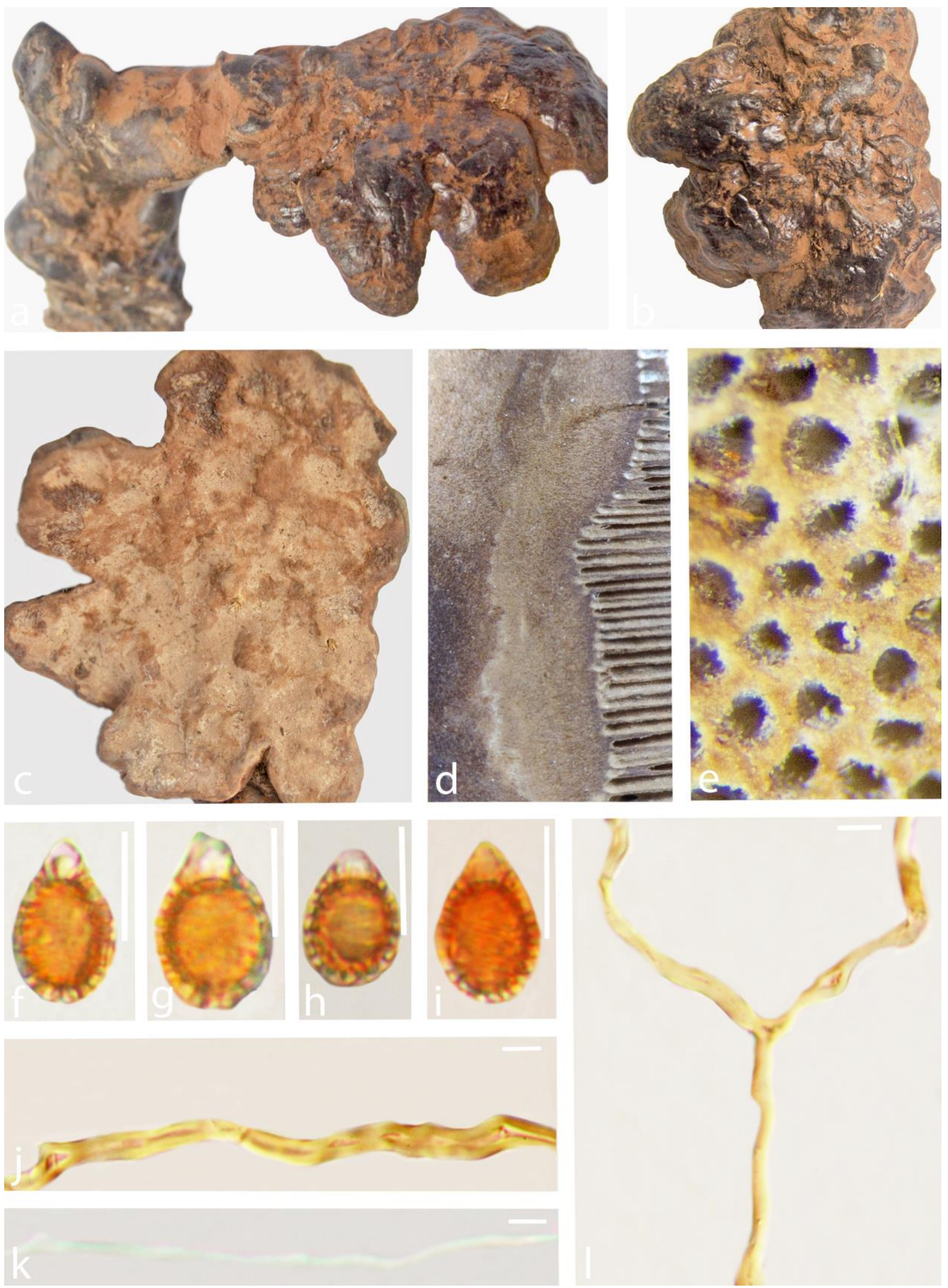

Fig. 6 - Ganoderma sinense specimen no. 5 (GACP170925128). a, b Upper surface. c Lower surface. d Cut surface. e Pores in the lower surface (5x). f-i spores (100x). j Skeletal hyphae $(100 \times)$. k Generative hyphae $(100 \times)$. 1 Binding hyphae $(100 \times)$. Scale bars: $f-i=10 \mu \mathrm{m}, j-1=5 \mu \mathrm{m}$. 

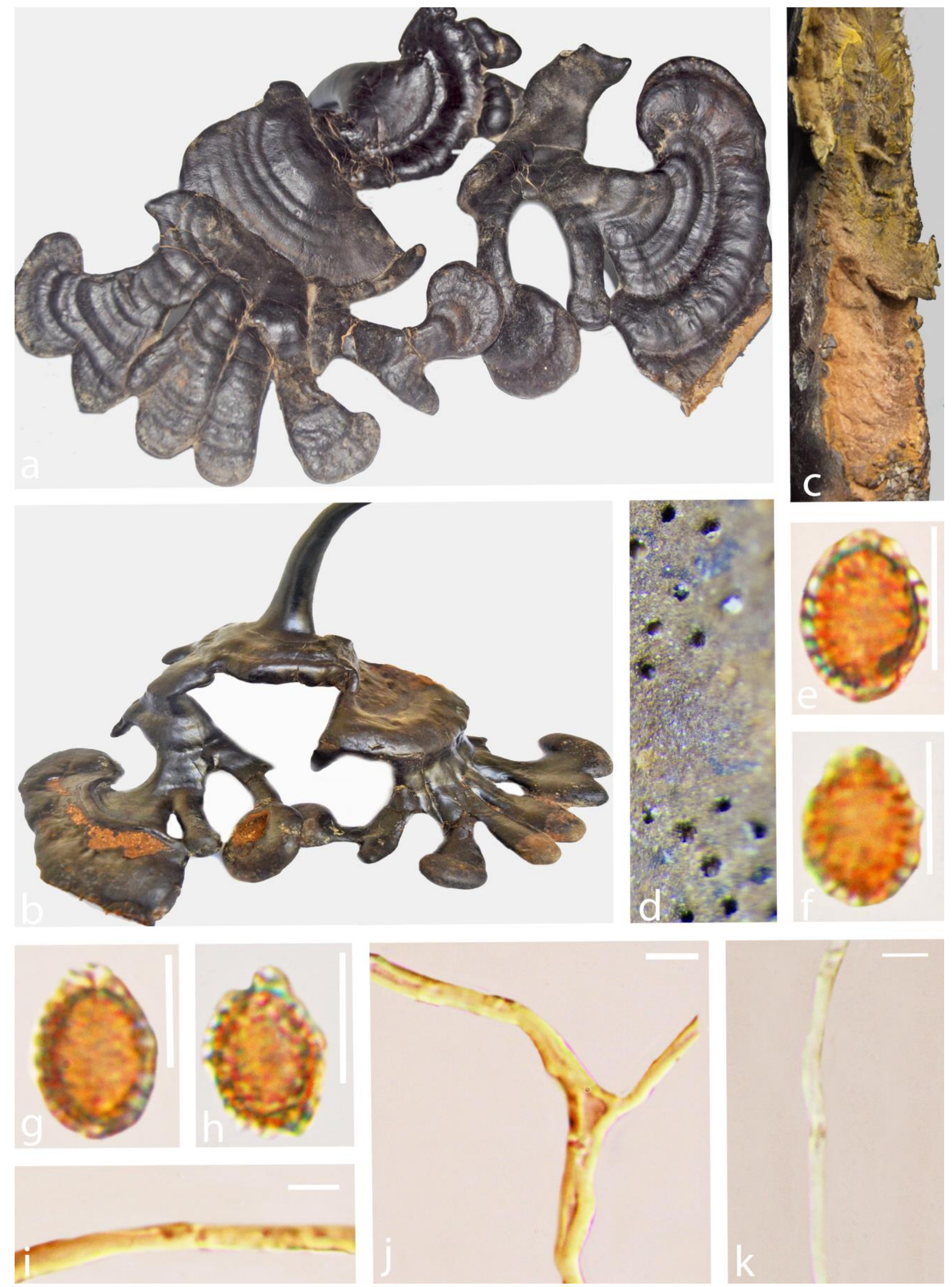

Fig. 7 - Ganoderma sinense specimen no. 6 (GACP17092592). a Upper surface. b Lower surface. c Cut surface. d Pores in the lower surface $(5 \times)$. e-h Spores (100x). i Skeletal hyphae (100x). $\mathrm{j}$ Binding hyphae $(100 \times)$. $\mathrm{k}$ Generative hyphae $(100 \times)$. Scale bars: $\mathrm{e}-\mathrm{h}=10 \mu \mathrm{m}, \mathrm{i}-\mathrm{k}=5 \mu \mathrm{m}$. 

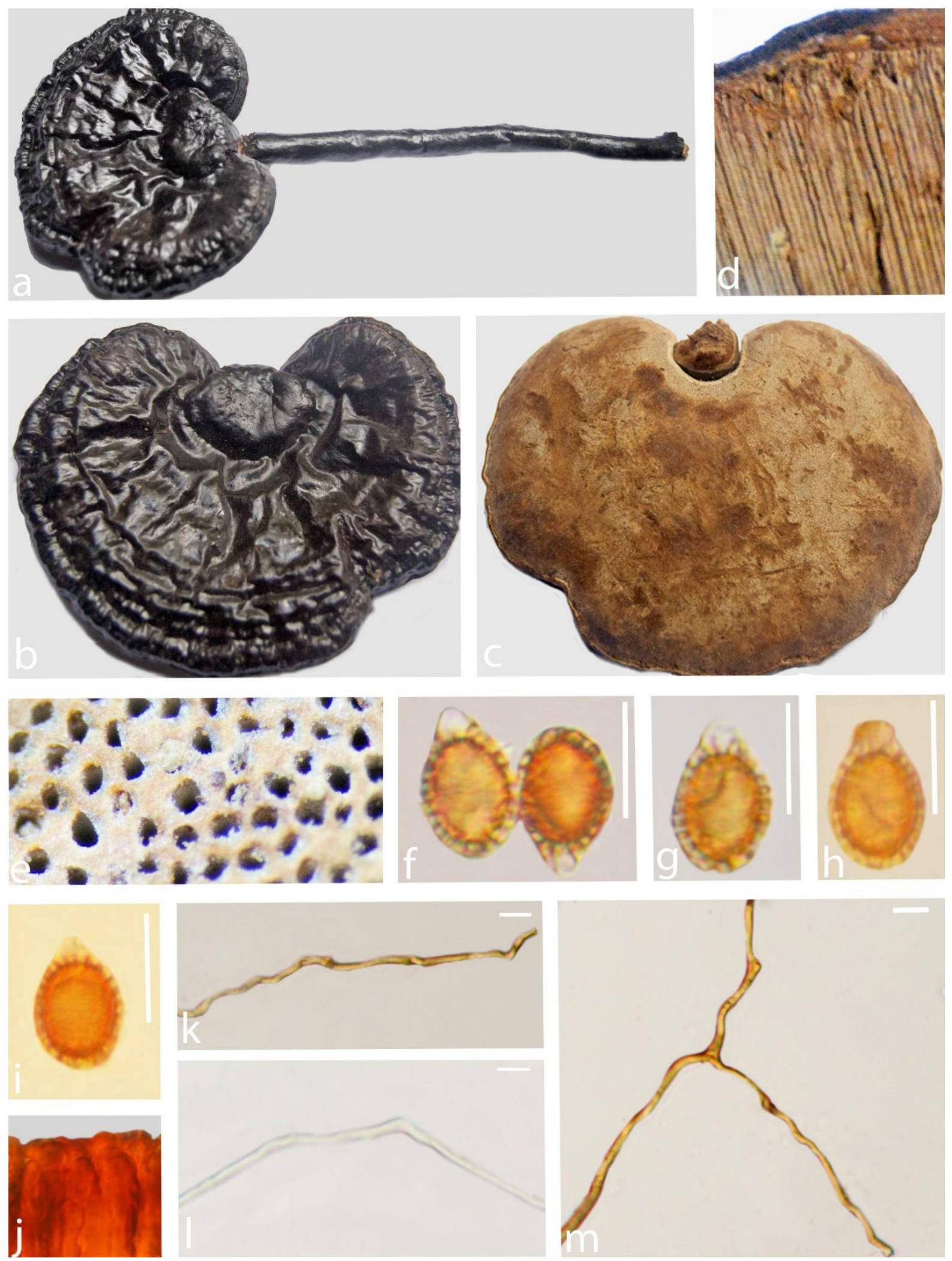

Fig. 8 - Ganoderma sinense specimen no. 7 (GACP171012239). a, b Upper surface. c Lower surface. d Cut surface. e Pores in the lower surface (5x). f-i Spores $(100 \times)$. j Cuticle cells (100x). $\mathrm{k}$ Skeletal hyphae $(100 \times)$. 1 Generative hyphae $(100 \times)$. $\mathrm{m}$ Binding hyphae $(100 \times)$. Scale bars: $\mathrm{f}-\mathrm{j}=$ $10 \mu \mathrm{m}, \mathrm{k}-\mathrm{m}=5 \mu \mathrm{m}$.

\section{Specimen no. 8}

Basidiome annual, stipitate, strongly laccate, corky. Pileus $7-10 \times 6-6.5 \mathrm{~cm}$, up to $1 \mathrm{~cm}$ thick at the base, subreniform; upper surface reddish brown (8E7) to brownish orange (6C8), radially 
rugose, slightly concentrically sulcate, irregularly ruptured crust overlying the context; margin 3 mm thick, blunt, grayish yellow (4B4); lower surface light brown (6D6). Hymenophore up to 15 $\mathrm{mm}$ long, indistinctly stratose; pores initially brown (6D7), bruising dark brown (6F6), pores circular, sub circular or isodiametric, 3-5 per mm. Context up to $1 \mathrm{~cm}$ thick, triplex, dry; upper layer light brown (6D6), fibrous, composed of coarse loose fibrils, corky; middle layer dark brown (6F6), fibrous, corky; lower layer dark brown (6E6), woody. Stipe eccentric, sub cylindrical, concolorous with the pileus, $8 \times 9 \mathrm{~cm}, 1 \mathrm{~cm}$ on the base. Basidiospores $(\mathrm{n}=25)(10.4-) 11.3-12.2-$ 13.1(14.4-) $\times(-6.7) 7.8-8.5-9.2(-9.6) \mu \mathrm{m},\left(Q_{m}=1.4, Q=1.3-1.7\right.$, with myxosporium $) .(\mathrm{n}=25)$ $(8.9-) 9.5-10.2-10.8(-11.7) \times(5.4-) 6.5-7.3-8.1(-8.5) \mu \mathrm{m}\left(Q=1.2-1.9, Q_{m}=1.4\right.$, without myxosporium), ellipsoid, brown (6E6), with a brown (6E6) eusporium bearing thick, short and distinct echinulae, overlaid by a hyaline myxosporium. Pileipellis a hymeniderm, brownish orange (6C8), clavate like cells. Context trimitic; generative hyphae $(\mathrm{n}=20)(0.2-1.2-1.7) \mu \mathrm{m}$ in width, colorless, thin-walled; skeletal hyphae $(\mathrm{n}=20)(2.8-3.5-4.3) \mu \mathrm{m}$ in width, thick walled, nearly solid, sometimes branched, brownish orange (6C6); binding hyphae $(\mathrm{n}=20)(2.4-3.3-4.3) \mu \mathrm{m}$ in width, thick walled, branched, nearly solid, brownish orange (6C6) (Fig. 9).

\section{Specimen no. 9}

Basidiome annual, stipitate, strongly laccate, corky. Pileus $7-8 \times 5-5.5 \mathrm{~cm}$, up to $0.5 \mathrm{~cm}$ thick at the base, reniform, sub-dimidiate; upper surface brownish grey to black (8F8), radially rugose, concentrically sulcate, with irregularly ruptured crust overlying the pellis; margin blunt, concolorous with the pileus; lower surface brown (7E8). Hymenophore up to $15 \mathrm{~mm}$ long, indistinctly stratose; pores initially dark brown (8F8), bruising brown (7E8), pores circular or subcircular, 4-5 per mm. Context up to $1 \mathrm{~cm}$ thick, duplex, dry; lower layer brown (7D8), fibrous, composed of coarse loose fibrils; upper layer dark brown (8F8), corky. Stipe eccentric, sub cylindrical, concolorous with the pileus, $8 \times 13 \mathrm{~cm}$. Basidiospores $(\mathrm{n}=25)(10.6-) 11.5-12.5-$ $13.5(-14.3) \times(7.1-) 7.7-8.3-8.9(-11.7) \mu \mathrm{m}(Q m=1.5, Q=1.1-1.8$ with myxosporium $) .(6.8-) 9.2-$ 10.2-11.1(-11.7) × (5.4-)6.1-6.8-7.7(-8.4) $\mu \mathrm{m}(Q m=1.5, Q=1.1-1.8$, without myxosporium), ellipsoid, brownish orange (7C8) to reddish orange (7B8) with a brown eusporium bearing fine, short, and distinct echinulae, overlaid by a hyaline myxosporium. Pileipellis a hymeniderm, brownish orange (7C8), clavate like cells, dextrinoid. Context dimitic; generative hyphae $(\mathrm{n}=25)$ $(0.5-1.6-3.3) \mu \mathrm{m}$ in width, colorless, thin-walled; skeletal hyphae $(\mathrm{n}=20)(3.3-4.5-5.2) \mu \mathrm{m}$ thickwalled, nearly solid, sometimes branched, orange (5A6); binding hyphae $(\mathrm{n}=20)(3.4-4.6-5.5)$ $\mu \mathrm{m}$, thick-walled, branched, nearly solid, light brown (5A5) (Fig. 10).

\section{Specimen no. 10}

Basidiome annual, stipitate, strongly laccate, branched, corky. Pileus 6-16 ×9-11 cm, up to $1.0 \mathrm{~cm}$ thick at the base, suborbicular; upper surface dark brown (8F8) to brownish black (6C8), slightly concentrically sulcate, radially rugose, irregularly ruptured crust overlying the context; margin blunt, yellow brown (5D8); lower surface brown (7E8). Hymenophore up to $25 \mathrm{~mm}$ long, indistinctly stratose; pores initially dark brown (8F8), bruising brown (7E8), pores circular or sub circular, 3-5 per mm. Context up to $1 \mathrm{~cm}$ thick, duplex, dry, lower layer brown (7D8), fibrous, composed of coarse loose fibrils; upper layer dark brown (8F8), corky. Stipe eccentric, sub cylindrical, concolorous with the pileus, $5 \times 7 \mathrm{~cm}, 1.5 \mathrm{~cm}$ at the base. Basidiospores $(\mathrm{n}=25)(9.5-$ )10.8-12.2-13.4(13.7-) $\times(-6.6) 7.5-8.2-8.9(-9.1) \mu \mathrm{m}\left(Q_{m}=1.5, Q=1.2-1.7\right.$, with myxosporium). (7.5-)9.3-10.4-11.5(-12) $\times(5.4-) 6-6.8-7.7(-7.9) \mu \mathrm{m} \quad\left(Q_{m}=1.5, Q=1.2-1.9\right.$, without myxosporium), ellipsoid, brownish orange (7C8) to reddish orange (7B8) with a brown (7D8), eusporium bearing fine, short and distinct echinulae, overlaid by a hyaline myxosporium. Pileipellis a hymeniderm, brownish orange (7C8), clavate like cells. Context trimitic, generative hyphae $(\mathrm{n}=20)(1.1-2-2.9) \mu \mathrm{m}$, colorless, thin-walled; skeletal hyphae $(\mathrm{n}=25)(3.3-3.9-5.7) \mu \mathrm{m}$, thick walled, nearly solid, sometimes branched, orange (5A6); binding hyphae $(\mathrm{n}=20)(2.5-3.9-$ 5.1) $\mu \mathrm{m}$, thick walled, branched, nearly solid, orange (5A6) (Fig. 11). 

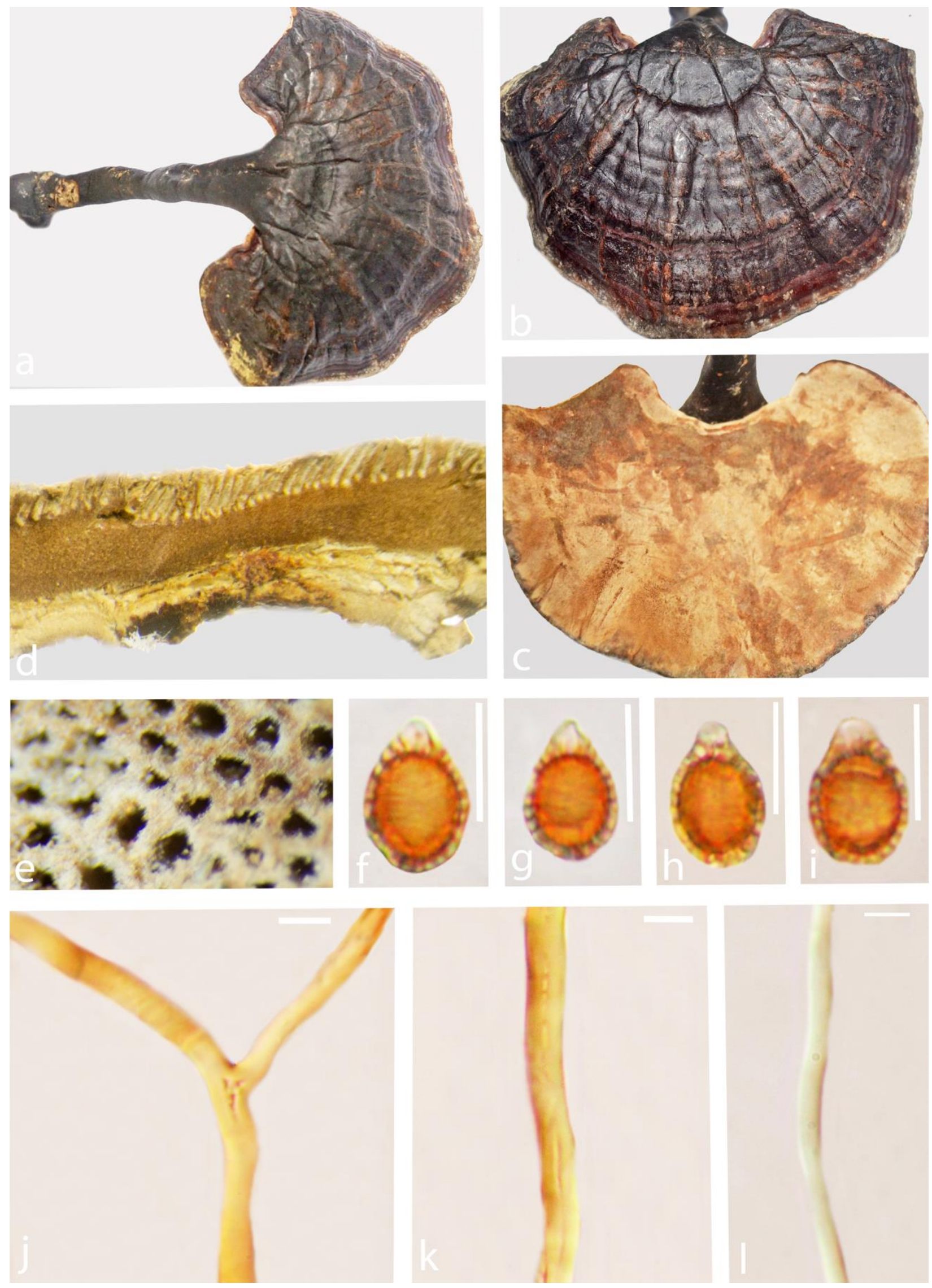

Fig. 9 - Ganoderma sinense specimen no. 8 (GACP17092588). a, b Upper surface. c Lower surface. d Cut surface. e Pores in the lower surface $(5 \times)$. f-i Spores $(100 \times)$. j Binding hyphae (100x). k Skeletal hyphae (100x). 1 Generative hyphae (100x). Scale bars: $f-i=10 \mu \mathrm{m}, \mathrm{j}-1=5 \mu \mathrm{m}$. 

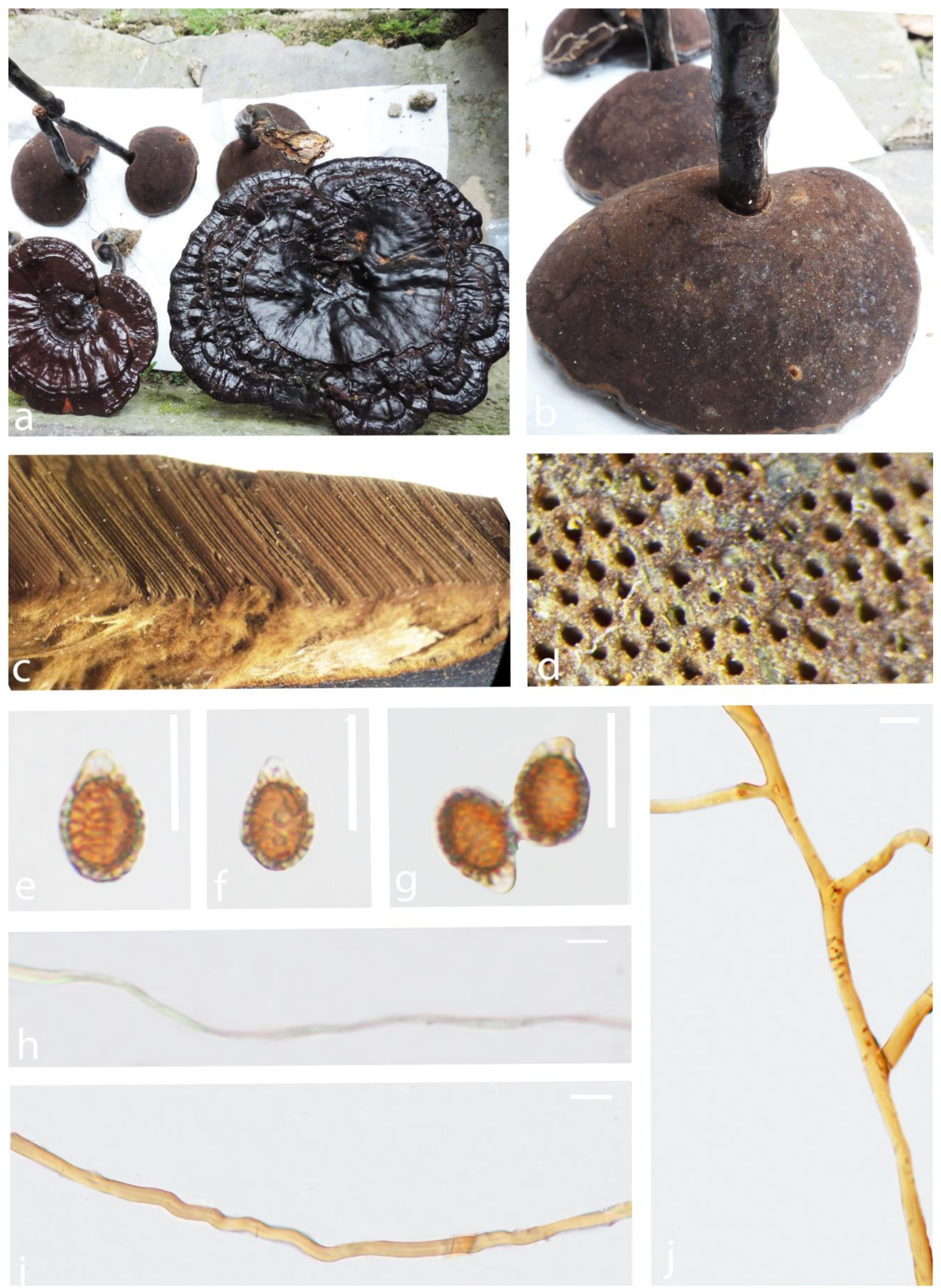

Fig. 10 - Ganoderma sinense specimen no. 9 (GACP14081236). a Upper surface. b Lower surface.

c Cut surface. d Pores in the lower surface (5x). e-g Spores (100x). h Generative hyphae (100x). i Skeletal hyphae $(100 \times)$. j Binding hyphae $(100 \times)$. Scale bars: $e-g=10 \mu \mathrm{m}, \mathrm{h}-\mathrm{j}=5 \mu \mathrm{m}$. 

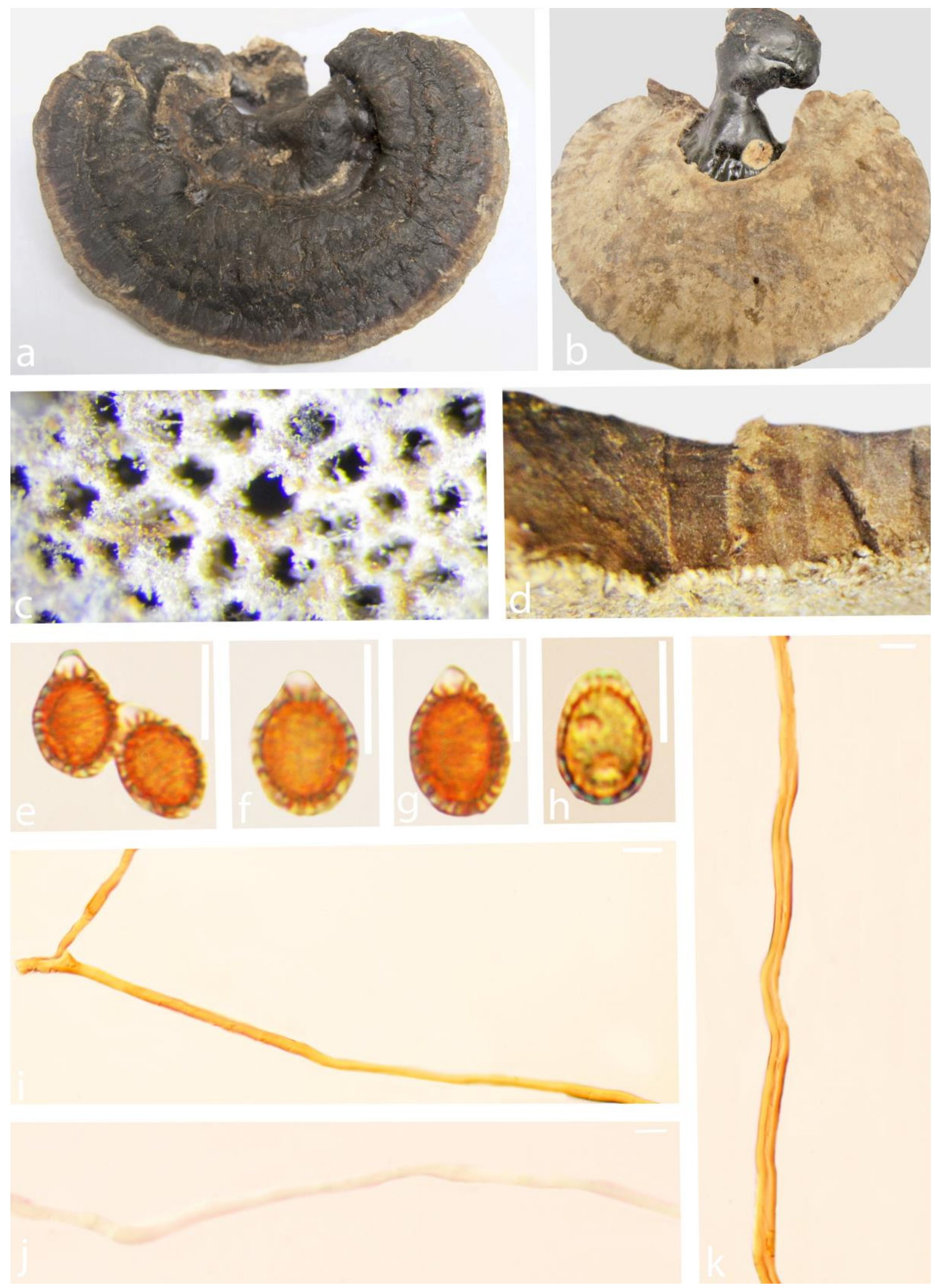

Fig. 11 - Ganoderma sinense specimen no. 10 (GACP17092548). a Upper surface. b Lower surface. c Pores in the lower surface $(5 x)$. d Cut surface. e-h Spores $(100 x)$. i Binding hyphae $(100 \times)$. j Generative hyphae $(100 \times)$. k Skeletal hyphae $(100 \times)$. Scale bars: $\mathrm{e}-\mathrm{h}=10 \mu \mathrm{m}, \mathrm{i}-\mathrm{k}=5$ $\mu \mathrm{m}$.

\section{Specimen no. 11}

Basidiome annual, stipitate, laccate, corky. Pileus $2.5-4 \times 1.5-2.5 \mathrm{~cm}$, up to $0.5 \mathrm{~cm}$ thick at the base, sub orbicular to sub reniform; upper surface brownish black (6C8), radially rugose with 
turberculate bumps and ridges, slightly concentrically sulcate, irregularly ruptured crust overlying the context; margin $2 \mathrm{~mm}$ thick, soft, concolorous with the pileus; lower surface light brown (6D6). Hymenophore up to $12 \mathrm{~mm}$ long, indistinctly stratose; pores brownish grey (6D2), pores circular or sub circular, 3-5 per mm. Context up to $4 \mathrm{~mm}$ thick, duplex, dry; upper layer dark brown (7F8); lower layer pale brown (5A5), fibrous, composed of coarse loose fibrils; woody. Stipe eccentric, sub cylindrical, concolorous with the pileus, $3 \times 8 \mathrm{~cm}, 1 \mathrm{~cm}$ at the base. Basidiospores $(\mathrm{n}=20)$ (12.6-)13.1-13.8-14.5(13.6-) × (-7.8)8.5-9.1-9.7(-10.3) $\mu \mathrm{m}\left(Q_{m}=1.5, Q=1.4-1.8\right.$, with myxosporium). (10.5-)11.3-12.0-12.7(-13.4) × (6.1-)6.7-7.4-8.1(-8.5) $\mu \mathrm{m}\left(Q_{m}=1.6, Q=1.4-\right.$ 2.0, without myxosporium), ellipsoid, brown (6E4), with a pale brown (5A5) eusporium bearing fine, short and distinct echinulae, overlaid by a hyaline myxosporium. Pileipellis a hymeniderm, brownish orange $(6 \mathrm{C} 4)$, clavate like cells. Context trimitic; generative hyphae $(\mathrm{n}=20)(0.8-1.2-$ 1.6) $\mu \mathrm{m}$ in width, colourless, thin walled without clamp connections; skeletal hyphae $(\mathrm{n}=20)(3.4-$ 4.6-5.2) $\mu \mathrm{m}$ in width, thick walled, nearly solid, sometimes branched, brown (6E4); binding hyphae $(\mathrm{n}=20)(3.4-4.2-5.9) \mu \mathrm{m}$ in width, thick walled, branched, nearly solid, brown (6E4) (Fig. 8) (Fig. 12).

\section{Specimen no. 12}

Basidiome annual, stipitate, laccate, corky. Pileus $1.5-4.5 \times 1-2.5 \mathrm{~cm}$, up to $0.5 \mathrm{~cm}$ thick at the base, subreniform to sub orbicular; upper surface brownish black (6C8), radially rugose with turberculate bumps and ridges, distinctly concentrically sulcate, irregularly ruptured crust overlying the context, swollen at the point of attachment; margin $2 \mathrm{~mm}$ thick, soft, concolourous with the pileus; lower surface light brown (6D6). Hymenophore up to $12 \mathrm{~mm}$ long, indistinctly stratose; pores initially brownish grey (6D2), bruising dark brown (6F6), pores sub circular or isodiametric, 2-4 per mm. Context up to $3 \mathrm{~mm}$ thick, duplex, dry; upper layer dark brown ((7F8); lower layer light brown (6D6), fibrous, composed of coarse loose fibrils, woody. Stipe eccentric, sub cylindrical, concolorous with the pileus, $4 \times 8 \mathrm{~cm}, 0.5 \mathrm{~cm}$ at the base. Basidiospores $(\mathrm{n}=20)(10.1-$ )11.4-12.5-13.5(14.4-) × (-6.9)7.8-8.5-9.1(-9.7) $\mu \mathrm{m}\left(Q_{m}=1.4, Q=1.2-1.7\right.$, with myxosporium). $(8.5-) 9.7-10.8-11.9(-12.8) \times(5.1-) 6-6.8-7.6(-8.2) \mu \mathrm{m} \quad\left(Q_{m}=1.6, Q=1.2-2\right.$, without myxosporium), ellipsoid, brown (6E4), with a pale brown (5A5), eusporium bearing fine, short and distinct echinulae, overlaid by a hyaline myxosporium. Pileipellis a hymeniderm, brownish orange (6C4), clavate like cells. Context dimitic; generative hyphae not observed; skeletal hyphae $(\mathrm{n}=20)$ (2.5-3.3-4.2) $\mu \mathrm{m}$ in width, thick walled, nearly solid, sometimes branched, pale brown (5A5); binding hyphae $(\mathrm{n}=20)(2.6-3.3-4.1) \mu \mathrm{m}$ in width, thick walled, branched, nearly solid, brownish orange (6C4) (Fig. 13).

\section{Specimen no. 13}

Basidiome annual, stipitate, laccate, corky. Pileus $2.5-5 \times 1-2.5 \mathrm{~cm}$, up to $0.4 \mathrm{~cm}$ thick at the base, reniform; upper surface brownish black (6C8), slightly concentric, not sulcate, radially rugose with turberculate bumps and ridges, irregularly ruptured crust overlying the context; margin $2 \mathrm{~mm}$ thick, soft, concolorous with the pileus; lower surface light brown (6D6). Hymenophore up to 12 $\mathrm{mm}$ long, indistinctly stratose; pores initially brownish grey (6D2), bruising dark brown (6F6), pores circular, sub circular or isodiametric, 3-5 per mm. Context up to $8 \mathrm{~mm}$ thick, triplex, dry; upper layer dark brown ((7F8); middle layer light brown (6D6); lower layer brown (6E4) to dark brown (7F8), fibrous, composed of coarse loose fibrils, woody. Stipe eccentric, sub cylindrical, concolorous with the pileus, $3.5 \times 8 \mathrm{~cm}, 1 \mathrm{~cm}$ thick at the base. Basidiospores $(\mathrm{n}=20)(10.5-$ )11.8-12.9-13.9(14.3-) $\times(-8.1) 8.3-8.9-9.5(-10) \mu \mathrm{m}\left(Q_{m}=1.4, Q=1.2-1.7\right.$, with myxosporium). $(8.8-) 10.1-11.2-12.3(-12.9) \times(5.5-) 6.5-7.3-8.1(-8.4) \mu \mathrm{m}\left(Q_{m}=1.5, Q=1.2-1.9\right.$, without myxosporium), ellipsoid, brown (6E4), with a pale brown (5A5) bearing fine, short and distinct echinulae, overlaid by a hyaline myxosporium. Pileipellis a hymeniderm, brownish orange (6C4), clavate like cells. Context trimitic; generative hyphae $(\mathrm{n}=20)(0.4-1.1-1.5) \mu \mathrm{m}$ in width, colourless, thin walled without clamp connections; skeletal hyphae $(\mathrm{n}=20)(4-4.8-5.9) \mu \mathrm{m}$ in 
width, thick walled, nearly solid, sometimes branched, brownish orange (6C4); binding hyphae ( $\mathrm{n}=$ 20) (1.2-2.6-3.9) $\mu \mathrm{m}$ in width, thick walled, branched, nearly solid, pale brown (5A5) (Fig. 14).

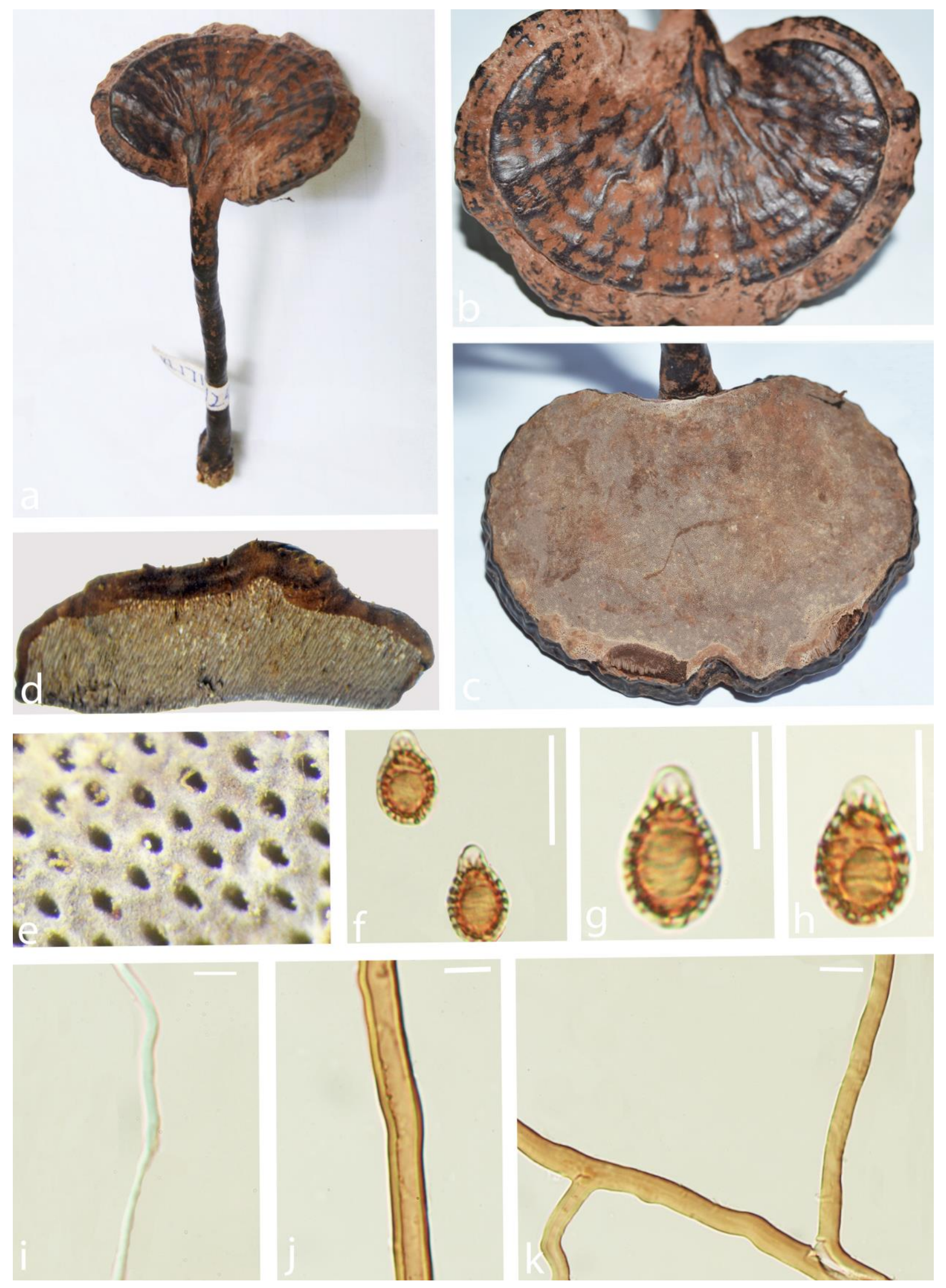

Fig. 12 - Ganoderma sinense specimen no. 11 (GACP17101242). a, b Upper surface. c Lower surface. d Cut surface. e Pores in the lower surface $(5 \times)$. $\mathrm{f}-\mathrm{h}$ Spores $(100 \times)$. i Generative hyphae $(100 \times)$. j Skeletal hyphae (100x). k Binding hyphae $(100 \times)$. Scale bars: $\mathrm{f}-\mathrm{h}=10 \mu \mathrm{m}, \mathrm{i}-\mathrm{l}=5 \mu \mathrm{m}$. 

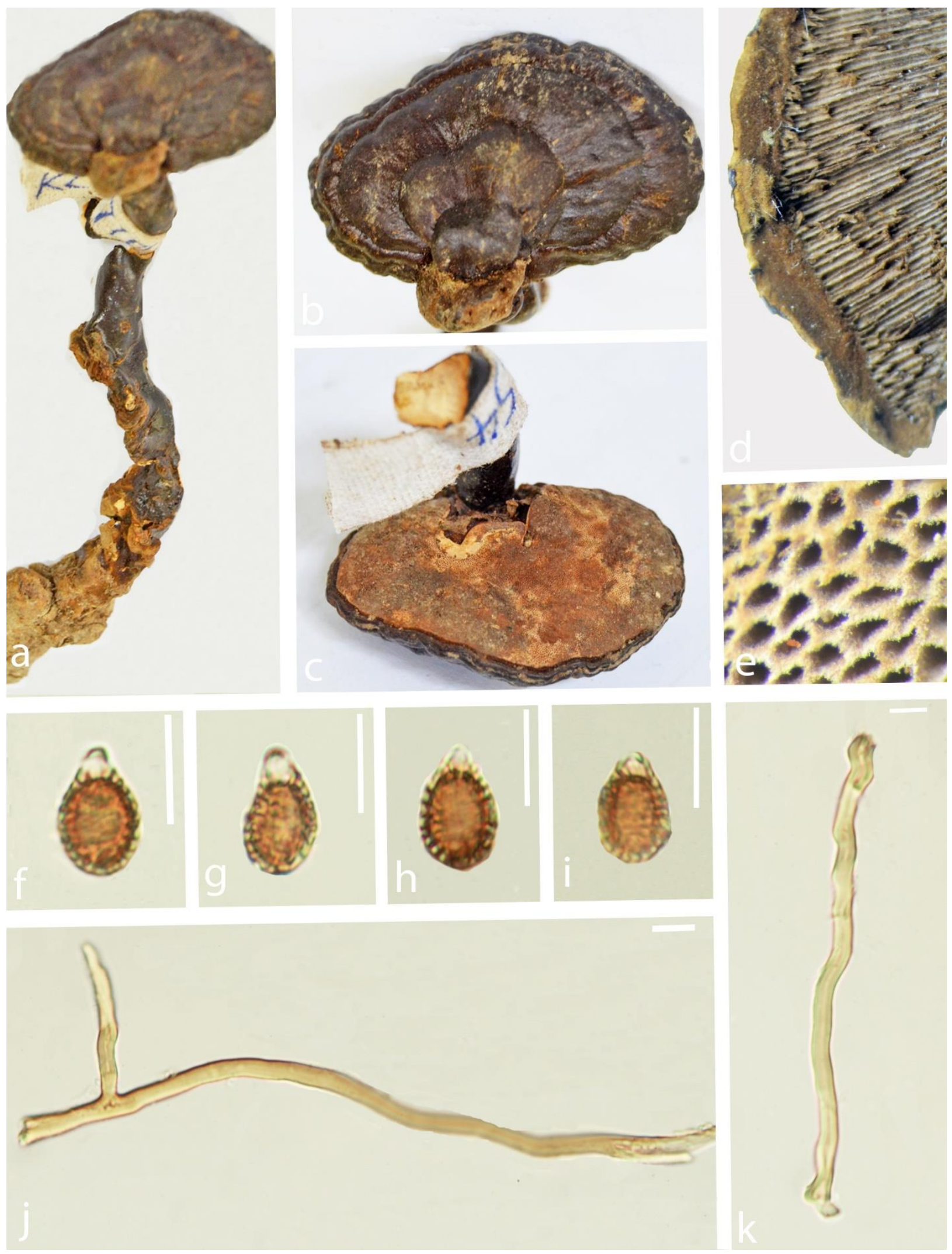

Fig. 13 - Ganoderma sinense specimen no. 12 (GACP17101254). a, b Upper surface. c Lower surface. d Cut surface. e Pores in the lower surface $(5 \times)$. f-i Spores $(100 \times)$. j Binding hyphae $(100 \times)$. k Skeletal hyphae $(100 \times)$. Scale bars: $\mathrm{f}-\mathrm{i}=10 \mu \mathrm{m}, \mathrm{j}-\mathrm{k}=5 \mu \mathrm{m}$.

\section{Specimen no. 14}

Basidiome annual, stipitate, laccate, corky. Pileus $2-4 \times 1-1.5 \mathrm{~cm}$, up to $0.5 \mathrm{~cm}$ thick at the base, flabelliform; upper surface reddish brown (8E7) to brownish black (6C8), radially rugose 
with turberculate bumps and ridges, irregularly ruptured crust overlying the context; margin $2 \mathrm{~mm}$ thick, soft, yellowish brown (5D8); lower surface light brown (6D6). Hymenophore up to $12 \mathrm{~mm}$ long, indistinctly stratose; pores initially brownish grey (6D2), bruising dark brown (6F6), pores circular, 3-4 per mm. Context up to $1 \mathrm{~cm}$ thick, duplex, dry; upper layer dark brown (7F8), lower layer brown (6E4) to dark brown ((7F8), fibrous, composed of coarse loose fibrils, woody. Stipe eccentric, sub cylindrical, concolorous with the pileus, $5.5 \times 10 \mathrm{~cm}, 1 \mathrm{~cm}$ thick at the base. Basidiospores $(\mathrm{n}=20)(10.3-) 11.5-12.8-14.2(15.6-) \times(-8.1) 8.5-9.2-9.8(-10.9) \mu \mathrm{m}\left(Q_{m}=1.4, Q\right.$ $=1.1-1.7$, with myxosporium). (8.3-)9.7-11.1-12.5(-13.8) × (6.4-)6.9-7.7-8.5(-10.1) $\mu \mathrm{m}\left(Q_{m}=\right.$ $1.4, Q=1.1-1.8$, without myxosporium), ellipsoid, brown (6E4), with a pale brown (5A5) eusporium bearing fine, short and distinct echinulae, overlaid by a hyaline myxosporium. Pileipellis a hymeniderm, brownish orange (6C4), clavate like cells, dextrinoid. Context trimitic; generative hyphae $(\mathrm{n}=20)(0.4-1-1.5) \mu \mathrm{m}$ in width, colourless, thin walled without clamp connections; skeletal hyphae $(\mathrm{n}=20)(2.1-3.9-3.9) \mu \mathrm{m}$ in width, thick walled, nearly solid, sometimes branched, brown (6E4) to brownish orange $(6 \mathrm{C} 4)$; binding hyphae $(\mathrm{n}=20)(1.4-2.7-$ 3.7) $\mu \mathrm{m}$ in width, thick walled, branched, nearly solid, pale brown (5A5) (Fig. 15).

\section{Specimen no. 15}

Basidiome annual, stipitate, laccate, corky. Pileus $8-12 \times 7-9 \mathrm{~cm}$, up to $1.5 \mathrm{~cm}$ thick at the base, spathulate; upper surface dark brown $(8 \mathrm{~F} 8)$ to brownish black $(6 \mathrm{C} 8)$, radially rugose with turberculate bumps and ridges and rivulose depressions, concentrically sulcate zones, irregularly ruptured crust overlying the context; margin blunt to truncate, concolorous with the pileus; lower surface brown (7E8). Hymenophore up to $15 \mathrm{~mm}$ long, indistinctly stratose; pores initially dark brown (8F8), bruising brown (7E8), pores circular, sub circular or isodiametric, 3-5 per mm. Context up to $8 \mathrm{~mm}$ thick, duplex, dry, lower layer brown (7D8), fibrous, composed of coarse loose fibrils; upper layer dark brown (8F8), corky. Stipe eccentric, sub cylindrical, concolorous with the pileus, $6 \times 11 \mathrm{~cm}, 2.5 \mathrm{~cm}$ thick at the base. Basidiospores $(\mathrm{n}=25)(9.8-) 10.6-11.3-11.9(12.1-) \times$ (-6.9)7.3-8-8.6(-9.3) $\mu \mathrm{m}\left(Q_{m}=1.4, Q=1.2-1.6\right.$, with myxosporium). (7.6-)8.2-8.8-9.5(-10.3) $\times$ (4.3-)5.3-6.1-6.9(-7.6) $\mu \mathrm{m}\left(Q_{m}=1.5, Q=1.1-1.9\right.$, without myxosporium), ellipsoid, brownish orange (7C8) to reddish orange (7B8) with a brown (7D8) eusporium bearing fine, short and distinct echinulae, overlaid by a hyaline myxosporium. Pileipellis a hymeniderm, brownish orange (7C8), clavate like cells. Context trimitic; generative hyphae $(\mathrm{n}=20)(0.6-1.4-2.4) \mu \mathrm{m}$, hyaline, colourless, thin walled without clamp connections; skeletal hyphae $(\mathrm{n}=20)(0.9-2.1-3.5) \mu \mathrm{m}$, thick walled, nearly solid, sometimes branched; binding hyphae $(\mathrm{n}=20)(2.7-3.8-5.3) \mu \mathrm{m}$, thick walled, branched, nearly solid, orange (5A6) (Fig. 16).

\section{Specimen no. 16}

Basidiome annual, stipitate, strongly laccate, corky. Pileus $4-5.5 \times 3-4 \mathrm{~cm}$, up to $1 \mathrm{~cm}$ thick at the base, spathulate; upper surface dark brown (8F8) to brownish black (6C8), radially rugose, irregularly ruptured crust overlying the context; margin blunt, concolorous with the pileus; lower surface brown (7E8). Hymenophore up to $15 \mathrm{~mm}$ long, indistinctly stratose; pores initially dark brown (8F8), circular, sub circular or isodiametric, 3-5 per mm. Context up to $1 \mathrm{~cm}$ thick, duplex, dry; lower layer brown (7D8), fibrous, composed of coarse loose fibrils; upper layer dark brown (8F8), corky. Stipe eccentric, sub cylindrical, concolorous with the pileus, $8 \times 13 \mathrm{~cm}, 1 \mathrm{~cm}$ at the base. Basidiospores $(\mathrm{n}=20)(9.9-) 10.5-11.3-12.1(12.8-) \times(-6.6) 7.5-8.2-8.6(-9.1) \mu \mathrm{m}\left(Q_{m}=\right.$ $1.4, Q=1.2-1.5$, with myxosporium). (8.1-)8.7-9.4-10(-10.4) $\times(5.8-) 6.3-6.7-7.3(-7.6) \mu \mathrm{m}\left(Q_{m}\right.$ $=1.4, Q=1.2-1.6$, without myxosporium), ellipsoid, brown, with a brown eusporium bearing fine, short and distinct echinulae, overlaid by a hyaline myxosporium. Pileipellis a hymeniderm, orange (5A6), clavate like cells, dextrinoid. Context trimitic; generative hyphae $(\mathrm{n}=20)(0.5-1-1.5) \mu \mathrm{m}$ in width, colorless, thin-walled; skeletal hyphae $(\mathrm{n}=20)(2.2-3.4-5.4) \mu \mathrm{m}$ thick walled, nearly solid, sometimes branched, orange (5A6); binding hyphae $(\mathrm{n}=20)(1.1-3.3-5.2) \mu \mathrm{m}$ in width, thick walled, branched, nearly solid, orange (5A6). (Fig. 17). 

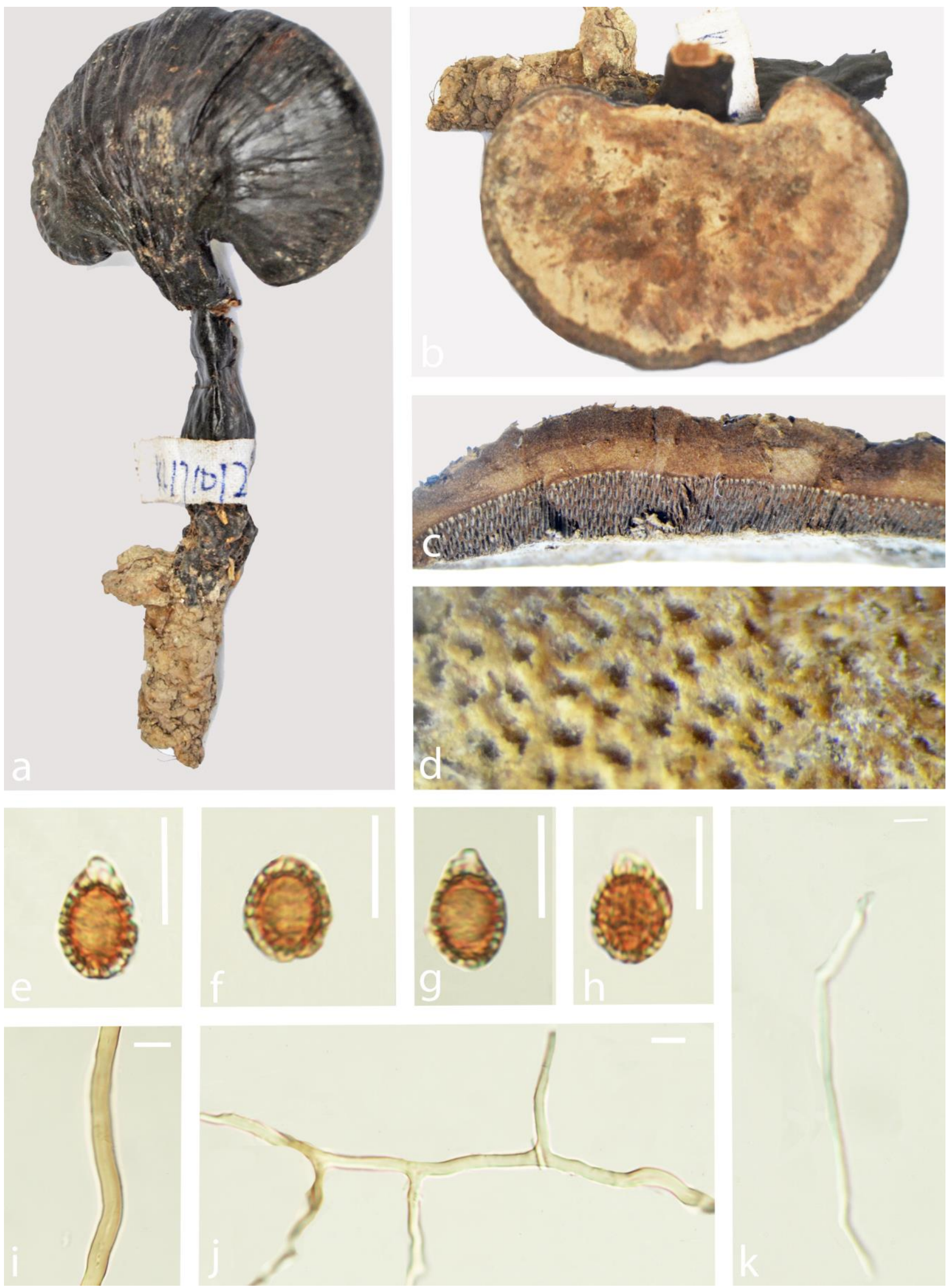

Fig. 14 - Ganoderma sinense specimen no. 13 (GACP17101260). a Upper surface. b Lower surface. c Cut surface. d Pores in the lower surface (5x). e-h Spores (100x). i Skeletal hyphae $(100 \times)$. j Binding hyphae $(100 \times)$. $k$ Generative hyphae $(100 \times)$. Scale bars: $\mathrm{e}-\mathrm{h}=10 \mu \mathrm{m}, \mathrm{i}-\mathrm{k}=5$ $\mu \mathrm{m}$. 

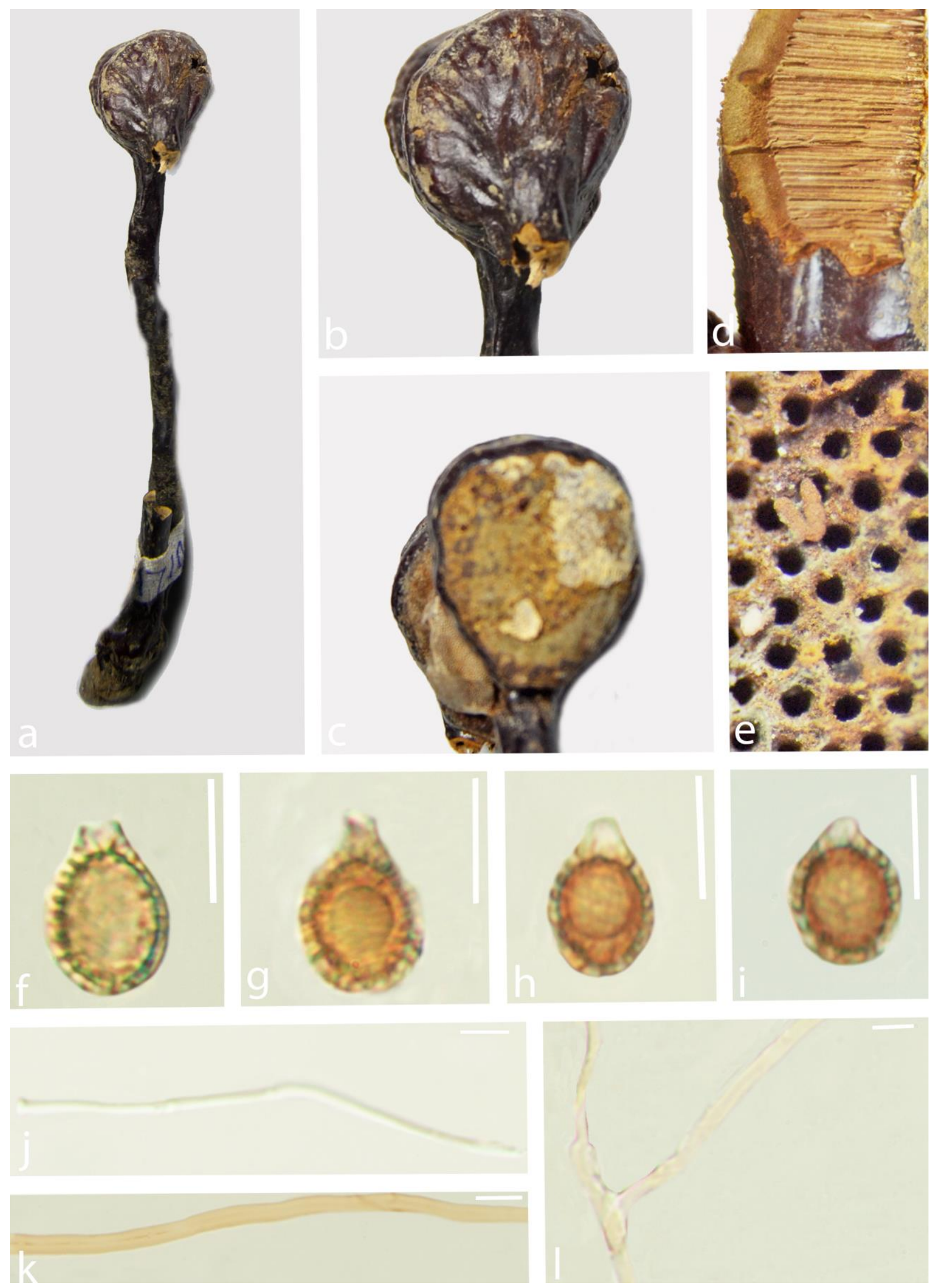

Fig. 15 - Ganoderma sinense specimen no. 14 (GACP17101270). a, b Upper surface. c Lower surface. d Cut surface. e Pores in the lower surface (5x). f-i Spores (100x). j Generative hyphae (100x). k Skeletal hyphae (100x). 1 Binding hyphae (100x). Scale bars: $f-I=10 \mu \mathrm{m}, j-1=5 \mu \mathrm{m}$. 

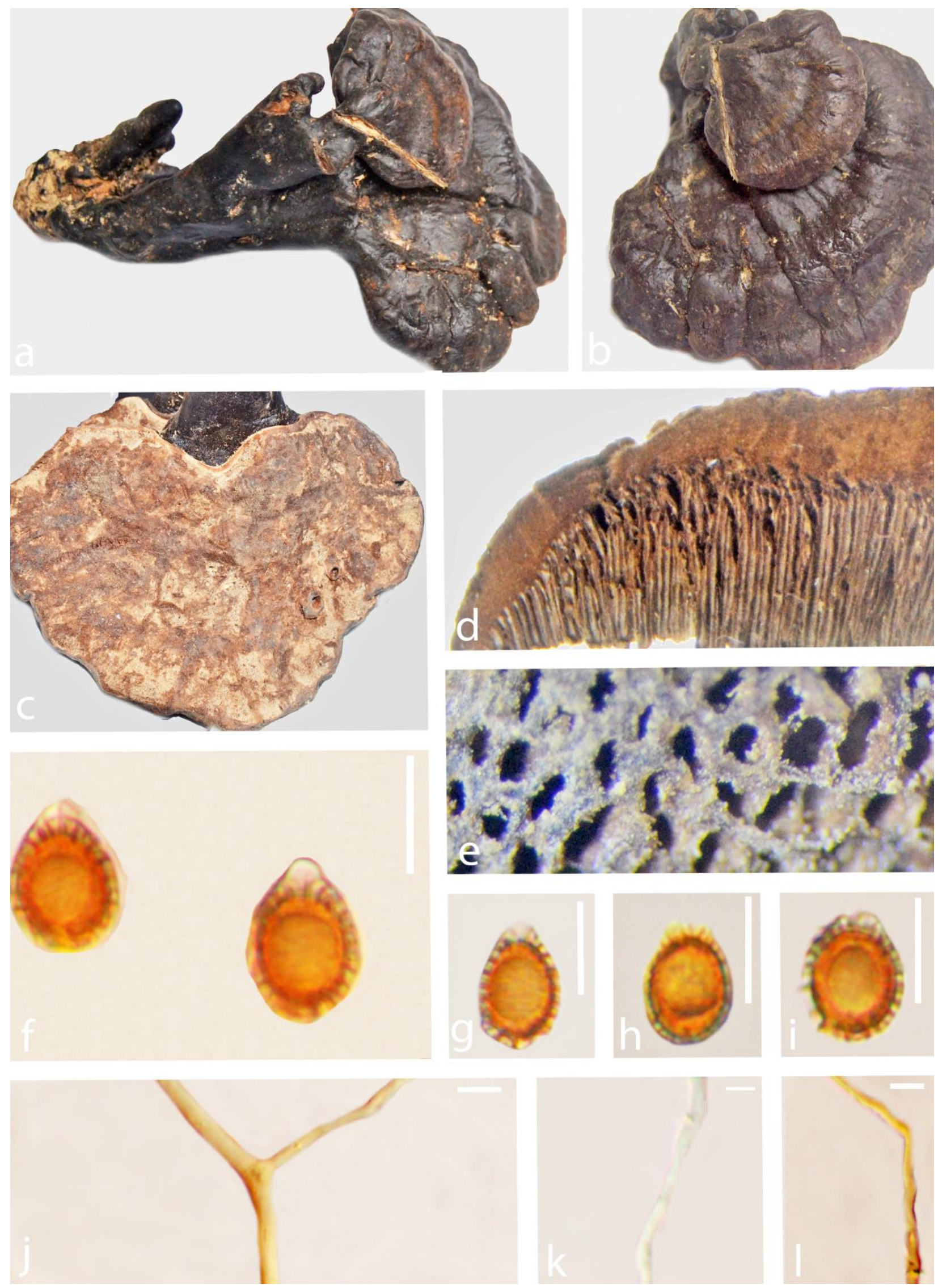

Fig. 16 - Ganoderma sinense specimen no. 15 (GACP17092581). a, b Upper surface. c Lower surface. d Cut surface. e Pores in the lower surface $(5 \times)$. $\mathrm{f}-\mathrm{i}$ Spores $(100 \times)$ ). i Binding hyphae (100x). j Generative hyphae (100x). k Skeletal hyphae (100x). Scale bars: $f-i=10 \mu \mathrm{m}, \mathrm{j}-1=5 \mu \mathrm{m}$. 

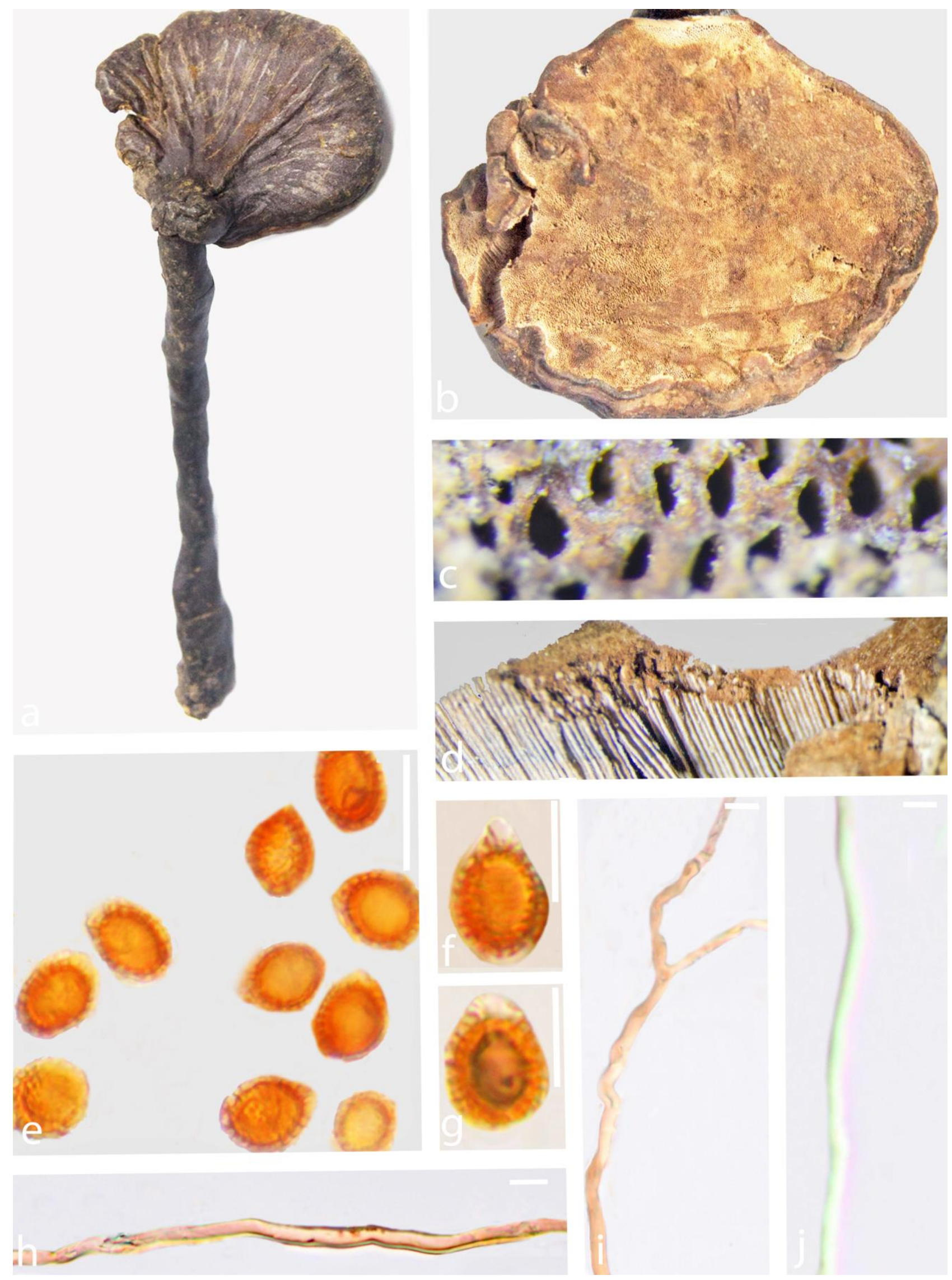

Fig. 17 - Ganoderma sinense specimen no. 16 (GACP17092520). a Upper surface. b Lower surface. c Cut surface $(5 \times)$. d Pores in the lower surface. e-g spores $(100 \times)$. h Generative hyphae (40x), i Binding hyphae (40x). j Skeletal hyphae (40x). Scale bars: $\mathrm{e}-\mathrm{g}=10 \mu \mathrm{m}, \mathrm{h}-\mathrm{j}=5 \mu \mathrm{m}$. 


\section{Specimen no. 17}

Basidiome annual, stipitate, laccate, corky. Pileus $7.5-11 \times 6-7.5 \mathrm{~cm}$, up to $1.5 \mathrm{~cm}$ thick at the base, reniform; upper surface dark brown (8F8) to brownish black (6C8), radially rugose, concentrically sulcate zones, irregularly ruptured crust overlying the context; margin blunt or wavy, concolorous with the pileus, lower surface brown (7E8). Hymenophore up to $20 \mathrm{~mm}$ long, indistinctly stratose; pores initially dark brown (8F8), bruising brown (7E8) pores circular, sub circular or isodiametric, 4-5 per $\mathrm{mm}$. Context up to $5 \mathrm{~mm}$ thick, duplex, dry, lower layer brown (7D8), fibrous, composed of coarse loose fibrils; upper layer dark brown (8F8), corky. Stipe eccentrically stipitate, sub cylindrical, concolorous with the pileus, $5 \times 7 \mathrm{~cm}, 1.5 \mathrm{~cm}$ at the base. Basidiospores $(\mathrm{n}=25)(8.5-) 9.1-10.2-11.3(12.3-) \times(-4.7) 6.2-7-7.8(-8.3) \mu \mathrm{m}\left(Q_{m}=1.5, Q=1.1-\right.$ 1.9 , with myxosporium). (6.2-)6.6-7.5-8.3(-8.9) $\times(3.2-) 4.2-5-5.7(-6.2) \mu \mathrm{m}\left(Q_{m}=1.5, Q=2.1-\right.$ 1.2 , without myxosporium), ellipsoid, brownish orange (7C8) to reddish orange (7B8) with a brown (7D8) eusporium bearing fine, short and distinct echinulae overlaid by a hyaline myxosporium. Pileipellis a hymeniderm, orange (5A6), clavate like cells, dextrinoid. Context trimitic; generative hyphae $(\mathrm{n}=30)(0.8-1.3-1.8) \mu \mathrm{m}$ in width, colourless, thin walled without clamp connections; skeletal hyphae $(\mathrm{n}=30)(2.6-4.1-5.8) \mu \mathrm{m}$ in width, thick walled, nearly solid, sometimes branched, orange $(5 \mathrm{~A} 6)$; binding hyphae $(\mathrm{n}=20)(1.3-1.7-2.4) \mu \mathrm{m}$ in width, thick walled, branched, nearly solid, orange (5A6) (Fig. 18).

\section{Specimen no. 18}

Basidiome annual, stipitate, strongly laccate, corky. Pileus $7-10 \times 6-8 \mathrm{~cm}$, up to $1 \mathrm{~cm}$ thick at the base, subreniform; upper surface brown (6E4) to brownish black (6C8), yellowish brown (5D8) at the margin, slightly concentrically sulcate zones, radially rugose, irregularly ruptured crust overlying the context; margin $2 \mathrm{~mm}$ thick, blunt, yellow brown (5D8); lower surface pale brown (5A5). Hymenophore up to $10 \mathrm{~mm}$ long, indistinctly stratose; pores initially brownish grey (6D2), pores circular, sub circular or isodiametric, 3-5 per mm. Context up to $1 \mathrm{~cm}$ thick, duplex, dry, upper layer dark brown (7F8), corky; lower layer pale brown (5A5), fibrous, composed of coarse loose fibrils, woody. Stipe eccentric, sub cylindrical, concolorous with the pileus, $5 \times 8 \mathrm{~cm}, 1.5 \mathrm{~cm}$ at the base. Basidiospores $(\mathrm{n}=20)(9.6-) 10.3-11.4-12.6(13.5-) \times(-6.7) 7.3-7.8-8.4(-8.9) \mu \mathrm{m},\left(Q_{m}\right.$ $=1.5, Q=1.1-1.7$, with myxosporium). (7.8-)8.4-9.4-10.4 (-11.5) $\times(4.6-) 5.6-6.2-6.8(-7.4) \mu \mathrm{m}$ $\left(Q_{m}=1.5 . Q=1.2-1.8\right.$, without myxosporium), ellipsoid, brown (6E4), with a pale brown (5A5) eusporium bearing fine, short and distinct echinulae, overlaid by a hyaline myxosporium. Pileipellis a hymeniderm, brown (5D8), clavate like cells. Context trimitic, generative hyphae $(\mathrm{n}=$ 20) $(0.6-1.3-1.8) \mu \mathrm{m}$ in width, colorless, thin-walled; skeletal hyphae $(\mathrm{n}=20)(3.8-5.2-6.5) \mu \mathrm{m}$ in width, thick walled, nearly solid, sometimes branched, pale brown (5A5); binding hyphae $(\mathrm{n}=20)$ (2.2-3.2-4.4) $\mu \mathrm{m}$ in width, thick walled, branched, nearly solid, brown (6E4) (Fig. 19).

Notes - Ganoderma sinense was originally described from Hainan Province, China by Zhao et al. (1979) and is characterized by slightly longitudinally crested basidiospores and a uniformly brown to dark brown context. Although the holotype of this species is now very scanty and difficult to observe, our $G$. sinense collections agree with that of the holotype described by Wang \& Wu (2007), and with descriptions given by Bi et al. (1993), Zhao \& Zhang (2000), Wu \& Dai (2005). Ganoderma sinense was long considered to be G. lucidum or G. japonicum by Chinese authors (Teng 1934, 1939, 1963). Ganoderma lucidum is characterized by cream or pinkish buff to clay buff colour basidiome, cuticle composed of clavate like cells and ellipsoid to broadly pear-shaped, coarsely echinulated basidiospores (Zhou et al. 2015). However, G. sinense differs from G. lucidum by having thin-fleshed basidiomes, with long, slender stipes, rarely branched skeletal hyphae with bovista type binding hyphae (Pegler \& Yao 1996). Ganoderma japonicum (= G. dimidiatum) has a yellow pileus when young, red-brown or rusty brown at maturity and off-white context (Pegler \& Yao 1996), but $G$. sinense has purplish black to black pileus and brown context. Ganoderma formosanum, introduced from Taiwan by Chang \& Chen (1984) has very similar morphology to $G$. sinense. However, Zhao \& Zhang (2000) considered G. formosanum as distinct based on its duplex context and ovoid basidiospores, and this conclusion was followed by Wu \& Dai (2005). Wang \& 
$\mathrm{Wu}$ (2007) studied both holotypes of G. formosanum and G. sinense and confirmed that both species share similar morphological characteristics. Furthermore, it was concluded that these species are synonyms based on molecular and morphological data and the earliest valid name to be used is G. sinense (Moncalvo et al. 1995a, Wang \& Wu 2007).
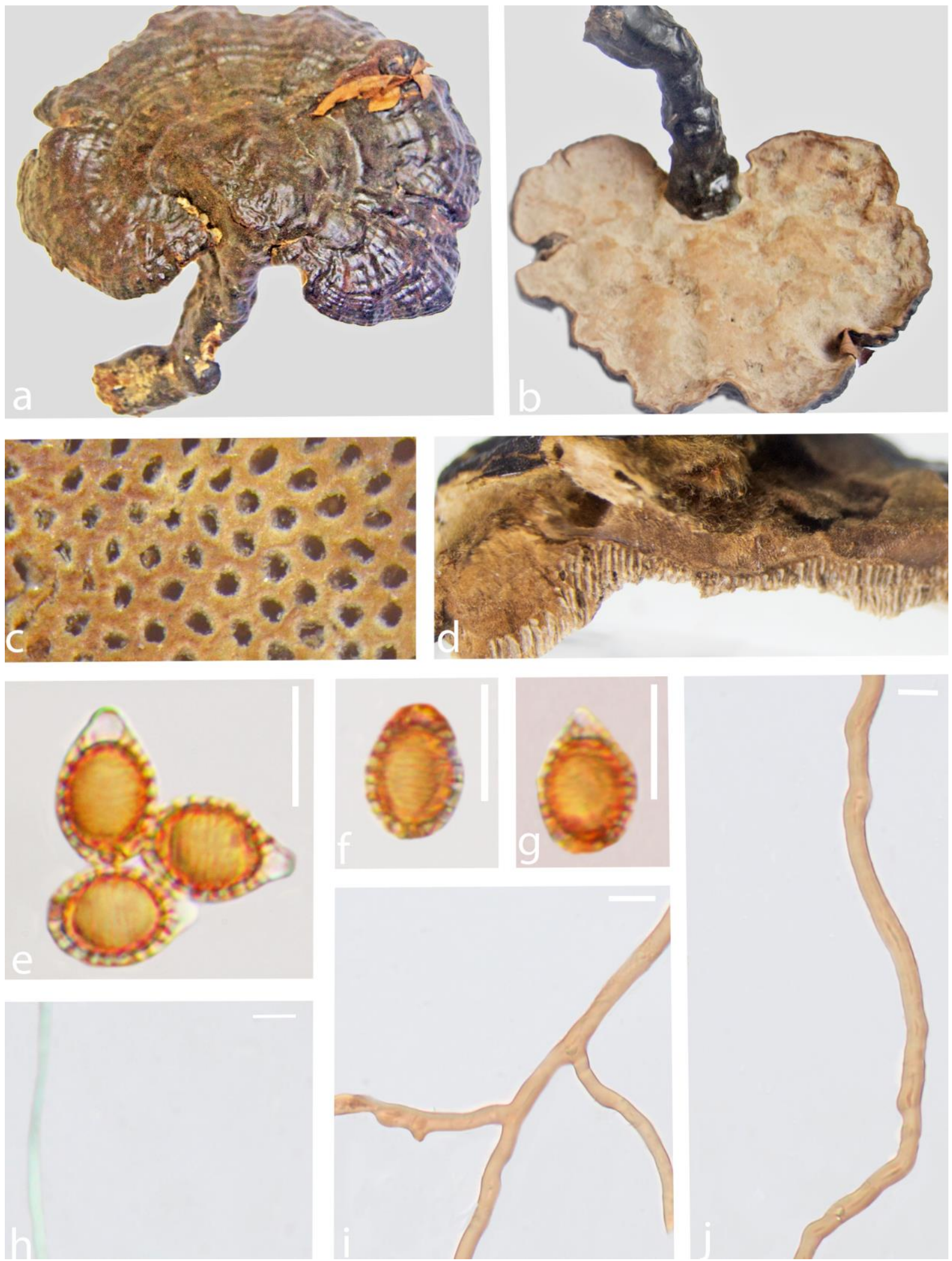

Fig. 18 - Ganoderma sinense specimen no. 17 (GACP170925130), a upper surface. b lower surface. c pores in the lower surface $(5 \times)$. d cut surface. e-g spores $(100 \times)$. h generative hyphae $(100 \times)$. i skeletal hyphae $(100 \times)$. j binding hyphae $(100 \times)$. Scale bars: $\mathrm{e}-\mathrm{g}=10 \mu \mathrm{m}, \mathrm{h}-\mathrm{j}=5 \mu \mathrm{m}$. 

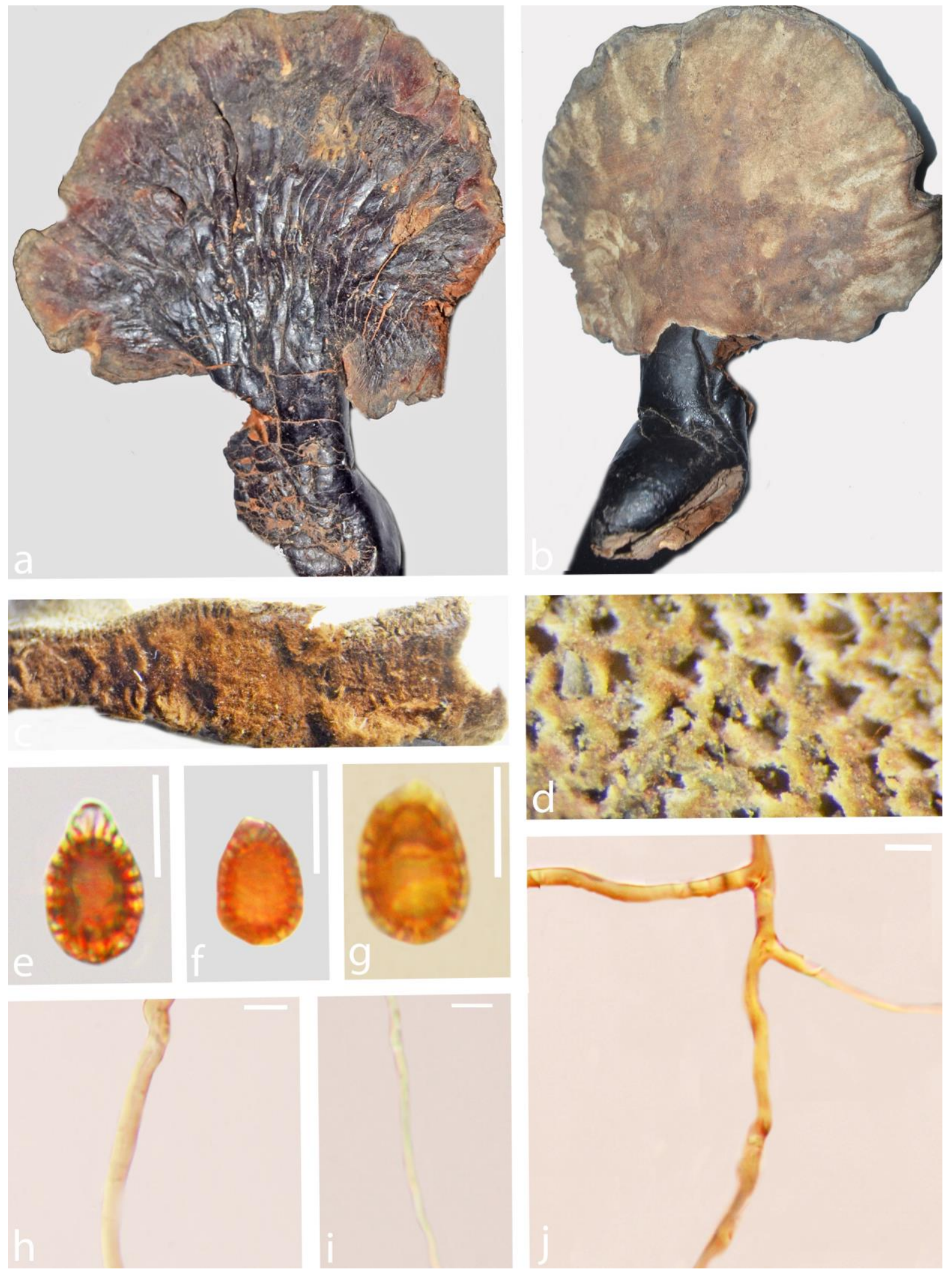

Fig. 19 - Ganoderma sinense specimen no. 18 (GACP17092543). a Upper surface. b Lower surface. c Cut surface. d Pores in the lower surface $(5 x)$. e-g Spores $(100 \times)$. h Skeletal hyphae (40x). i Generative hyphae (40x). j Binding hyphae (40x). Scale bars: $\mathrm{e}-\mathrm{g}=10 \mu \mathrm{m}, \mathrm{h}-\mathrm{j}=5 \mu \mathrm{m}$.

Ganoderma sinense is morphologically similar to G. orbiforme in having a purplish black to black laccate pileus, uniformly brown context or whitish streaks or patches near the cuticle, a dorsally lateral or lateral stipe and subtropical-tropical distribution (Wang et al. 2014). However, $G$. 
sinense can easily be distinguished from $G$. orbiforme since $G$. sinense bears an erect stipe, cuticle composed of clavate cells, and ovoid basidiospores with few, long and thick echinulae (Wang et al. 2014). Also, these 2 species have been clearly separated based on molecular data (Wang et al. 2014, Hapuarachchi et al. 2018b, this study).

In Pharmacopoeia of People's Republic of China 2000 edition (part one) the general term "Lingzhi" covers both "Chizhi (G. lucidum)" and "Zizhi" (G. sinense). Nevertheless, with the aid of molecular data, G. lucidum and G. sinense are clearly distinguished as two different taxa (Zhou et al. 2008, Liao et al. 2015, this study). Based on chemotaxonomical data, oxygenated lanostanetype triterpenes have been proven as suitable markers to differentiate G. lucidum and G. sinense (Fu et al. 2008).

\section{Discussion}

In this study, we retrieved all currently available ITS, LSU, SSU and RPB2 gene sequences of $G$. sinense and G. japonicum from GenBank. We observed, identical sequences of each gene separately; four G. sinense (Wei5327, Cui13835, ZZ, GACP14081236 ) and three G. japonicum (G22, Gja-1, AS5.69 type 1) ITS sequences obtained from GenBank together with 13 ITS sequences from our analysis (GACP17092520, GACP17092522, GACP17092530, GACP17092533, GACP17092543, GACP17092547, GACP17092581, GACP17092588, GACP17092592, GACP170925130, GACP17101242, GACP17101260, GACP17101270), one LSU sequence (Wei5327) accessed from GenBank and eight LSU sequences from this study (GACP17092533, GACP17092543, GACP17092567, GACP17092588, GACP17092512239, GACP17101254, GACP17101260, GACP17101270), eight SSU sequences from our analysis (GACP17092530， GACP17092533， GACP17092543， GACP17092567， GACP17092588, GACP17101254, GACP17101260, GACP17101270) and two RPB2 sequences, retrieved from GenBank (Wei5327 and Cui13835) and two sequences from our study (GACP17092543, GACP17092588), respectively. However, we noticed (reference sequence: G. sinense voucher Wei5327), seven G. sinense (GS92, GS96, GS111, GS175, GDGM25829, XZ-G-C1, XZ-G-C2) and one G. japonicum (AS5.69 type 2) ITS sequences retrieved from GenBank, together with six G. sinense ITS sequences from this study (GACP17092567, GACP17092588, GACP170925109, GACP170925130, GACP17101242, GACP17101254) show single-nucleotide polymorphism (SNP) located at position 158 in ITS 1 region (Fig. 20). However, they all grouped together to form a monophyletic clade in the phylogenetic tree (Fig. 1). Furthermore, there are no observed SNP's in $13 G$. sinense sequences from this study and three of each $G$. sinense and G. japonicum nucleotide sequences retrieved from GenBank. SNP leads to phenotypic plasticity of species of Ganoderma and as such could easily lead to specimens being misidentified as new taxa. This phenomenon has been previously observed in G. orbiforme (Wang et al. 2014) and G. lucidum (Zhang et al. 2017), and this is the first time to record SNP in G. sinense.

As previously mentioned, $G$. japonicum $(=G$. dimidiatum) and $G$. sinense are two different species based on morphological evidence. Nevertheless, all G. japonicum and G. sinense sequences clustered together in our analysis. Ganoderma japonicum was introduced as Boletus dimidiatus from Japan (Thunberg 1784) based on laterally stipitate basidiocarp with usually undulate, ferruginous and shiny upper surface, yellow margin and white pore surface. Fries (1821) synonymized this species with Polyporus lucidus (Curtis) Fr., and Fries (1838) assigned the epithet "japonicus" for $B$. dimidiatus and accepted it as a separate taxon. Both $P$. japonicus Fr. and $P$. lucidus var. japonicus Fr. names can be accepted for this species since Fries did not number this taxon in the same page (Papp 2016). Bresadola (1912) combined P. japonicus in Ganoderma and introduced it as a variety of G. lucidum. Sawada (1931) suggested that P. japonicus is a separate species and referred to it as G. japonicum (Fr.) Sawada by observing the darker form of G. lucidum. Imazeki (1939) suggested that $P$. japonicus sensu Fries is a synonym of Ganoderma lucidum. The original descriptions and drawings of both Boletus dimidiatus and P. japonicus, suggest that they represent a member of the G. lucidum species complex (Dai et al. 2017). However, G. lucidum sensu stricto is distributed in northern and southern Europe, and probably extends to China 
(Moncalvo et al. 1995a, b). Further studies confirmed that the species named G. lucidum from both Europe and mainland China are not conspecific based on analyses of ITS and 25S ribosomal DNA sequences. Later, many other authors confirmed the same opinion (Pegler \& Yao 1996, Smith \& Sivasithamparam 2000, Hong \& Jung 2004). The specimen examined by Fries has probably been lost (Moncalvo \& Ryvarden (1997) and P. japonicus Fr. is based on the type of Boletus dimidiatus, which was cited by Fries in 1838 (Papp 2016). Since the names published in Fries (1838) were not sanctioned, the binomial $P$. japonicus is illegitimate and is a superfluous name for $B$. dimidiatus. Hence, the new combination, Ganoderma dimidiatum (Thunb.) V. Papp was proposed as the earliest valid name to use for this species (Papp 2016).

\begin{tabular}{|c|c|c|}
\hline & & 170 \\
\hline$D$ Translate $D$ Consensus & & cecct \\
\hline G_sinense_Wei5327 (1>793) & $\rightarrow$ & TACACCIGIGCACITACIGIGGGITACGGGCIGIGGAGCGGGCICIGCGGAG \\
\hline G_sinense_Cuil38: & $\rightarrow$ & TACACCTGIGCACTIACTGIGGGITACGGGCTGIGGAGCGGGCTCIGCGGAG \\
\hline G_sinense_GACP171012 & & TACACCIGIGCACTIACIGIGGGITACGGGCIGIGGAGCGGGCTCIGCGGAGI \\
\hline G_sinense_GACP170925431 & $\rightarrow$ & TACACCTGTGCACTTACTGTGGGITACGGGCIGIGGAGCGGGCTCTGCGGA \\
\hline GS961 & $\rightarrow$ & TACACCTGIGCACTTACTGIGGGTIACGGr $\subset$ TGTGGAGCGGGCTCTGCGGAGC \\
\hline GS11 & $\rightarrow$ & TACACCIGIGCACTIACTGIGGGIIACGGrCTGIGGAGCGGGCICIGCGGAG \\
\hline G_si & $\rightarrow$ & TACACCTGTGCACTIACTGTGGGTTACGGrCIGTGGAGCGGGCTCTGCGGAd \\
\hline G_ja & & TACACCTGTGCACTIACTGIGGGITACGGGCTGIGGAGCGGGCTCTGCGGAG \\
\hline G_si & $\rightarrow$ & IACACCTGIGCACTTACTGTGGGITACGGGCTGIGGAGCGGGCTCTGCGGAG \\
\hline G_si & $\rightarrow$ & TACACCIGIGCACITACIGTGGGITACGGACIGIGGAGCGGGCICIGCGGAC \\
\hline$(1>793)$ & $\rightarrow$ & TACACCIGTGCACTTACTGTGGGTTACGGACTGIGGAGCGGGCTCTGCGGAG \\
\hline G_s: & $\rightarrow$ & TACACCTGIGCACTIACTGIGGGIIACGGGCTGIGGAGCGGGCTCIGCGGAG \\
\hline$G \_j a$ & $\rightarrow$ & TACACCIGTGCACTIACIGTGGGTTACGGGCTGIGGAGCGGGCTCTGCGGAG \\
\hline G_si & $\rightarrow$ & TACACCIGTGCACTIACTGTGGGTTACGGACIGIGGAGCGGGCTCTGCGGAC \\
\hline G_si & $\rightarrow$ & TACACCTGIGCACTTACIGTGGGTTACGGACTGIGGAGCGGGCTCTGCGGAG \\
\hline$G_{-j a}$ & & CGGGCIGIGGAGCGGGCTCTGCGGAG \\
\hline G_s: & $\rightarrow$ & CGGGCTGIGGAGCGGGCTCTGCGGAG \\
\hline G_s. & - & TACACCTGIGCACTIACTGIGGGITACGGGCTGIGGAGCGGGCTCIGCGGAG \\
\hline G_si & $\rightarrow$ & TACACCIGIGCACTIACTGIGGGTTACGGACIGIGGAGCGGGCICIGCGGAG \\
\hline G_si: & $\rightarrow$ & TACACCIGTGCACITACTGIGGGTTACGGACTGIGGAGCGGGCTCIGCGGAG \\
\hline G_sil & - & CGGGCTGIGGAGCGGGCTCTGCGGAG \\
\hline $\mathrm{G}_{-}^{-} \mathrm{s}$ & $\rightarrow$ & CGGGCTGTGGAGCGGGCTCTGCGGAG: \\
\hline G_s: & $\rightarrow$ & TACACCIGIGCACITACIGIGGGITACGGACIGIGGAGCGGGCICIGCGGAG \\
\hline G_si: & $\rightarrow$ & TACACCIGTGCACTIACIGTGGGITACGGACTGIGGAGCGGGCICTGCGGAG \\
\hline G_sil & $\rightarrow$ & TACACCTGTGCACTTACTGTGGGITACGGGCTGIGGAGCGGGCTCTGCGGAG \\
\hline G_si & - & TACACCTGTGCACTTACIGIGGGTTACGGGCTGIGGAGCGGGCTCTGCGGAG \\
\hline G_s: & - & TACACCIGIGCACTIACTGIGGGITACGGGCTGIGGAGCGGGCICIGCGGAG \\
\hline G_si & $\rightarrow$ & TACACCTGIGCACTIACTGIGGGITACGGGCTGIGGAGCGGGCTCTGCGGAG \\
\hline $1>793)$ & $\rightarrow$ & TACACCTGIGCACTIACTGTGGGTIACGGGCTGIGGAGCGGGCTCTGCGGAG \\
\hline XZ_G_C2 $(1>793)$ & $\rightarrow$ & TACACCTGTGCACTTACIGTGGGITACGGACTGTGGAGCGGGCTCTGCGGAG \\
\hline $\mathrm{XZ}_{-} \mathrm{G}_{-} \mathrm{Cl}(1>7$ & & TACACCIGIGCACTIACIGIGGGITACGGACTGIGGAGCGGGCTCTGCGGAG \\
\hline um_ $\bar{A} S \bar{S}$ _ 69 _t & & TACACCIGIGCACTIACIGIGGGITACGGACIGIGGAGCGGGCTCIGCGGI \\
\hline
\end{tabular}

Fig. 20 - Multiple-alignment of Ganoderma sinense and G. japonicum ITS1 sequences. A singlenucleotide polymorphism located at positions 158 is indicated in blue (Reference sequence: $G$. sinense voucher Wei5327). R stands for 'AG'.

Since, G. japonicum is a blackish, laccate, and stipitate fungus, it was generally synonymized with the traditional Chinese medicinal mushroom G. sinense distributed in subtropical areas (Teng 1964, Zhao 1989). However, it is most likely a misapplication, since specimens of G. japonicum from China and Japan are not conspecific as G. japonicum has a yellow pileus when young, red brown or rusty brown at maturity and off-white context, therefore is clearly distinguished from the purplish black to black pileus and brown context in G. sinense (Pegler \& Yao 1996, Dai et al. 2017). Moreover, in our collections we observed a brown context in every specimen, which in turn concluded them as $G$. sinense. Liao et al. (2015) suggested that $G$. sinense and G. japonicum should be considered as synonyms based on their high sequence similarity in ITS data. We also observed 
this scenario in our analysis, not only ITS (except SNP showing sequences), but with LSU, SSU and RBP2 sequence data. Despite that, all the available sequences of G. japonicum (Wang \& Yao 2005) in GenBank were obtained from Chinese specimens (descriptions unavailable) and were most likely wrongly identified. Also, still there are no molecular data available under the new authentic name "G. dimidiatum" in GenBank. Subsequently, we could not assure the authenticity of the distribution of $G$. dimidiatum in China since lack of molecular evidence from the type locality (Japan). Hence, based on molecular data, we conclude that the above studied materials from China are conspecific and represent one taxon; $G$. sinense. We would recommend recollecting fresh collections and reference collections from the type locality to clarify the taxonomy of $G$. dimidiatum. In addition, to obtain a better understanding of the evolution of $G$. dimidiatum and $G$. sinense, a phylogeny with more genes, and in particular single-copy nuclear genes such as TEF1 or $\beta$-tubulin would be recommended. Furthermore, a combination of morphological, chemotaxonomic and molecular methods would be more appropriate to develop a more stable taxonomy $G$. dimidiatum and $G$. sinense.

Our study reveals that the collection of $20 \mathrm{G}$. sinense specimens exhibit great variability in macro-morphology, yet the micro-morphological characteristics (hyphal system, basidiospore size and shape) do not show significant variation except one specimen; dimitic hyphal system (GACP17101254), pores circular, subcircular or isodiametric in shape and basidiospore size varies from 10-14 × 7-9 $\mu \mathrm{m}$ with ellipsoid shape (Table 2).

Among our $20 \mathrm{G}$. sinense specimens, 14 were from Sandu Shui County and five from Rongjiang County, Kaili, Guizhou Province, China. One specimen was from its type locality, Hainan Province, China (Table 3); this island has subtropical to tropical vegetation (Dai et al. 2011). Sandu Shui and Rongjiang Countries have a subtropical monsoon climate and subtropical humid monsoon climate, respectively (National Meteorological Information Center, China, 2000). As previously described, environmental factors such as temperature, humidity and air pressure vary according to the elevation of mountains (Table 3). Researchers have shown that the range and duration of light exposure play important roles in the formation of basidiomes in fungi such as Coprinus sp., Typhula ishikariensis and Clavicorona pyxidata (Morimoto \& Oda 1973, James \& McLaughlin 1988, Boulianne et al. 2000, Kawakami et al. 2004). The high frequency of G. sinense specimens found in southwestern Guizhou Province (Sandu and Kaili) in the rainy summer suggests that temperature and humidity are important factors in the formation and maturation of $G$. sinense. However, the temperature effect is not likely to be direct, as specimens were most commonly found under closed canopies where they reach greater length (GACP17092533, GACP17092592). Few researchers described the relationship between the extent of canopy coverage and light incidence in tropical zone mushrooms (Lodge \& Cantrell 1995, Suárez-Duque 2004, Gibertoni et al. 2007, Lodge et al. 2008). Steep slopes enhance basidiome development of Thelephora sp. due to factors related to humidity and nutrient requirements (Lodge et al. 2008). In this study, $G$. sinense specimens were found predominantly on steeper slopes due to improved nutrient uptake and with lower humidity. Hence, environmental factors such as air pressure, elevation, humidity, nutrient uptake, $\mathrm{pH}$ and temperature significantly vary in Hainan, Kaili and Sandu mountain areas which in turn affect basidiome development in G. sinense. In addition, slope, orientation and vegetation types in the collected area greatly affect the phenotypic plasticity (Ramírez-López et al. 2013).

Based on a combined analysis of the molecular results, related micro morphological data and environmental factors, we conclude that all 20 specimens examined in this study are conspecific even though the macro-morphology shows great variability. We suggest establishing Ganoderma sinense as a species complex based on molecular data. However, we need epitypes, reference collections and fresh collections with multigene nucleotide sequence data (with TEF1 or $\beta$-tubulin) to determine the taxonomy and evolution of $G$. sinense. A combination of morphological, chemotaxonomic, molecular and extrinsic factor determination methods would be suitable to establish a more stable taxonomy for $G$. sinense. Maximum taxon sampling, roles of conductivity 
and distribution of nutrients, structure and type of soils data will be needed to better understanding the development and distribution of this species.

Table 2 Morphological characteristics comparison for 20 Ganoderma sinense specimens observed in this study

\begin{tabular}{|c|c|c|c|c|c|c|c|c|c|c|c|c|}
\hline \multirow{2}{*}{$\begin{array}{l}\text { Specimen } \\
\text { voucher }\end{array}$} & \multicolumn{8}{|c|}{ Basidiome upper surface } & \multirow[t]{2}{*}{ Context } & \multirow{2}{*}{$\begin{array}{l}\text { Hyphal } \\
\text { system }\end{array}$} & \multicolumn{2}{|c|}{ Basidiospore } \\
\hline & $\begin{array}{l}\text { Stipe } \\
\text { orientation }\end{array}$ & Shape & $\begin{array}{l}\text { Size } \\
(\mathrm{cm})\end{array}$ & Colour & $\begin{array}{l}\text { Margin } \\
\text { colour }\end{array}$ & $\begin{array}{l}\text { Laccate/non } \\
\text { laccate }\end{array}$ & $\begin{array}{l}\text { Concentric } \\
\text { zones }\end{array}$ & $\begin{array}{l}\text { Radially } \\
\text { rugose }\end{array}$ & & & Shape & $\begin{array}{l}\text { Average } \\
\text { size } \\
(\mu \mathrm{m})\end{array}$ \\
\hline GACP17092567 & $\begin{array}{l}\text { Centrally } \\
\text { stipitate }\end{array}$ & Orbicular & $\begin{array}{l}7- \\
8.5 \times \\
6-8\end{array}$ & $\begin{array}{l}\text { Yellowish } \\
\text { brown to } \\
\text { pale } \\
\text { brown }\end{array}$ & Pale brown & $\begin{array}{l}\text { Strongly } \\
\text { laccate }\end{array}$ & $\begin{array}{l}\text { Weakly } \\
\text { concentrically } \\
\text { sulcate }\end{array}$ & $\begin{array}{l}\text { Not } \\
\text { observed }\end{array}$ & Duplex & Trimitic & Ellipsoid & $\begin{array}{l}11.5 \times \\
7.9\end{array}$ \\
\hline GACP17092533 & $\begin{array}{l}\text { Eccentrically } \\
\text { stipitate }\end{array}$ & Reniform & $\begin{array}{l}12- \\
15.5 \\
\times \\
10- \\
12.5\end{array}$ & $\begin{array}{l}\text { Yellowish } \\
\text { brown to } \\
\text { pale } \\
\text { brown } \\
\text { when } \\
\text { young, } \\
\text { becoming } \\
\text { reddish } \\
\text { brown to } \\
\text { brownish } \\
\text { orange } \\
\text { when } \\
\text { mature }\end{array}$ & $\begin{array}{l}\text { Concolorous } \\
\text { with the } \\
\text { pileus }\end{array}$ & $\begin{array}{l}\text { Strongly } \\
\text { laccate }\end{array}$ & $\begin{array}{l}\text { Distinctly } \\
\text { concentrically } \\
\text { sulcate }\end{array}$ & $\begin{array}{l}\text { Not } \\
\text { observed }\end{array}$ & Duplex & Trimitic & Ellipsoid & $\begin{array}{l}12.5 \times \\
8.3\end{array}$ \\
\hline GACP17092522 & $\begin{array}{l}\text { Eccentrically } \\
\text { stipitate }\end{array}$ & Suborbicular & $\begin{array}{l}5.5- \\
8 \times \\
3- \\
4.5\end{array}$ & $\begin{array}{l}\text { Yellowish } \\
\text { brown to } \\
\text { pale } \\
\text { brown }\end{array}$ & $\begin{array}{l}\text { Yellow } \\
\text { brown }\end{array}$ & Laccate & Not observed & $\begin{array}{l}\text { Slightly } \\
\text { radially } \\
\text { rugose }\end{array}$ & Duplex & Trimitic & Ellipsod & $\begin{array}{l}10.2 \times \\
7.2\end{array}$ \\
\hline GACP17092530 & $\begin{array}{l}\text { Centrally } \\
\text { stipitate }\end{array}$ & Orbicular & $\begin{array}{l}6-10 \\
\times 5- \\
8\end{array}$ & $\begin{array}{l}\text { Yellowish } \\
\text { brown to } \\
\text { pale } \\
\text { brown } \\
\text { when } \\
\text { young, } \\
\text { becoming } \\
\text { reddish }\end{array}$ & $\begin{array}{l}\text { Yellow } \\
\text { brown }\end{array}$ & $\begin{array}{l}\text { Strongly } \\
\text { laccate }\end{array}$ & $\begin{array}{l}\text { Weakly } \\
\text { concentrically } \\
\text { sulcate }\end{array}$ & $\begin{array}{l}\text { Not } \\
\text { observed }\end{array}$ & Duplex & Trimitic & $\begin{array}{l}\text { Ellipso- } \\
\text { id }\end{array}$ & $\begin{array}{l}11.6 \times \\
8.6\end{array}$ \\
\hline
\end{tabular}


Table 2 Continued.

\begin{tabular}{|c|c|c|c|c|c|c|c|c|c|c|c|c|}
\hline \multirow{2}{*}{$\begin{array}{l}\text { Specimen } \\
\text { voucher }\end{array}$} & \multicolumn{8}{|c|}{ Basidiome upper surface } & \multirow[t]{2}{*}{ Context } & \multirow{2}{*}{$\begin{array}{l}\text { Hyphal } \\
\text { system }\end{array}$} & \multicolumn{2}{|c|}{ Basidiospore } \\
\hline & $\begin{array}{l}\text { Stipe } \\
\text { orientation }\end{array}$ & Shape & $\begin{array}{l}\text { Size } \\
(\mathrm{cm})\end{array}$ & Colour & $\begin{array}{l}\text { Margin } \\
\text { colour }\end{array}$ & $\begin{array}{l}\text { Laccate/non } \\
\text { laccate }\end{array}$ & $\begin{array}{l}\text { Concentric } \\
\text { zones }\end{array}$ & $\begin{array}{l}\text { Radially } \\
\text { rugose }\end{array}$ & & & Shape & $\begin{array}{l}\text { Average } \\
\text { size } \\
(\mu \mathrm{m})\end{array}$ \\
\hline & & & & $\begin{array}{l}\text { brown to } \\
\text { brownish } \\
\text { orange } \\
\text { when } \\
\text { mature }\end{array}$ & & & & & & & & \\
\hline GACP170925128 & $\begin{array}{l}\text { Eccentrically } \\
\text { stipitate }\end{array}$ & Spathulate & $\begin{array}{l}7-9 \\
\times 7- \\
8\end{array}$ & $\begin{array}{l}\text { Yellowish } \\
\text { brown to } \\
\text { pale } \\
\text { brown } \\
\text { when } \\
\text { young, } \\
\text { becoming } \\
\text { reddish } \\
\text { brown to } \\
\text { brownish } \\
\text { orange } \\
\text { when } \\
\text { mature }\end{array}$ & $\begin{array}{l}\text { Concolorous } \\
\text { with the } \\
\text { pileus }\end{array}$ & $\begin{array}{l}\text { Strongly } \\
\text { laccate }\end{array}$ & Not observed & $\begin{array}{l}\text { Not } \\
\text { observed }\end{array}$ & Duplex & Trimitic & $\begin{array}{l}\text { Ellipso- } \\
\text { id }\end{array}$ & $\begin{array}{l}12.3 \times \\
8.6\end{array}$ \\
\hline GACP17092592 & $\begin{array}{l}\text { Eccentrically } \\
\text { stipitate, } \\
\text { having } \\
\text { dichotomous, } \\
\text { trichotomous } \\
\text { or irregular } \\
\text { branches }\end{array}$ & Subreniform & $\begin{array}{l}6- \\
10 \times \\
5-8\end{array}$ & $\begin{array}{l}\text { Grey to } \\
\text { brownish } \\
\text { grey }\end{array}$ & $\begin{array}{l}\text { Concolorous } \\
\text { with the } \\
\text { pileus }\end{array}$ & $\begin{array}{l}\text { Strongly } \\
\text { laccate }\end{array}$ & $\begin{array}{l}\text { Distinctly } \\
\text { concentrically } \\
\text { sulcate }\end{array}$ & $\begin{array}{l}\text { Slightly } \\
\text { radially } \\
\text { rugose }\end{array}$ & Duplex & Trimitic & $\begin{array}{l}\text { Broadly } \\
\text { ellipsoid }\end{array}$ & $\begin{array}{l}11.2 \times \\
8.1\end{array}$ \\
\hline GACP171012239 & $\begin{array}{l}\text { Eccentrically } \\
\text { stipitate }\end{array}$ & Reniform & $\begin{array}{l}6-8 \\
\times \\
3.5- \\
5.5\end{array}$ & $\begin{array}{l}\text { Brownish } \\
\text { grey to } \\
\text { black }\end{array}$ & $\begin{array}{l}\text { Concolorous } \\
\text { with the } \\
\text { pileus }\end{array}$ & Laccate & $\begin{array}{l}\text { Distinctly } \\
\text { concentrically } \\
\text { sulcate }\end{array}$ & Observed & Duplex & Trimitic & $\begin{array}{l}\text { Ellipso- } \\
\text { id }\end{array}$ & $\begin{array}{l}11.0 \times \\
7.5\end{array}$ \\
\hline GACP17092588 & $\begin{array}{l}\text { Eccentrically } \\
\text { stipitate }\end{array}$ & Subreniform & $\begin{array}{l}7- \\
10 \times \\
6-\end{array}$ & $\begin{array}{l}\text { Reddish } \\
\text { brown to } \\
\text { brownish }\end{array}$ & $\begin{array}{l}\text { Grayish } \\
\text { yellow }\end{array}$ & $\begin{array}{l}\text { Strongly } \\
\text { laccate }\end{array}$ & $\begin{array}{l}\text { Slightly } \\
\text { concentrically } \\
\text { sulcate }\end{array}$ & Observed & Triplex & Trimitic & $\begin{array}{l}\text { Ellipso- } \\
\text { id }\end{array}$ & $\begin{array}{l}12.2 \times \\
8.5\end{array}$ \\
\hline
\end{tabular}


Table 2 Continued.

\begin{tabular}{|c|c|c|c|c|c|c|c|c|c|c|c|c|}
\hline \multirow{2}{*}{$\begin{array}{l}\text { Specimen } \\
\text { voucher }\end{array}$} & \multicolumn{8}{|c|}{ Basidiome upper surface } & \multirow[t]{2}{*}{ Context } & \multirow{2}{*}{$\begin{array}{l}\text { Hyphal } \\
\text { system }\end{array}$} & \multicolumn{2}{|c|}{ Basidiospore } \\
\hline & $\begin{array}{l}\text { Stipe } \\
\text { orientation }\end{array}$ & Shape & $\begin{array}{l}\text { Size } \\
(\mathrm{cm})\end{array}$ & Colour & $\begin{array}{l}\text { Margin } \\
\text { colour }\end{array}$ & $\begin{array}{l}\text { Laccate/non } \\
\text { laccate }\end{array}$ & $\begin{array}{l}\text { Concentric } \\
\text { zones }\end{array}$ & $\begin{array}{l}\text { Radially } \\
\text { rugose }\end{array}$ & & & Shape & $\begin{array}{l}\text { Average } \\
\text { size } \\
(\mu \mathrm{m})\end{array}$ \\
\hline GACP14081236 & $\begin{array}{l}\text { Eccentrically } \\
\text { stipitate }\end{array}$ & Reniform & $\begin{array}{l}6.5 \\
7-8 \\
\times \\
5.0- \\
5.5\end{array}$ & $\begin{array}{l}\text { orange } \\
\text { Brownish } \\
\text { grey to } \\
\text { black }\end{array}$ & $\begin{array}{l}\text { Concolorous } \\
\text { with the } \\
\text { pileus }\end{array}$ & $\begin{array}{l}\text { Strongly } \\
\text { laccate }\end{array}$ & $\begin{array}{l}\text { Distinctly } \\
\text { concentrically } \\
\text { sulcate }\end{array}$ & $\begin{array}{l}\text { Slightly } \\
\text { radially } \\
\text { rugose }\end{array}$ & Duplex & Dimitic & Ellipsoid & $\begin{array}{l}12.3 \times \\
8.5\end{array}$ \\
\hline GACP17092543 & $\begin{array}{l}\text { Eccentrically } \\
\text { stipitate }\end{array}$ & Subreniform & $\begin{array}{l}7-10 \\
\times 6- \\
8\end{array}$ & $\begin{array}{l}\text { Brown to } \\
\text { brownish } \\
\text { black }\end{array}$ & $\begin{array}{l}\text { Yellow } \\
\text { brown }\end{array}$ & $\begin{array}{l}\text { Strongly } \\
\text { laccate }\end{array}$ & $\begin{array}{l}\text { Slightly } \\
\text { concentrically } \\
\text { sulcate }\end{array}$ & Observed & Duplex & Trimitic & Ellipsoid & $\begin{array}{l}11.4 \times \\
7.8\end{array}$ \\
\hline GACP17092548 & $\begin{array}{l}\text { Eccentrically } \\
\text { stipitate }\end{array}$ & Suborbicular & $\begin{array}{l}6-16 \\
\times 9- \\
11\end{array}$ & $\begin{array}{l}\text { Dark } \\
\text { brown to } \\
\text { brownish } \\
\text { black }\end{array}$ & $\begin{array}{l}\text { Yellow } \\
\text { brown }\end{array}$ & $\begin{array}{l}\text { Strongly } \\
\text { laccate }\end{array}$ & $\begin{array}{l}\text { Slightly } \\
\text { concentrically } \\
\text { sulcate }\end{array}$ & $\begin{array}{l}\text { Slightly } \\
\text { radially } \\
\text { rugose }\end{array}$ & Duplex & Trimitic & $\begin{array}{l}\text { Ellipso- } \\
\text { id }\end{array}$ & $\begin{array}{l}12.2 \times \\
8.2\end{array}$ \\
\hline GACP17101242 & $\begin{array}{l}\text { Eccentrically } \\
\text { stipitate }\end{array}$ & Subreniform & $\begin{array}{l}2.5- \\
4 \times \\
1.5- \\
2.5\end{array}$ & $\begin{array}{l}\text { Brownish } \\
\text { black }\end{array}$ & $\begin{array}{l}\text { Concolorous } \\
\text { with the } \\
\text { pileus }\end{array}$ & Laccate & $\begin{array}{l}\text { Slightly } \\
\text { concentrically } \\
\text { sulcate }\end{array}$ & Observed & Duplex & Trimitic & $\begin{array}{l}\text { Ellipso- } \\
\text { id }\end{array}$ & $\begin{array}{l}13.8 \times \\
9.1\end{array}$ \\
\hline GACP17101254 & $\begin{array}{l}\text { Eccentrically } \\
\text { stipitate }\end{array}$ & Subreniform & $\begin{array}{l}1.5- \\
4.5 \times \\
1.0- \\
2.5\end{array}$ & $\begin{array}{l}\text { Brownish } \\
\text { black }\end{array}$ & $\begin{array}{l}\text { Concolorous } \\
\text { with the } \\
\text { pileus }\end{array}$ & Laccate & $\begin{array}{l}\text { Distinctly } \\
\text { concentrically } \\
\text { sulcate }\end{array}$ & Observed & Duplex & Dimitic & $\begin{array}{l}\text { Ellipso- } \\
\text { id }\end{array}$ & $\begin{array}{l}12.5 \times \\
8.5\end{array}$ \\
\hline GACP17101260 & $\begin{array}{l}\text { Eccentrically } \\
\text { stipitate }\end{array}$ & Reniform & $\begin{array}{l}2.5- \\
5 \times \\
1.0- \\
2.5\end{array}$ & $\begin{array}{l}\text { Light } \\
\text { brown }\end{array}$ & $\begin{array}{l}\text { Concolorous } \\
\text { with the } \\
\text { pileus }\end{array}$ & Laccate & $\begin{array}{l}\text { Slightly } \\
\text { concentric, } \\
\text { not sulcate }\end{array}$ & Observed & Triplex & Trimitic & $\begin{array}{l}\text { Ellipso- } \\
\text { id }\end{array}$ & $\begin{array}{l}12.9 \times \\
8.9\end{array}$ \\
\hline GACP17101270 & $\begin{array}{l}\text { Eccentrically } \\
\text { stipitate }\end{array}$ & Flabelliform & $\begin{array}{l}2-4 \\
\times 1- \\
1.5\end{array}$ & $\begin{array}{l}\text { Reddish } \\
\text { brown to } \\
\text { brownish } \\
\text { black }\end{array}$ & $\begin{array}{l}\text { Yellowish } \\
\text { brown }\end{array}$ & Laccate & Not observed & $\begin{array}{l}\text { Slightly } \\
\text { radially } \\
\text { rugose }\end{array}$ & Duplex & Trimitic & $\begin{array}{l}\text { Ellipso- } \\
\text { id }\end{array}$ & $\begin{array}{l}12.8 \times \\
2.9\end{array}$ \\
\hline GACP17092581 & $\begin{array}{l}\text { Eccentrically } \\
\text { stipitate }\end{array}$ & Spathulate & $\begin{array}{l}8-12 \\
\times 7- \\
9\end{array}$ & $\begin{array}{l}\text { Dark } \\
\text { brown to } \\
\text { brownish } \\
\text { black }\end{array}$ & $\begin{array}{l}\text { Concolorous } \\
\text { with the } \\
\text { pileus }\end{array}$ & Laccate & Observed & $\begin{array}{l}\text { Slightly } \\
\text { radially } \\
\text { rugose }\end{array}$ & Duplex & Trimitic & $\begin{array}{l}\text { Ellipso- } \\
\text { id }\end{array}$ & $\begin{array}{l}11.3 \times \\
8.0\end{array}$ \\
\hline
\end{tabular}


Table 2 Continued.

\begin{tabular}{|c|c|c|c|c|c|c|c|c|c|c|c|c|}
\hline \multirow{2}{*}{$\begin{array}{l}\text { Specimen } \\
\text { voucher }\end{array}$} & \multicolumn{8}{|c|}{ Basidiome upper surface } & \multirow[t]{2}{*}{ Context } & \multirow{2}{*}{$\begin{array}{l}\text { Hyphal } \\
\text { system }\end{array}$} & \multicolumn{2}{|c|}{ Basidiospore } \\
\hline & $\begin{array}{l}\text { Stipe } \\
\text { orientation }\end{array}$ & Shape & $\begin{array}{l}\text { Size } \\
(\mathrm{cm})\end{array}$ & Colour & $\begin{array}{l}\text { Margin } \\
\text { colour }\end{array}$ & $\begin{array}{l}\text { Laccate/non } \\
\text { laccate }\end{array}$ & $\begin{array}{l}\text { Concentric } \\
\text { zones }\end{array}$ & $\begin{array}{l}\text { Radially } \\
\text { rugose }\end{array}$ & & & Shape & $\begin{array}{l}\text { Average } \\
\text { size } \\
(\mu \mathrm{m})\end{array}$ \\
\hline GACP17092520 & $\begin{array}{l}\text { Eccentrically } \\
\text { stipitate }\end{array}$ & Spathulate & $\begin{array}{l}4- \\
5.5 \times \\
3-4\end{array}$ & $\begin{array}{l}\text { Dark } \\
\text { brown to } \\
\text { brownish } \\
\text { black }\end{array}$ & $\begin{array}{l}\text { Concolorous } \\
\text { with the } \\
\text { pileus }\end{array}$ & $\begin{array}{l}\text { Strongly } \\
\text { laccate }\end{array}$ & Not observed & Observed & Duplex & Trimitic & $\begin{array}{l}\text { Ellipso- } \\
\text { id }\end{array}$ & $\begin{array}{l}11.3 \times \\
8.2\end{array}$ \\
\hline GACP17092513 & $\begin{array}{l}\text { Eccentrically } \\
\text { stipitate }\end{array}$ & Reniform & $\begin{array}{l}7.5- \\
11 \times \\
6- \\
7.5\end{array}$ & $\begin{array}{l}\text { Dark } \\
\text { brown to } \\
\text { brownish } \\
\text { black }\end{array}$ & $\begin{array}{l}\text { Concolorous } \\
\text { with the } \\
\text { pileus }\end{array}$ & Laccate & Observed & Observed & Duplex & Trimitic & $\begin{array}{l}\text { Ellipso- } \\
\text { id }\end{array}$ & $\begin{array}{l}10.2 \times \\
7.0\end{array}$ \\
\hline
\end{tabular}

Table 3 Collection site details of Ganoderma sinense specimens in China

\begin{tabular}{|c|c|c|c|c|c|c|c|c|c|c|c|}
\hline Province & $\begin{array}{l}\text { Collection } \\
\text { site }\end{array}$ & $\begin{array}{l}\text { Collection } \\
\text { date }\end{array}$ & $\begin{array}{l}\text { Collec- } \\
\text { tor }\end{array}$ & $\begin{array}{l}\text { Ganoderma } \\
\text { sinense } \\
\text { specimen } \\
\text { voucher }\end{array}$ & $\begin{array}{l}\text { Longitudes } \\
\text { and } \\
\text { latitudes }\end{array}$ & $\begin{array}{l}\text { Average } \\
\text { annual } \\
\text { rain fall } \\
(\mathrm{mm})\end{array}$ & $\begin{array}{l}\text { Average } \\
\text { annual } \\
\text { temperature } \\
\left({ }^{\circ} \mathrm{C}\right)\end{array}$ & $\begin{array}{l}\text { Height } \\
\text { (m) }\end{array}$ & $\begin{array}{l}\text { Relati- } \\
\text { ve } \\
\text { Humi- } \\
\text { dity }(\%)\end{array}$ & Substrate- & Reference \\
\hline Hainan & $\begin{array}{l}\text { Jiangfengling } \\
\text { Mountain, } \\
\text { Le Dong } \\
\text { County }\end{array}$ & $2014 / 08 / 12$ & T.C. Wen & GACP14081236 & $\begin{array}{l}108^{\circ} 51^{\prime}- \\
109^{\circ} 02^{\prime} \mathrm{E} \\
18^{\circ} 44^{\prime} 18^{\circ} \\
52^{\prime} \mathrm{N}\end{array}$ & 2650 & 29 & 838 & 57 & $\begin{array}{l}\text { On rotten } \\
\text { wood, in } \\
\text { dry } \\
\text { dipterocarp } \\
\text { forest and } \\
\text { in upper } \\
\text { mixed } \\
\text { deciduous } \\
\text { forest or } \\
\text { growing up } \\
\text { from soil }\end{array}$ & Dai et al. 2011 \\
\hline Guizhou & $\begin{array}{l}\text { Sandu Shui } \\
\text { Autonomous } \\
\text { County }\end{array}$ & $2017 / 09 / 25$ & T.C. Wen & $\begin{array}{l}\text { GACP17092520, } \\
\text { GACP17092521, } \\
\text { GACP17092522, } \\
\text { GACP17092523, }\end{array}$ & $\begin{array}{l}107^{\circ} 40^{\prime}- \\
108^{\circ} 14^{\prime} \mathrm{E}, \\
25^{\circ} 30^{\prime}-\end{array}$ & 1326 & 18 & 560 & 80 & & $\begin{array}{l}\text { National } \\
\text { Meteorological } \\
\text { Information } \\
\text { Center, }\end{array}$ \\
\hline
\end{tabular}


Table 3 Continued.

\begin{tabular}{|c|c|c|c|c|c|c|c|c|c|c|c|}
\hline Province & $\begin{array}{l}\text { Collection } \\
\text { site }\end{array}$ & $\begin{array}{l}\text { Collection } \\
\text { date }\end{array}$ & $\begin{array}{l}\text { Collec- } \\
\text { tor }\end{array}$ & $\begin{array}{l}\text { Ganoderma } \\
\text { sinense } \text { specimen } \\
\text { voucher }\end{array}$ & $\begin{array}{l}\text { Longitudes } \\
\text { and } \\
\text { latitudes }\end{array}$ & $\begin{array}{l}\text { Average } \\
\text { annual } \\
\text { rain fall } \\
(\mathrm{mm})\end{array}$ & $\begin{array}{l}\text { Average } \\
\text { annual } \\
\text { temperature } \\
\left({ }^{\circ} \mathrm{C}\right)\end{array}$ & $\begin{array}{l}\text { Height } \\
\text { (m) }\end{array}$ & $\begin{array}{l}\text { Relati- } \\
\text { ve } \\
\text { Humi- } \\
\text { dity }(\%)\end{array}$ & Substrate- & Reference \\
\hline & & & & $\begin{array}{l}\text { GACP17092533, } \\
\text { GACP17092534, } \\
\text { GACP17092530, } \\
\text { GACP17092532 } \\
\text { GACP17092539, } \\
\text { GACP17092540, } \\
\text { GACP17092592, } \\
\text { GACP17092595 } \\
\text { GACP17092567, } \\
\text { GACP17092568 } \\
\text { GACP17092581, } \\
\text { GACP17092582, } \\
\text { GACP17092588, } \\
\text { GACP17092589 } \\
\text { GACP17092543, } \\
\text { GACP17092547, } \\
\text { GACP17092548, } \\
\text { GACP170925109, } \\
\text { GACP17092520, } \\
\text { GACP170925128, } \\
\text { GACP170925129, } \\
\text { GACP170925130, } \\
\text { GACP170925132 } \\
\text { GACP17101242, } \\
\text { GACP17101245 } \\
\text { GACP17101254, } \\
\text { GACP17101255 } \\
\text { GACP17101260, } \\
\text { GACP17101262 }\end{array}$ & $26^{\circ} 10^{\prime} \mathrm{N}$ & & & $\begin{array}{l}570 \\
600 \\
580 \\
520 \\
620 \\
650 \\
560 \\
580 \\
600\end{array}$ & 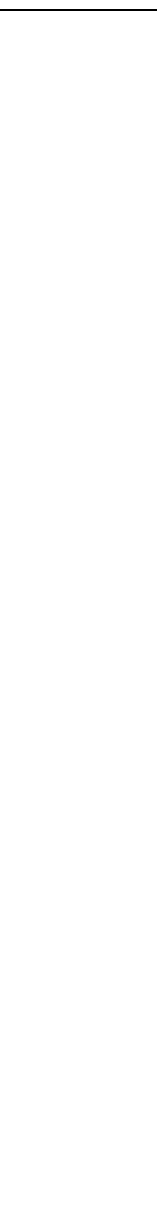 & & China, 2000 \\
\hline
\end{tabular}




\section{Acknowledgements}

This work was financed by the Science and Technology Foundation of Guizhou Province (No. [2017]2511-1), and the Science Research Foundation of Guizhou University (No. 201309). Samantha C. Karunarathna thanks CAS President's International Fellowship Initiative (PIFI) for funding his postdoctoral research (number 2018PC0006) and the National Science Foundation of China (NSFC) for funding this work under the project code 31750110478. Kalani K. Hapuarachchi is grateful to Dr. Olivier Raspé, Dr. Sajeewa Maharachchikumbura, Hansika Perera, Ishani Goonasekara and H.D. Yang for their valuable comments and suggestions.

\section{References}

Arulpandi I, Kalaichelvan PT. 2013 - Ganoderma adspersum and Ganoderma cupreum from South India, first report based on molecular phylogeny. International Journal of Current Microbiology and Applied Sciences (12), 693-702. doi: 10.5941/MYCO.2013.41.4.248

Aubin-Horth NA, Renn SC. 2009 - Genomic reaction norms: using integrative biology to understand molecular mechanisms of phenotypic plasticity. Molecular Ecology 18(18), 37633780. doi: 10.1111/j.1365-294X.2009.04313.x

Benson DA, Cavanaugh M, Clark K, Karsch-Mizrachi I et al. 2017 - GenBank. Nucleic Acids Research 45(D1): D37-D42. doi.org/10.1093/ nar/gkw1070

Bi ZS, Zheng G, Li TH. 1993 - The Macro fungus Flora of China's Guangdong Province, 119.

Boulianne RP, Liu Y, Aebi M, Lu BC et al. 2000 - Fruiting body development in Coprinus cinereus: regulated expression of two galectins secreted by a non-classical pathway. Microbiology 146, 1841-1853. doi:10.1099/00221287-146-8-1841

Bresadola G. 1912 - Fungi Congoenses. Annales Mycologici 9, 266-276.

Bujakiewicz A. 1992 - Macrofungi on soil in deciduous forest, p. 49-78. In: W. Winterhoff (ed.). Fungi in vegetation science. Kluwer Academic, Dordrecht, Holland.

Cao Y, Wu SH, Dai YC. 2012 - Species clarification of the prize medicinal Ganoderma mushroom “'Lingzhi'. Fungal Diversity 56, 49-62. doi.org/10.1007/s13225-012-0178-5

Chang TT, Chen T. 1984 - Ganoderma formosanum sp. nov. on Formosan sweet gum in Taiwan. Transactions of the British Mycological Society 82, 731-733. doi.org/10.1016/S0007-1536 (84)80119-9

Coetzee MP, Marincowitz S, Muthelo VG, Wingfield MJ. 2015 - Ganoderma species, including new taxa associated with root rot of the iconic Jacaranda mimosifolia in Pretoria, South Africa. IMA Fungus 6, 249-256. doi.org/10.5598/imafungus.2015.06.01.16

Costa-Rezende DH, Robledo GL, Goes-Neto A, Reck MA et al. 2017 - Morphological reassessment and molecular phylogenetic analyses of Amauroderma s.lat. raised new perspectives in the generic classification of the Ganodermataceae family. Persoonia 39, 254269. doi: 10.3767/persoonia.2017.39.10

Crous PW, Wingfield MJ, Le Roux JJ, Richardson DM et al. 2015 - Fungal Planet description sheets: 371-399. Persoonia: Molecular Phylogeny and Evolution of Fungi 35, 264-327. doi: 10.3767/003158515X690269.

Crous PW, Wingfield MJ, Richardson DM, Le Roux JJ et al. 2016 - Fungal Planet description sheets: 400-468. Persoonia: Molecular Phylogeny and Evolution of Fungi 36, 316-458. doi: 10.3767/003158516X692185.

Crous PW, Wingfield MJ, Burgess TI, Hardy GSJ et al. 2017 - Fungal Planet description sheets: 558-624. Persoonia: Molecular Phylogeny and Evolution of Fungi 38, 240-384. doi: $10.3767 / 003158517 X 698941$

Dai YC, Cui BK, Yuan HS, Li BD. 2007 - Pathogenic wood-decaying fungi in China. Forest Pathology 37, 105-120. doi.org/10.1111/j.1439-0329.2007.00485.x

Dai YC, Yang ZL, Cui BK, Yu CJ et al. 2009 - Species diversity and utilization of medicinal mushrooms and fungi in China. International Journal of Medicinal Mushrooms 11, 287-302. doi.org/10.1615/IntJMedMushr.v11.i3.80 
Dai YC, Cui BK, Yuan HS, He SH et al. 2011 - Wood-inhabiting fungi in southern China. 4. Polypores from Hainan Province. Annales Botanici Fennici 48, 219-231.

Dai YC, Zhou LW, Hattori T, Cao Y et al. 2017 - Ganoderma lingzhi (Polyporales, Basidiomycota): the scientific binomial for the widely cultivated medicinal fungus Lingzhi. Mycological Progress 16, 1051-1055. doi.org/10.1007/s11557-017-1347-4

De Silva DD, Rapior S, Fons F, Bahkali AH et al. 2012a - Medicinal mushrooms in supportive cancer therapies: an approach to anti-cancer effects and putative mechanisms of action. Fungal Diversity 55, 1-35. doi.org/10.1007/s13225-012-0151-3

De Silva DD, Rapior S, Hyde KD, Bahkali AH. 2012b - Medicinal mushrooms in prevention and control of diabetes mellitus. Fungal Diversity 56, 1-29. doi.org/10.1007/s13225-012-0187-4

De Silva DD, Rapior S, Sudarman E, Stadler M et al. 2013 - Bioactive metabolites from macrofungi: ethnopharmacology, biological activities and chemistry. Fungal Diversity 62, 140. doi.org/10.1007/s13225-013-0265-2

Fries EM. 1821 - Systema Mycologicum. 1, Lundae, 520.

Fries EM 1838 - Epicrisis Systematis Mycologici. Upsaliae, 610.

Fu CM, Lu GH, Schmitz OJ, Li ZW et al. 2008 - Improved chromatographic fingerprints for facile differentiation of two Ganoderma spp. Biomedical Chromatography 23(3), 280-288. doi.org/10.1002/bmc.1111.

Gibertoni TB, Santos PJP, Cavalcanti MAQ. 2007 - Ecological aspects of Aphyllophorales in the Atlantic rain forest in Northeast Brazil. Fungal Diversity 25, 49-67.

Gottlieb AM, Wright JE. 1999 - Taxonomy of Ganoderma from southern South America: subgenus Ganoderma. Mycological Research 103, 661-673. doi.org/10.1017/S0953756298007941

Hall TA. 1999 - BioEdit: a user-friendly biological sequence alignment editor and analysis program for Windows 95/98/NT. In: Nucleic Acids Symposium Series 41, 95-98.

Hapuarachchi KK, Wen TC, Deng CY, Kang JC et al. 2015 - Mycosphere Essays 1: Taxonomic confusion in the Ganoderma lucidum species complex. Mycosphere 6, 542-559. doi: 10.5943/mycosphere/6/5/4.

Hapuarachchi KK, Wen TC, Jeewon R, Wu XL et al. 2016a - Mycosphere Essays 7. Ganoderma lucidum-are the beneficial anti-cancer properties substantiated? Mycosphere 7(3), 305-332. doi: 10.5943/mycosphere/7/3/6.

Hapuarachchi KK, Wen TC, Jeewon R, Wu XL et al. 2016b - Mycosphere Essays 15. Ganoderma lucidum-are the beneficial medical properties substantiated? Mycosphere 7(3), 687-715. doi: 10.5943/mycosphere/7/6/1.

Hapuarachchi KK, Cheng CR, Wen TC, Jeewon R et al. 2017 - Mycosphere Essays 20: Therapeutic potential of Ganoderma species: insights into its use as traditional medicine. Mycosphere 8(10), 1653-1694. doi: 10.5943/mycosphere/8/10/5.

Hapuarachchi KK, Elkhateeb WA, Karunarathna SC, Cheng CR et al. 2018a - Current status of global Ganoderma cultivation, products, industry and market. Mycosphere 9(5), 1025-1052, doi 10.5943/mycosphere/9/5/6

Hapuarachchi KK, Karunarathna SC, Raspé O, De Silva KHWL et al. 2018b - High diversity of Ganoderma and Amauroderma (Ganodermataceae, Polyporales) in Hainan Island, China. Mycosphere 9(5), 931-982. doi 10.5943/mycosphere/9/5/1

Hapuarachchi KK, Karunarathna SC, Phengsintham P, Kakumyan P et al. 2018c - Amauroderma (Ganodermataceae, Polyporales) - bioactive compounds, beneficial properties and two new records from Laos. Asian Journal of Mycology 1, 121-136. doi.org/10.5943/ajom/1/1/10

Hillis DM, Bull JJ. 1993 - An empirical test of bootstrapping as a method for assessing confidence in phylogenetic analysis. Systematic Biology 42, 182-192. doi.org/10.1093/sysbio/42.2.182

Hong KK, Geon SS, Hong GK. 2001 - Comparison of characteristics of Ganoderma lucidum according to geographical origins: consideration of morphological characteristics. Micobiology 29, 80-84. doi.org/10.1080/12298093.2001.12015765 
Hong SG, Jeong W, Jung HS. 2002 - Amplification of mitochondrial small subunit ribosomal DNA of polypores and its potential for phylogenetic analysis. Mycologia 94, 823-833.

Hong SG, Jung HS. 2004 - Phylogenetic analysis of Ganoderma based on nearly complete mitochondrial small-subunit ribosomal DNA sequences. Mycologia 96, 742-755.

Imazeki R. 1939 - Studies on Ganoderma of Nippon. - Bulletin of the Natural Science Museum, Tokyo, 1, 29-52 (in Japanese).

Index Fungorum. 2019 - http://www.indexfungorum.org (accessed 27 February 2019).

James SW, McLaughlin DJ. 1988 - The influence of carbohydrate source and concentration and light on fruit body development in Clavicorona pyxidata. Mycologia 80, 89-98. doi org/:10.2307/3807498

Jeewon R, Hyde KD. 2016 - Establishing species boundaries and new taxa among fungi: recommendations to resolve taxonomic ambiguities. Mycosphere 7(11), 1669-1677. doi 10.5943/mycosphere/7/11/4

Jargalmaa S, Eimes JA, Park MS, Park JY et al. 2017 - Taxonomic evaluation of selected Ganoderma species and database sequence validation. Peer J, 5, p.e3596.

Karsten PA .1881 - Enumeralio boletinearum et polypore arum fennicarum, systemate novo dispositarum. Revue de Mycologie 3, 16-19.

Kawakami A, Matsumoto N, Naito S. 2004 - Environmental factors influencing sporocarp formation in Typhula ishikariensis. Journal of General Plant Pathology 70, 1-6. doi.org/10.1007/s10327-003-0086-3

Katoh K, Standley DM. 2013 - MAFFT multiple sequence alignment software version 7: improvements in performance and usability. Molecular biology and Evolution 30, 772-780. doi.org/10.1093/molbev/mst010.

Kornerup A, Wanscher JH. 1978 -Methuen Handbook of Color. Eyre Methuen, London,

Kost G. 1992 - Macrofungi on soil coniferous forest, p. 79-111. In: Winterhoff W (ed.). Fungi in Vegetation Science. Kluwer Academic, Dordrecht, Holland.

Li TH, Hu HP, Deng WQ, Wu SH et al. 2015 - Ganoderma leucocontextum, a new member of the G. lucidum complex from southwestern China. Mycoscience 56, 81-85. doi.org/10.1016/j.myc.2014.03.005

Liao B, Chen X, Han J, Dan Y et al. 2015 - Identification of commercial Ganoderma (Lingzhi) species by ITS2 sequences. Chinese Medicine 10(1), 22. doi: 10.1186/s13020-015-0056-7

Liu YJ, Whelen S, Hall BD. 1999 - Phylogenetic relationships among ascomycetes: evidence from an RNA polymerase II subunit. Molecular Biology and Evolution 16, 1799-1808. doi.org/10.1093/oxfordjournals.molbev.a026092

Lodge DJ, Cantrell S. 1995 - Fungal communities in wet tropical forests: variation in time and space. Canadian Journal of Botany 73, 1391-S1398.

Lodge DJ, McDowell WH, Macy J, Ward SK et al. 2008 - Distribution and role of mat-forming saprobic Basidiomycetes in a Tropical Forest 197-209. In: Boddy L, Frankland J.C, West PV (eds.). Ecology of Saprotrophic Basidiomycetes. Academic, London, England.

Moore-Landecker E. 1996 - Fundamentals of the Fungi. Prentice Hall, Upper Saddle River, New Jersey, USA.

Moore D, Gange AC, Gange EG, Boddy L. 2008 - Fruit bodies: their production and development in relation to environment, p. 79-103. In: Boddy L, Frankland J.C, West PV(eds.). Ecology of Saprotrophic Basidiomycetes. Academic, London, England.

Moore D, Robson GD, Trinci APJ. 2011 - 21st Century Guidebook to Fungi. Cambridge University, Cambridge, England.

Moncalvo JM, Wang HF, Hseu RS. 1995a - Gene phylogeny of the Ganoderma lucidum complex based on ribosomal DNA sequences. Comparison with traditional taxonomic characters. Mycological Research 99, 1489-1499. doi.org/10.1016/S0953-7562(09)80798-3

Moncalvo JM, Wang HH, Hseu RS. 1995b - Phylogenetic relationships in Ganoderma inferred from the internal transcribed spacers and 25S ribosomal DNA sequences. Mycologia 87(2), 223-238. doi.org/10.1080/00275514.1995.12026524 
Moncalvo JM, Ryvarden L. 1997 - A nomenclatural study of the Ganodermataceae Donk. Fungi flora $10,1-114$.

Moncalvo JM. 2005 - Molecular systematic in Ganoderma: what is Reishi? International Journal of Medicinal Mushrooms 7(3), 353-354. doi.org 10.1615/IntJMedMushrooms.v7.i3.160

Morimoto N, Oda Y. 1973 - Effects of light on fruit-body formation in a basidiomycete, Coprinus macrorhizus. Plant Cell and Physiology 14, 217-225. https://doi.org/10.1093/oxfordjournals.pcp.a074854

Miller RNG, Holderness M, Bridge PD, Chung GF et al. 1999 - Genetic diversity of Ganoderma in oil palm plantings. Plant Pathology 48, 595-603. doi.org/10.1046/j.1365-3059.1999.00390.x

Miller RE, Blair PD. 2009 - Input-output analysis: foundations and extensions. Cambridge University Press. doi.org/10.1017/CBO9780511626982

National Meteorological Information Center, China. 2000 - http://data.cma.cn

Nilsson RH, Tedersoo L, Abarenkov K. 2012 - Five simple guidelines for establishing basic authenticity and reliability of newly generated fungal ITS sequences. MycoKeys 4, 37-63. doi.org/10.3897/mycokeys.4.3606

Nylander JAA. 2004 - MrModeltest v2.2. Program distributed by the author: 2. Evolutionary Biology Centre, Uppsala University 1-2.

Papp V. 2016 - The first validly published laccate Ganoderma species from East Asia: $G$. dimidiatum comb. nov., the correct name for G. japonicum. Studia botanica hungarica 47(2), 263-268. doi org/10.17110/StudBot.2016.47.2.263

Pawlik A, Janusz G, Dębska I, Siwulski M et al. 2015 - Genetic and metabolic intraspecific biodiversity of Ganoderma lucidum. BioMed research international 2015, Article ID 726149, 13 pages. doi.org/10.1155/2015/726149

Pegler DN, Yao YJ. 1996 - Oriental species of Ganoderma section Ganoderma. In: Wasser SP (ed). Botany and mycology for the next millennium: collection of scientific articles devoted to the 70th Anniversary of Academician Sytnik KM. Kyiv: Kholodny NG Institute of Botany, National Academy of Sciences of Ukraine, 336-347.

Pilotti CA, Sanderson FR, Aitken EAB. 2003 - Genetic structure of a population of Ganoderma boninense on oil palm. Plant Pathology 52, 455-63. doi.org/10.1046/j.13653059.2003.00870.x

Pilotti CA, Sanderson FR, Aitken AB, Armstrong W. 2004 - Morphological variation and host range of two Ganoderma species from Papua New Guinea. Mycopathologia 158, 251-265.

Rambaut A. 2012 - FigTree version 1.4.0. (http://tree.bio.ed.ac.uk/software/figtree/).

Rambaut A, Suchard MA, Xie D, Drummond AJ. 2013 - Tracer version 1.6. University of Edinburgh. [Online]. (Accessed on 24.11.2018) available at http://tree.bio.ed.ac.uk/software/tracer

Ramírez-López, I, Villegas Ríos, M, Cano-Santana Z. 2013 - Phenotypic plasticity of the basidiomata of Thelephora sp. (Thelephoraceae) in tropical forest habitats. Revista de Biologica Tropical 61(1), 343-350.

Rannala B, Yang Z. 1996 - Probability distribution of molecular evolutionary trees: a new method of phylogenetic inference. Journal of Molecular Evolution 43(3), 304-311. doi.org/10.1007/BF02338839

Rehner SA, Samuels GJ. 1994 - Taxonomy and phylogeny of Gliocladium analysed from nuclear large subunit ribosomal DNA sequences. Mycological Research 98, 625-634. doi.org/10.1016/S0953-7562 (09)80409-7

Richter C, Wittstein K, Kirk MP, Stadler M. 2015 - An assessment of the taxonomy and chemotaxonomy of Ganoderma. Fungal Diversity 71(1), 1-15. doi.org/10.1007/s13225-0140313-6

Ronquist F, Teslenko M, van der Mark P, Ayres DL et al. 2012 - MrBayes 3.2: Efficient Bayesian phylogenetic inference and model choice across a large model space. Systematic Biology 61(3), 539-542. doi.org/10.1093/sysbio/sys029

Ryvarden L. 2004 - Neotropical polypores Part 1. Synopsis Fungorum 19, 1-229. 
Salerni E, Laganá A, Perini C, Loppi S et al. 2002 - Effects of temperature and rainfall on fruiting of macrofungi in oak forests of the Mediterranean area. Israel Journal of Plant Sciences 50, 189-198. doi: 10.1560/GV8J-VPKL-UV98-WVU1

Sankaran KV, Bridge PD, Gokulapalan C. 2005 - Ganoderma diseases of perennial crops in Indiaan overview. Mycopathologia 159(1), 143-152. doi:10.1007/s11046-004-4437-1

Sawada K. 1931 - Descriptive catalogue of the Formosan fungi V. - Report of the Department of Agriculture Government Research Institute of Formosa 51, 1-131.

Seo GS, Kirk PM. 2000 - Ganodermataceae: nomenclature and classification. In: Flood J, Bridge PD, Holderness M (eds). Ganoderma Diseases of Perennial Crops. CABI. 3-23.

Singh SK, Kamal S, Tiwari M, Rai RD et al. 2004 - Myco-ecological studies of natural morel bearing sites in Shivalik hills of Himachal Pradesh, India. Micologia Aplicada International $16,1-6$.

Singh RP, Verma RC, Arora RK, Mishra KK et al. 2007 - Medicinal mushrooms of Uttaranchal with reference to Ganoderma, Auricularia and Cordyceps sinensis.In: Mushroom Biology and Biotechnology, Rai RD, Singh SK, Yadav MC, Tewari RP (eds). Mushroom Society of India. 322-324.

Song J, Xing JH, Decock C, HE XL et al. 2016 - Molecular phylogeny and morphology reveal a new species of Amauroderma (Basidiomycota) from China. Phytotaxa 260, 47-56. doi.org/10.11646/phytotaxa.260.1.5.

Smith BJ, Sivasithamparam K. 2000 - Internal transcribed spacer ribosomal DNA sequence of five species of Ganoderma from Australia. Mycological Research 104, 943-951.

Smith BJ, Sivasithamparam K. 2003 - Morphological studies of Ganoderma (Ganodermataceae) from the Australian and Pacific regions. Australasian Systematic Botany 16, 487-503 doi: 10.1071/SB02001.

Stamatakis A. 2014 - RAxML version 8: a tool for phylogenetic analysis and post-analysis of large phylogenies. Bioinformatics 30, 1312-1313. https://doi.org/10.1093/bioinformatics/btu0 33.

Straatsma G, Ayer F, Egli S. 2001 - Species richness, abundance, and phenology of fungal fruit bodies over 21 years in a Swiss forest plot. Mycological Research 105, 515-523. doi.org/10.1017/S0953756201004154

Suárez-Duque D. 2004 - Diversidad y análisis structural de los Aphyllophorales del Bosque Protector "Mindo Lindi", Prov. de Pichincha, Ecuador. Lyona 7, 83-89. doi.org/10.7550/rmb.27700

Swofford DL. 2002 - PAUP*. Phylogenetic analysis using parsimony (*and other methods) Version 4.0b10.Sinauer Associates, Sunderland. 10.1111/j.0014-3820.2002.tb00191.x

Szedlay G. 2002 - is the widely used medicinal fungus the Ganoderma lucidum (fr.) karst. sensu stricto? Acta Microbiologica et Immunologica Hungarica 49, 235-243. doi:10.1556/AMicr.49.2002.2-3.9

Teng SC.1934 - Notes on Polyporaceae from China. Sinensia 5, 198-200.

Teng SC. 1939. A contribution to our knowledge of the higher fungi of China. National Institute of Zoology \& Botany, Academia Sinica.

Teng SC. 1963 - Fungi of China. Beijing: Science Press, 808. (in Chinese).

Teng SC. 1964 - Fungi of China. Academia Sinica, Beijing (in Chinese).

Thunberg CP. 1784 - Flora Japonica, sistens Plantas insularum Japonicarum secundum systema sexuale emendatum redactas. - Mulleriano, J. G., Lipsiae, 418.

Vilgalys R, Hester M. 1990 -Rapid genetic identification and mapping of enzymatically amplified ribosomal DNA from several Cryptococcus species. Journal of Bacteriology 172, 4238-4246. doi.org/10.1128/jb.172.8.4238-4246.1990

Wang DM, Yao YJ. 2005 - Intrastrain internal transcribed spacer heterogeneity in Ganoderma species. Canadian journal of microbiology 51(2), 113-121. doi.org/10.1139/w04-118

Wang DM, Zhang XQ, Yao YJ. 2005 - Type studies of some Ganoderma species from China. Mycotaxon 93, 61-70. 
Wang DM, Wu SH, Su CH, Peng JT et al. 2009 - Ganoderma multipileum, the correct name for "G. lucidum" in tropical Asia. Botanical Studies 50, 451-458.

Wang DM, Wu SH. 2007 - Two species of Ganoderma new to Taiwan. Mycotaxon 102, 373-378.

Wang DM, Wu SH, Yao YJ. 2014 - Clarification of the concept of Ganoderma orbiforme with high morphological plasticity. PLoS ONE 9: e98733. doi.org/10.1371/journal.pone.0098733

Wu XL, Dai YC 2005 - Coloured illustrations of' Ganodermataceae' of China. Science Press.

White TJ, Bruns T, Lee S, Taylor J. 1990 - Amplification and direct sequencing of fungal ribosomal RNA genes for phylogenetics. In: Innis, MA, Gelfand DH, Sninsky JJ, White T.J (eds) PCR protocols: a guide to methods and applications. San Diego.

Xing J, Song J, Decock C, Cui B. 2016 - Morphological characters and phylogenetic analysis reveal a new species within the Ganoderma lucidum complex from South Africa. Phytotaxa 266, 115-124. doi.org/10.11646/phytotaxa.266.2.5

Xing JH, Sun YF, Han YL, Cui BK et al. 2018 - Morphological and molecular identification of two new Ganoderma species on Casuarina equisetifolia from China. MycoKeys 34, 93-108. doi.org/10.3897/mycokeys.34.22593

Yao YJ, Wang XC, Wang B. 2013 - Epitypification of Ganoderma sichuanense J. D. Zhao and X. Q. Zhang (Ganodermataceae). Taxon 62, 1025-1031. doi.org/10.12705/625.10.

Yang LZ, Feng B. 2013 - What is the Chinese "Lingzhi"? - A taxonomic mini-review. Mycology. An International Journal on Fungal Biology 4, 1-4. doi: 10.1080/21501203.2013.774299

Zhang X, Xu Z, Pei H, Chen Z et al. 2017 - Intraspecific variation and phylogenetic relationships are revealed by ITS1 secondary structure analysis and single-nucleotide polymorphism in Ganoderma lucidum. PLoS ONE 12(1): e0169042. doi:10.1371/journal. pone.0169042

Zhao JD, Hsu LW, Zhang XQ. 1979 - Taxonomic studies on the subfamily Ganodermoideae of China (in Chinese). Acta Mycologica Sinica 19, 265-279.

Zhao JD. 1989 - The Ganodermataceae in China. Bibliotheca Mycologica 132. Berlin: J. Cramer. 176.

Zhao JD, Zhang XQ. 2000 - Flora Fungorum Sinicorum 18: Ganodermataceae. Beijing: Science Press, 204. (in Chinese).

Zheng LY, Jia DH, Fei XF, Luo X et al. 2009 - An assessment of the genetic diversity within Ganoderma strains with AFLP and ITS PCR-RFLP. Microbiological Research 164(3), 312 321. doi: 10.1055/s-2008-1034289.

Zhou XW, Li QZ, Yin YZ, Chen YY et al. 2008 - Identification of medicinal Ganoderma species based on PCR with specific primers and PCR-RFLP. Planta Medica 74, 197-200. doi: 10.1055/s-2008-1034289.

Zhou LW, Cao Y, Wu SH, Vlasák J et al. 2015 - Global diversity of the Ganoderma lucidum complex (Ganodermataceae, Polyporales) inferred from morphology and multilocus phylogeny. Phytochemistry 114, 7-15. doi: 10.1016/j.phytochem.2014.09.023 